NASA Technical Memorandum 106986

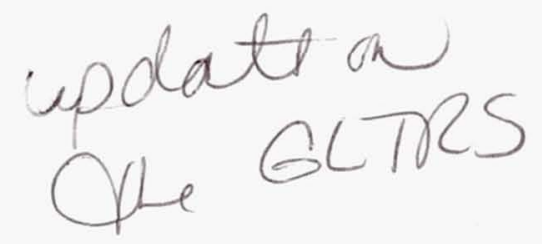

\title{
Modeling the Role of Dislocation Substructure During Class M and Exponential Creep
}

S.V. Raj

Lewis Research Center

Cleveland, Ohio

Ilana Seiden Iskovitz

Ohio Aerospace Institute

Cleveland, Ohio

and

\section{A.D. Freed}

Lewis Research Center

Cleveland, Ohio

August 1995

National Aeronautics and

Space Administration 
Also published in "Unified Constitutive Laws of Plastic Deformation," editors A.S. Krausz and K. Krausz, pp. 343-439, Academic Press, San Diego, 1996. 


\title{
MODELING THE ROLE OF DISLOCATION SUBSTRUCTURE DURING CLASS M AND EXPONENTIAL CREEP
}

\author{
S.V. Raj \\ National Aeronautics and Space Administration \\ Lewis Research Center \\ Cleveland, Ohio 44135 \\ Ilana Seiden Iskovitz \\ Ohio Aerospace Insitute \\ 22800 Cedar Point Road \\ Cleveland, Ohio 44142 \\ and \\ A.D. Freed \\ National Aeronautics and Space Administration \\ Lewis Research Center \\ Cleveland, Ohio 44135
}

\begin{abstract}
SUMMARY
The different substructures that form in the power-law and exponential creep regimes for single phase crystalline materials under various conditions of stress, temperature and strain are reviewed. The microstructure is correlated both qualitatively and quantitatively with power-law and exponential creep as well as with steady state and non-steady state deformation behavior. These observations suggest that creep is influenced by a complex interaction between several elements of the microstructure, such as dislocations, cells and subgrains. The stability of the creep substructure is examined in both of these creep regimes during stress and temperature change experiments. These observations are rationalized on the basis of a phenomenological model, where normal primary creep is interpreted as a series of constant structure exponential creep rate-stress relationships. The implications of this viewpoint on the magnitude of the stress exponent and steady-state behavior are discussed. A theory is developed to predict the macroscopic creep behavior of a single phase material using quantitative microstructural data. In this technique the thermally activated deformation mechanisms proposed by dislocation physics are interlinked with a previously developed multiphase, three-dimensional, dislocation substructure creep model. This procedure leads to several coupled differential equations interrelating macroscopic creep plasticity with microstructural evolution.
\end{abstract}




\section{LIST OF SYMBOLS}

A dimensionless constant in the power-law equation (3)

$\mathrm{A}_{\text {H.T. }}$ dimensionless constant in the high temperature climb equation (5)

A $_{\text {L.T. }}$ dimensionless constant in the low temperature climb equation (6)

$\mathrm{A}_{1} \quad$ constant in the exponential creep equation (4)

$\mathrm{A}_{2} \quad$ constant in equation $(22 \mathrm{~b})$

A activation area for a thermally activated deformation process

a lattice parameter

B dimensionless constant in the exponential creep equation (4)

$\mathrm{B}_{2} \quad$ constant in equation (22b)

$\mathrm{B}_{\mathrm{ij}} \quad$ tensorial representation of the back stress

$\vec{b} \quad$ Burgers vector

$\mathrm{C}_{\mathrm{j}} \quad$ density of extended jogs

$\mathrm{D}_{\text {eff }} \quad$ effective diffusion coefficient for lattice and pipe diffusion

$\mathrm{D}_{\mathrm{ijkl}} \quad$ elastic moduli tensor

$\mathrm{D}_{1} \quad$ lattice self-diffusion coefficient

$\mathrm{D}_{0} \quad$ frequency factor for diffusion

$\mathrm{D}_{01} \quad$ frequency factor for lattice diffusion

$\mathrm{D}_{0 \mathrm{p}} \quad$ frequency factor for pipe diffusion

d grain size

$\mathrm{d}_{\mathrm{c}} \quad$ cell size

$\mathrm{d}_{\text {cs }} \quad$ stress dependent stacking fault width

$\mathrm{d}_{\mathrm{s}} \quad$ subgrain size

$d_{s b} \quad$ diameter of new subgrains nucleated by localized migration of a pre-existing subboundary $\left(=\beta_{1} \Gamma_{s b} / \tau\right)$

$\overline{\mathrm{E}}_{\mathrm{ij}} \quad$ applied or global strain tensor

$\mathrm{E}_{\mathrm{ij}}^{\phi} \quad$ local deviatoric strain tensor in phase $\phi$

$\dot{\mathrm{E}}_{\mathrm{ij}}^{\phi} \quad$ local deviatoric strain rate tensor in phase $\phi$ 
$\mathrm{F}_{\mathrm{ij}} \quad$ tensorial representation of the forward stress

$\Delta \mathrm{F} \quad$ Helmholtz free energy associated with moving a dislocation past an obstacle in equation (11)

$f^{c b} \quad$ volume fraction of cell boundaries

$\mathrm{f}^{\text {h }} \quad$ volume fraction of 'hard' regions

$\mathrm{f}^{\mathrm{s}} \quad$ volume fraction of 'soft' regions

G shear modulus

$\Delta \mathrm{G}_{\mathrm{CS}} \quad$ activation free energy for cross-slip at zero stress in equation (10)

h dislocation spacing in a cell or subboundary

$\mathrm{I}_{\mathrm{k} \mathrm{mn}} \quad$ identity tensor

$\mathrm{K} \quad$ constant relating the subgrain size and the applied stress in equation (16)

$\mathrm{K}_{1} \quad$ constant relating dislocation spacing in the cell or subgrain boundaries with the stress in the 'hard' regions

k Boltzmann's constant $\left(1.38 \times 10^{-23} \mathrm{~J} \mathrm{~K}^{-1}\right)$

L general representation of the cell $\left(d_{c}\right)$ or subgrain $\left(d_{s}\right)$ size

$\mathrm{L}^{\mathrm{h}} \quad$ average dimensions of the 'hard' regions

$\mathrm{L}_{\mathrm{N}} \quad$ dislocation spacing in a network

$\mathrm{L}^{\mathrm{S}} \quad$ average dimensions of the 'soft' regions

L* activated length for cross-slip

M Taylor factor

m subgrain size exponent in equation (16)

$\mathrm{N} \quad$ number of cell or subboundaries

$N_{P} \quad$ number of dislocations in a piled-up array in equation (8)

n creep stress exponent

n' effective creep stress exponent

$\mathrm{Pj} \quad$ jth material property

$\mathrm{p} \quad$ constant in equation (4) obtained from the stress dependence of the dislocation density. In general, $\mathrm{p} \approx 2$ or 3 for most materials

$Q_{C} \quad$ activation energy for creep 
$\mathrm{Q}_{\mathrm{cs}} \quad$ activation energy for cross-slip mechanisms

$\mathrm{Q}_{\mathrm{g}} \quad$ activation energy for the obstacle-controlled glide process

$Q_{1} \quad$ activation energy for lattice self-diffusion

$\mathrm{Q}_{0} \quad$ maximum activation energy for cross-slip in equation (9b)

$\mathrm{Q}_{01} \quad$ maximum activation energy for cross-slip in equation (10)

$Q_{p} \quad$ activation energy for dislocation core diffusion

$\mathrm{Q}_{\mathrm{Cl}^{-}}$activation energy for $\mathrm{Cl}^{-}$diffusion in $\mathrm{NaCl}$

$\mathrm{Q}_{\mathrm{Na}}{ }^{+}$activation energy for $\mathrm{Na}^{+}$diffusion in $\mathrm{NaCl}$

q $\quad$ stacking fault energy exponent

$\mathrm{R} \quad$ universal gas constant $\left(8.314 \mathrm{~J} \mathrm{~mol}^{-1} \mathrm{~K}^{-1}\right)$

$\mathrm{S}_{\mathrm{i}} \quad$ microstructural parameters $1,2,3 \ldots \mathrm{i}$

$\mathrm{S}_{\mathrm{ij}}^{\phi} \quad$ deviatoric stress in phase $\phi$

$\dot{\mathrm{S}}_{\mathrm{ij}}^{\phi} \quad$ deviatoric stress rate tensor in phase $\phi$

$\mathrm{S}_{\mathrm{ijkl}} \quad$ Eshelby deviatroic stress tensor

T absolute temperature

$\mathrm{T}_{\mathrm{c}} \quad$ absolute transition temperature above which $\mathrm{Q}_{\mathrm{c}} \approx \mathrm{Q}_{1}$ and below which $\mathrm{Q}_{\mathrm{c}}<\mathrm{Q}_{1}$

$\mathrm{T}_{\mathrm{m}} \quad$ absolute melting temperature

$\mathrm{T}_{0} \quad$ reference temperature in equation $(57)$

t time

$t_{c}$ characteristic time for a pair of edge dislocations to climb a distance $\mathrm{h} / 2$ before annihilation

$\mathrm{V}^{*} \quad$ activation volume for creep

$v_{c} \quad$ climb velocity

$\mathrm{X}_{\mathrm{ij}}^{\phi} \quad$ general representation of a local tensorial strain or stress field in phase $\phi$

$\bar{X}_{\mathrm{ij}} \quad$ global tensorial strain or stress field

x spacial coordinate

$\alpha \quad$ geometric constant in the Taylor equation (20)

$\alpha_{c} \quad$ coefficient of thermal expansion 


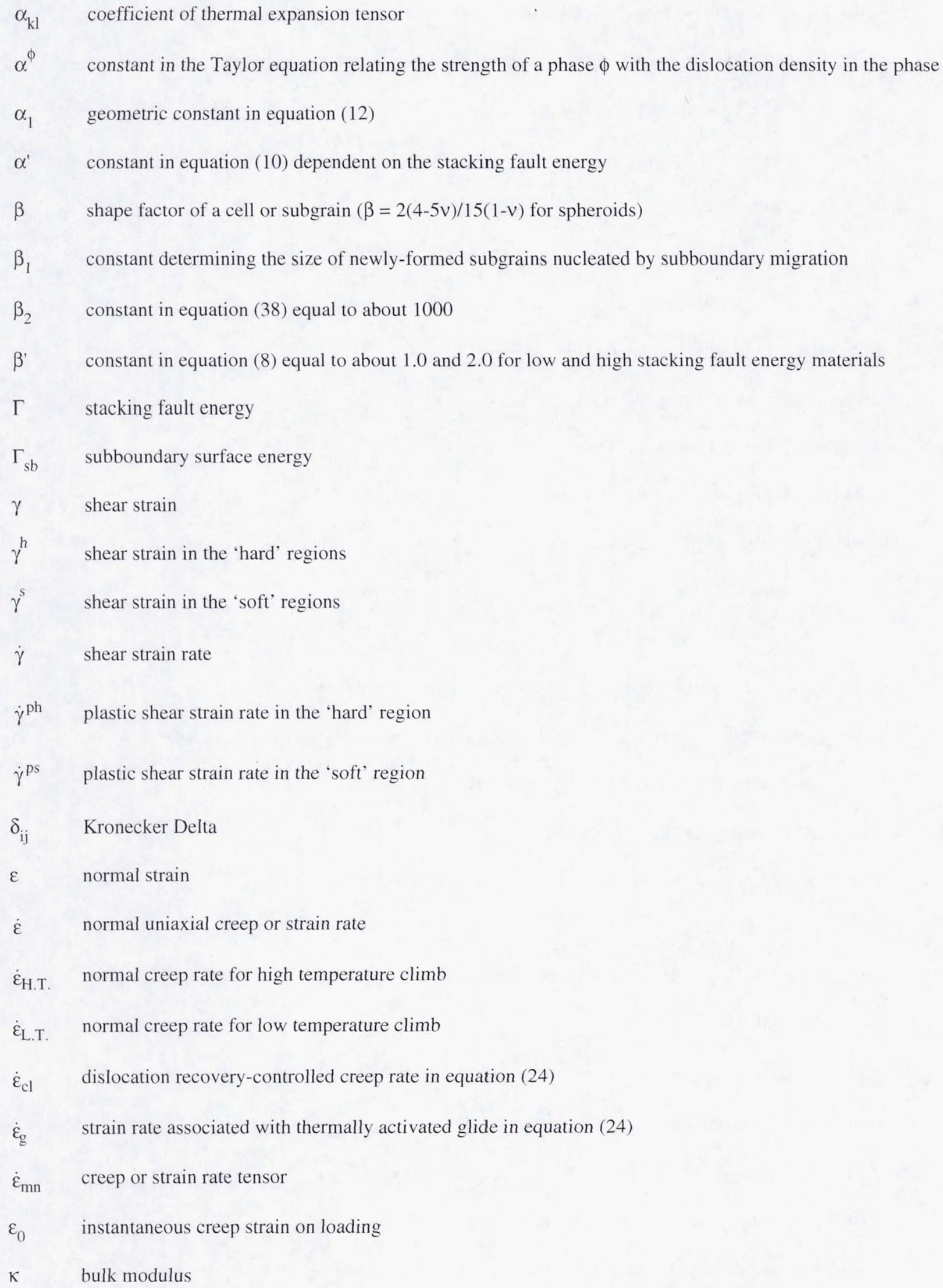




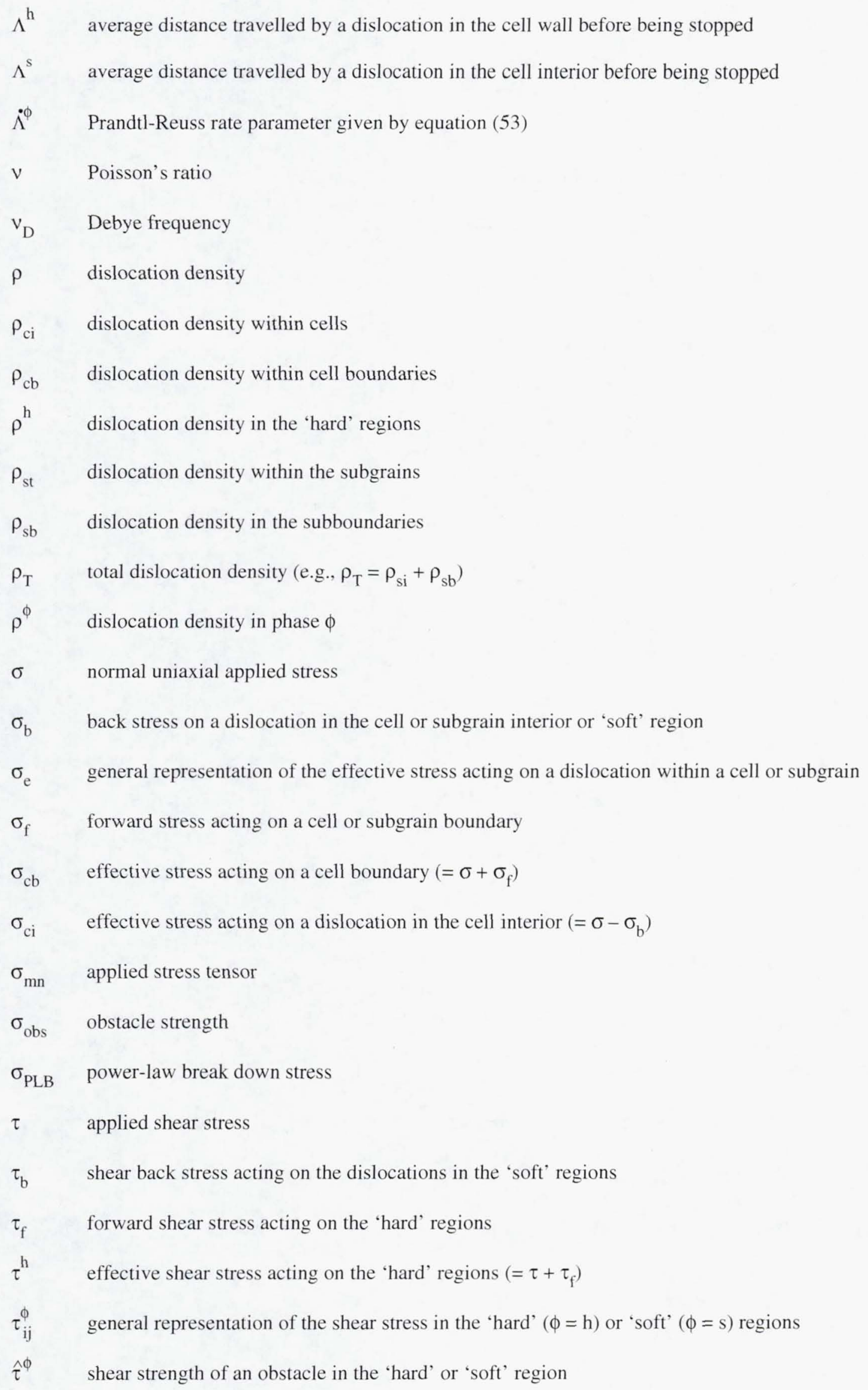


$\tau$ s effective shear stress acting in the 'soft' regions $\left(=\tau-\tau_{\mathrm{b}}\right)$

$\phi \quad$ general representation denoting 'hard' (h) or 'soft' (s) phase

$\chi \quad=\mathrm{L}^{\mathrm{h}} / \mathrm{L}^{\mathrm{s}}$

$\chi_{0} \quad$ initial value of $\chi$ at the beginning of deformation

$\Omega \quad$ atomic volume 


\subsection{INTRODUCTION}

\subsection{Kinetic and Microstructure Evolution Laws}

Creep is defined as the time-dependent deformation of a material at any absolute temperature, $\mathrm{T}$, under the action of either a slowly time variant or a time invariant monotonic applied stress. Although a material can creep at any temperature above absolute zero, measurable time-dependent deformation occurs at elevated temperatures typically above $0.3 \mathrm{~T}_{\mathrm{m}}$, where $\mathrm{T}_{\mathrm{m}}$ is the absolute melting temperature of the material. Considerable data, accumulated over several decades, have enabled a detailed characterization of the creep process in terms of its stress, temperature and microstructural dependence. As a result, several models have attempted to formulate constitutive laws to express the creep rate, $\dot{\varepsilon}$, in terms of $\sigma$ or $\tau, \mathrm{T}, \mathrm{P}_{\mathrm{j}}$ and $\mathrm{S}_{\mathrm{i}}$, where $\sigma$ and $\tau$ are the applied normal and shear stress, respectively, $\mathrm{P}_{\mathrm{j}}$ is the $\mathrm{j}^{\text {th }}$ material property (e.g. lattice parameter, a, atomic volume, $\Omega$, elastic moduli, Burgers vector, $b$, and diffusion frequency factor, $D_{0}$ ) and $S_{i}$ is the $i^{\text {th }}$ microstructural parameter (e.g., grain size, d, dislocation density, $\rho$, cell size, $d_{c}$, subgrain size, $d_{s}$, and stacking fault energy, $\Gamma$ ). It should be noted that $S_{i}$ is also referred to as an internal state variable in the plasticity literature. A more general formulation can express the tensorial creep rate, $\dot{\varepsilon}_{\text {mn }}$, in terms of a tensorial stress state, $\sigma_{\mathrm{mn}}$. The role of microstructure in influencing creep behavior has been studied extensively in a large number of materials as evidenced by the numerous review articles (Schoeck, 1957; Sherby, 1962; McLean, 1966; Sherby and Burke, 1967; Bird et al., 1969, Mukherjee et al., 1969; Lagneborg, 1972; Weertman, 1975; Takeuchi and Argon, 1976; Blum, 1977; Nix and Ilschner, 1980; Myshlyaev, 1981; Bendersky et al., 1985; Nix and Gibeling, 1985(a); Orlová and Cadek, 1986; Caillard and Martin, 1987; Blum et al., 1991; Blum, 1993; Longquan and Northwood, 1993; Yoshinaga, 1993; Raj, 1994) and texts (Garofalo, 1965; Gittus, 1975; Poirier, 1985) which have followed the course of development of the subject over the last four decades. These advances suggest that deformation behavior can be represented by a generalized kinetic or rate equation (Frost and Ashby, 1982)

$$
\dot{\varepsilon}=f\left(\sigma, T, S_{i}, P_{j}\right)
$$

and a generalized microstructure evolution equation describing the rate of change of the microstructure, $\dot{S}_{i}$, during deformation

$$
\dot{\mathrm{S}}_{\mathrm{i}}=\mathrm{g}\left(\sigma, \mathrm{T}, \mathrm{S}_{\mathrm{i}}, \mathrm{P}_{\mathrm{j}}\right)
$$

It is important to note that the kinetic equation (1) describes deformation for a constant microstructure, whereas the microstructure evolution equation (2) represents the manner in which the microstructure changes during the course of deformation. Equations (1) and (2) are fairly general to be applicable to both low and high temperature deformation under either cyclic or monotonic stresses. This generality argues for the development of a unified deformation model which describes flow behavior under different stress states at any temperature for conditions where thermally activated dislocation processes are important. In reality, the problem is complex in part because the present understanding of the dislocation mechanisms controlling deformation is incomplete in many instances and in part because the role of microstructure and its evolution on deformation processes is difficult to quantify. Thus, specific deformation characteristics and microstructure may vary from one material to another. Nevertheless, the last four decades of research have shown that there are a lot of similarities in the deformation behavior and microstructural evolution in several materials ranging from simple metals to the more complex geological rocks (Frost and Ashby, 1982). These similarities have permitted a general understanding of the deformation behavior of materials.

The emphasis of the present chapter is to consider dislocation-controlled flow processes, except HarperDorn creep (Harper and Dorn, 1957; Harper et al., 1958), occurring under uniaxial deformation. The mechanism for Harper-Dorn creep is poorly understood at present and this process is not considered. In contrast to classical approaches, which artificially treat low and high temperature deformation as unrelated flow behavior, the current treatment views deformation from a broader global perspective of a material response to a set of external variables resulting from a corresponding evolution in the microstructure. Dislocations within a material move, rearrange and annihilate themselves and form low energy dislocation substructures (LEDS) so as to achieve steady-state conditions 
in accordance with the laws of irreversible thermodynamics. In principle, this steady-state condition and the corresponding microstructure is achievable under all deformation conditions at all temperatures above $0 \mathrm{~K}$ if other factors, such as mechanical and microstructural instability, do not intervene. However, in practice the rate of recovery may be extremely slow so that steady-state deformation conditions and an equilibrium microstructure may not be observed in the time frame of a typical experiment.

\subsection{Types of Flow Behavior}

Figure 1 shows schematics of the different types of tensile creep curves, plotted either as creep strain, $\varepsilon$, against time, t, (A) or as $\dot{\varepsilon}$ versus $\varepsilon$ (B), generally observed in creep tests. The corresponding tensile $\sigma-\varepsilon$ curves (C) obtained from constant strain rate tests are also shown since the latter tests are often used for generating steady-state $\dot{\varepsilon}-\sigma$ plots in studies relating to the high temperature deformation of materials. Some special cases, such as serrated yielding and dynamic recrystallization, are not discussed. Although the constant strain rate and creep data often correspond closely at high temperatures, it is important to recognize that the transient dislocation substructures formed in these two tests are likely to be vastly different. This difference arises from the fact that the thermodynamic path by which the substructure evolves towards a steady-state configuration will be generally different in constant stress and constant strain rate tests.
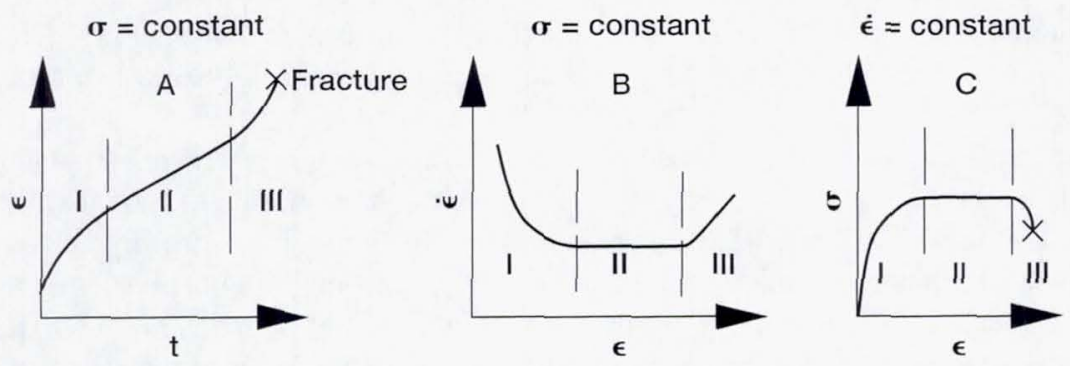

(a)
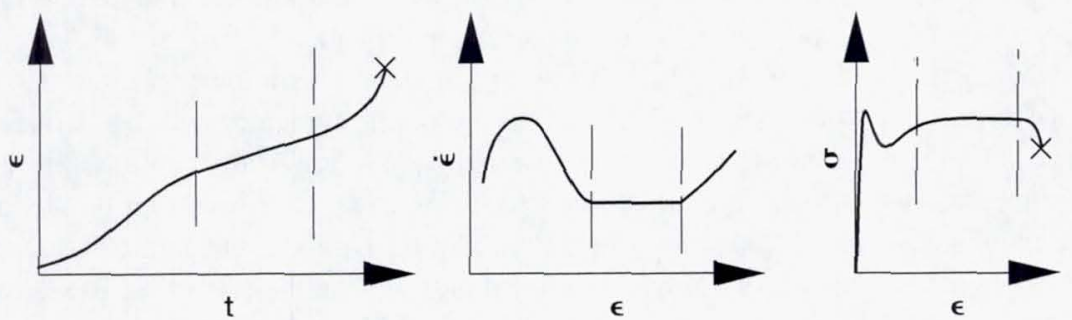

(b)
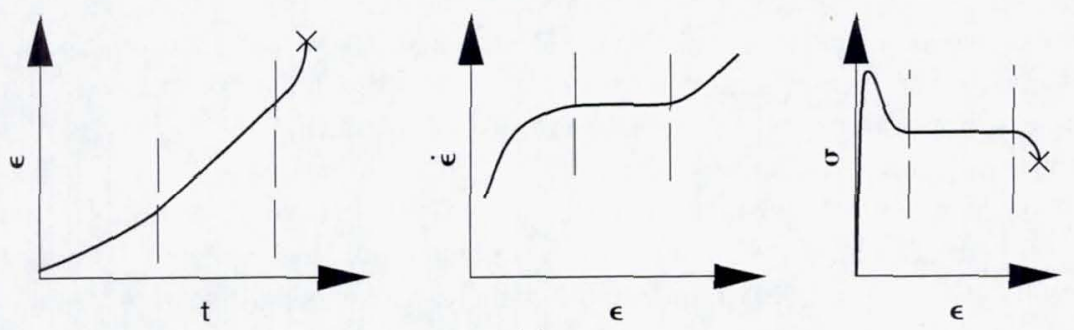

(c)

Figure 1.-Schematic plots of creep strain versus time (A), creep rate versus creep strain (B) and stress versus strain (C) illustrating typical (a) normal, (b) sigmoidal and (c) inverse primary creep and the corresponding stressstrain transients observed under constant stress and constant strain rate deformation conditions. Regions I, II, and III represent the primary, secondary and tertiary regions. 
A typical creep curve consists of several stages (Fig.1): an instantaneous strain on loading; a primary creep region where the creep rate changes with time (I); a region of linear, steady-state flow or secondary creep regime in which $\dot{\varepsilon}$ is independent of strain and time (II); and a period of accelerating creep in the tertiary stage resulting from mech-anical (i.e. external or internal necking) or microstructural (e.g. dynamic recrystallization and grain growth) instability (III). The duration of secondary creep will be short if tertiary creep occurs early. Tertiary creep is generally absent in compression creep if microstructural instability does not occur. However, the creep rate can continue to decrease with strain or time during constant load compression creep due to an increase in the cross-sectional area.

Normal primary creep, typical of class M or Metal type (Yavari et al., 1981) or class II (Sherby and Burke, 1967) behavior, is illustrated by Fig. 1(a), where the creep rate decreases with increasing strain in the primary region as the material work hardens. In the absence of significant recovery, the material will continue to harden until the creep rate is immeasurably small. However, significant recovery occurs in most materials at intermediate and high temperatures, ${ }^{1}$ and the material tends towards steady-state behavior where the work hardening rate balances the recovery rate. Under these conditions, all microstructural parameters attain quasi-equilibrium so that $\mathrm{dS}_{\mathrm{i}} / \mathrm{dt} \approx 0$. Sigmoidal (Fig. 1(b)) and inverse (Fig. 1(c)) primary creep are often observed in materials exhibiting class A or Alloy type (Yavari et al. 1981) or class I (Sherby and Burke, 1967) behavior, where a solute atmosphere around a dislocation tends to inhibit its motion (Weertman, 1957). In some instances, steady-state flow is attained almost immediately on loading and the creep curve is linear until tertiary creep intervenes. Inverse and sigmoidal primary creep occur when the initial mobile dislocation density is low which results in a low initial creep rate. The creep rate increases with strain during inverse primary creep until a steady-state value is obtained (Fig. 1(c)). Sigmoidal primary creep, which is intermediate between inverse and normal primary creep, involves an initial region of increasing creep rate to a maximum value followed by a period of decreasing creep rate till steady-state creep is achieved (Fig. 1(b)).

Most creep mechanisms fall into three broad categories: diffusion, power-law and exponential creep. Two diffusion creep mechanisms involving Newtonian viscous flow with $\dot{\varepsilon}$ proportional to $\sigma$ (Nabarro, 1948; Herring, 1950; Coble, 1963) occur at low values of normalized stress, $\sigma / G$ with $G$ being the shear modulus, where typically $\sigma / \mathrm{G} \leq 5 \times 10^{-6}$. In this case, strain is produced by the diffusion of atoms from grain boundaries under compression to those under tension with vacancies flowing in the opposite direction (Raj and Ashby, 1971). Nabarro-Herring creep (Nabarro,1948; Herring, 1950) occurs when the diffusion path is through the lattice in contrast to Coble creep (Coble, 1963), where the diffusion path is along grain boundaries. A third Newtonian creep mechanism, which was discovered by Harper and Dorn $(1957,1958)$, involves a poorly-understood dislocation-controlled process.

In contrast, power-law creep occurs at intermediate stresses, typically $5 \times 10^{-6} \leq \sigma / \mathrm{G} \leq \sigma_{\mathrm{PLB}} / \mathrm{G}$, where $\sigma_{\mathrm{PLB}}$ is the power-law breakdown (PLB) stress. In this case, the creep rate depends on the applied stress through a powerlaw relation with $\dot{\varepsilon}$ proportional to $(\sigma / \mathrm{G})^{\mathrm{n}}$, where $\mathrm{n}$ is the stress exponent, typically varying between 4 and 5 for class $\mathrm{M}$ behavior (Bird et al., 1969). Strain is produced by a sequential motion of dislocations through glide and climb, where the slowest mechanism is the rate-controlling step. Since the power-law relation is a generic equation which can often be used to describe almost any set of creep data plotted double logarithmically, the term "power-law creep" is not very descriptive of the characteristics of the deformation mechanism. Instead, the terms "class M creep" and "class A creep" will be used in this paper to describe class M or class A material behavior, respectively.

The power-law relation breaks down at high stresses when $\sigma / \mathrm{G}>\sigma_{\mathrm{PLB}} / \mathrm{G}$ and the stress dependence of $\dot{\varepsilon}$ is better described by an exponential relationship with $\dot{\varepsilon}$ proportional to $\exp (\mathrm{B} \sigma / \mathrm{G})$, where $\mathrm{B}$ is a constant. Unlike creep mechanisms dominant in the diffusion and class $\mathrm{M}$ creep regimes, where the diffusion of atomic and defect species is important, exponential creep mechanisms are generally non-diffusional in nature. Since the major objective of the present chapter is to model the role of dislocation substructure during class $\mathrm{M}$ and exponential creep, class A and Newtonian viscous creep mechanisms are not addressed in this review.

In contrast to class $\mathrm{M}$ creep, which has been studied extensively in several materials (Sherby and Burke, 1967; Bird et al., 1969; Mukherjee et al., 1969), considerably less attention has been paid to understanding the mechanisms dominant in the exponential creep region and the microstructures that form under these conditions. Noting that many engineering applications require materials to operate at intermediate temperatures (Table I) (Nix and Gibeling, 1985(a)), where the diffusion rates are slower than at higher temperatures, the rate-controlling mechanisms may often be nondiffusional in nature and similar to those dominant in the exponential creep region (Raj and

\footnotetext{
'The terms 'intermediate' and 'high' as used in the creep literature strictly refer to the temperature ranges above $0.3 \mathrm{~T}_{\mathrm{m}}$ for which $\mathrm{Q}_{\mathrm{c}}<\mathrm{Q}_{1}$ and $Q_{C} \approx Q_{1}$, respectively, where $Q_{C}$ and $Q_{1}$ are the activation energies for creep and lattice self diffusion, respectively. For simplicity, the temperature ranges $T \approx 0.3-0.7 \mathrm{~T}_{\mathrm{m}}$ and $\mathrm{T}>0.7 \mathrm{~T}_{\mathrm{m}}$ are generally assumed to define the intermediate and high temperature creep ranges, respectively, since $\mathrm{Q}_{\mathrm{c}} \approx \mathrm{Q}_{1}$ when $\mathrm{T} \geq 0.7 \mathrm{~T}_{\mathrm{m}}$ for most materials.
} 
TABLE I.-TYPICAL OPERATING TEMPERATURES IN

ENGINEERING APPLICATIONS.

(Nix and Gibeling, 1985(a)).

\begin{tabular}{|l|l|c|c|}
\hline \multicolumn{1}{|c|}{ Application } & Typical materials & $\begin{array}{c}\text { Typical } \\
\text { temperatures, } \\
\mathrm{K}\end{array}$ & T/T $\mathrm{m}$ \\
\hline $\begin{array}{l}\text { Rotors and piping } \\
\text { for steam turbines }\end{array}$ & Cr-Mo-V steels & 825 to 875 & 0.45 to 0.50 \\
$\begin{array}{l}\text { Pressure vessels } \\
\text { and piping in } \\
\text { nuclear reactors }\end{array}$ & 316 stainless steel & 650 to 750 & 0.35 to 0.40 \\
$\begin{array}{l}\text { Reactor skirts in } \\
\text { nuclear reactors }\end{array}$ & 316 stainless steel & 850 to 950 & 0.45 to 0.55 \\
$\begin{array}{l}\text { Gas turbine blades } \\
\text { Burner cans for } \\
\text { gas turbine engines }\end{array}$ & $\begin{array}{l}\text { Nickel-based } \\
\text { superalloys } \\
\text { Oxide dispersion } \\
\text { strengthened } \\
\text { nickel-based alloys }\end{array}$ & 1350 to 1400 & 0.55 to 0.65 \\
\hline
\end{tabular}

Langdon, 1989; 1991(a) and (b)). Despite the potential importance of characterizing exponential creep behavior, this subject has been largely ignored primarily because of the difficulty in modeling nonsteady state creep. Since creep data are seldom acquired over a very large range of stresses and temperatures in any one investigation, detailed microstructural characterization of the transition from class $M$ to exponential creep is virtually nonexistant for most materials. As a result, our understanding of the microstructural changes occurring during this transition is poor so that it is difficult to formulate realistic microstructurally-based creep models.

Nevertheless, in comparison to previous attempts (Weertman, 1955, 1957, 1975; McLean, 1966; Lagneborg, 1972; Takeuchi and Argon, 1976), a fundamental change occurred in the philosophy and approach adopted towards the development of microstructure-based creep models when Nix and Ilschner (1980) developed a two-phase microstructural composite creep model to predict deformation behavior in the class $\mathrm{M}$ and exponential creep regimes. It is interesting to note that Mughrabi (1980) and Mughrabi and Essmann (1980) also presented a similar approach in modeling low temperature behavior and fatigue at the same conference. Although the NixIlschner model (1980) is conceptually incorrect (Raj and Langdon, 1991(b)), it provided the foundation for the development of current creep models. A significant step in this direction occurred with the publication of two important papers by Nix et al., (1985(a) and (b)), which attempted to model creep using a set of coupled kinetic and evolution equations describing low and high temperature deformation mechanisms. Other developments and refinements followed (Vogler and Blum, 1990; Hofmann and Blum, 1993; Zhu and Blum, 1993). Typically, these models assume that the microstructure consists of alternate regions of long subboundaries or 'hard' phases and subgrain interiors or 'soft' phases, which must deform in such a manner so as to maintain compatibility between the two phases. When considered with similar modeling efforts for low temperature uniaxial (Mughrabi, 1983; Argon and Haasen, 1993) and cyclic (Mughrabi, 1981, 1987) deformation, it becomes apparent that, at least in principle, this approach to modeling low and high temperature deformation behavior can lead to the development of a unified model describing various deformation behavior of a material.

Despite the potential advantages of the two-phase deformation models, they are still largely limited in scope for several reasons. First, they assume that subgrains form soon after loading, and therefore, they do not take into account the large microstructural variations that actually occur during primary creep and in the exponential creep region. Second, they unrealistically assume that subgrains are one-dimensional in nature. Third, they assume that the volume fractions of the subboundaries and dislocation densities do not change with strain or time. Fourth, they assume the presence of one type of substructure i.e., cells or subgrains. In reality, as discussed in $\$ 3.1 .2$, both cells and subgrains can form during creep.

In a recent development, Freed et al., (1992) attempted to address some of these issues by extending the one-dimensional composite models to the more realistic three dimensions using an Eshelby analysis for isotropic spheroidal cells or subgrains, and by allowing the microstructural terms to vary with deformation. The approach was 
general enough to consider a variant microstructure of cells and subgrains coexisting together although, for simplicity, the analysis was presented for one type of substructure. It was demonstrated that the shape factor, $\beta$, of the cell or subgrain introduces an additional second order tensorial elastic strain term to describe the local strain compatibility between the 'soft' and 'hard' regions in the crystal. The equations reduce to the Voigt approximation used by Nix and others in the one-dimensional case when $\beta=0$. In principle, this three-dimensional model, termed as Dislocation Substructure Viscoplasticity (DSV), should lead to the development of a unified deformation theory to describe plastic deformation for any stress state with additional refinements.

In contrast to the microstructural-based creep theories, viscoplasticity models ignore the role of microstructure on creep behavior (Kocks, 1987). Instead, the material is treated as a "black box" with stress and temperature as the primary input variables, and creep strain and creep rate as the main output variables. The internal state variables are introduced to account for transient effects, such as the Bauschinger effect and cyclic hardening under fatigue type loading situations. This approach is more useful in predicting the material response in engineering design, whereas the microstructural theories permit sound material design and selection. However, both approaches to creep modeling are limited in scope in that the viscoplastic models have to make arbitrary assumptions regarding $\mathrm{S}_{\mathrm{i}}$ while current microstructural-based creep theories cannot be easily scaled-up to predict the macroscopic creep behavior of an engineering component.

Therefore, the question arises: Can a model be developed by coupling the creep equations from viscoplasticity with those from dislocation physics? If so, then it should be possible, at least in theory, to predict the macroscopic creep response of an engineering component or test specimen at one extreme and the evolving deformation microstructure at the other using the principles of finite element analysis (FEM), viscoplasticity and dislocation physics. The fundamental objective of this chapter is to develop such a modeling technique. In its final form the model should predict the time evolution of the shape of a deforming body and the corresponding internal stresses and dislocation microstructures for macroscopic stress states produced during bend, cyclic, multiaxial and uniaxial tests. However, owing to the complexity of the problem and the amount of computational time required, the present goals are rather modest and tackle only uniaxial deformation. The intention here is to present a focussed review of uniaxial compression creep data and microstructural observations on $\mathrm{NaCl}$ single crystals with the primary purpose of using the information in developing the creep model. No attempt is made to specifically review the creep properties of other materials. The use of $\mathrm{NaCl}$ single crystal data in this study was influenced by a number of factors critical to the present modeling effort. Since it is a single phase material, which exhibits class $\mathrm{M}$ creep behavior, additional complications due to solid solution effects and second phase particles are avoided. In addition, grain boundary effects are eliminated for single crystals. More importantly, there is a considerable amount of creep, and qualitative and quantitative microstructural data on this material varying over a very large range of stresses and temperatures corresponding to class M and exponential creep (Ilschner and Reppich, 1963; Le Comte, 1965; Stokes, 1966; Blum and Ilschner, 1967; Heard, 1972; Poirier, 1972(a) and (b); Eggeler and Blum, 1981; Carter and Hansen, 1983; Wawersik, 1984, 1985; Raj and Pharr, 1986(a) and (b); Nadgornyi and Strunk, 1987; Przystupa et al., 1987; Raj et al., 1989; Raj and Pharr, 1989; Raj et al., 1991; Raj and Freed, 1992; Raj and Pharr, 1992).

Figure 2 schematically illustrates the approach adopted in this work. The essential idea used in developing this mesoscopic creep model is to couple the microscopic kinetic and evolution equations for different thermally activated deformation mechanisms with the macroscopic stresses, strains, strain rates and time-dependent microstructural variables using the DSV model (Freed et al., 1992). A solution of the equations from the DSV model at different points on an engineering component or a test specimen using FEM analysis then gives the macroscopic response in terms of stresses, strains and creep rates. This coupling between the macro- and the microstates of the component allows a two-way flow of information as shown in Fig. 2. In essence, this approach promises to be a powerful analytical tool for understanding the deformation behavior of solids under uniaxial, multiaxial and cyclic loading conditions if the details of the dislocation mechanisms are well characterized for the dominant deformation processes. However, this paper provides only the theoretical basis for coupling the DSV model and dislocation mechanics. The dislocation model considered here is applicable only to the simple one-dimensional cell model postulated by Nix et al., (1985)(a) and (b). A more detailed treatment of the dislocation processes governing cell and subgrain formation and their subsequent deformation is required before the DSV model can be applied to the general three-dimensional case. In addition, the extension of the computation to study the deformation of an entire test specimen using numerical analysis and FEM is not considered here in order to keep the problem tractable. For simplicity, grain boundary effects are neglected so that the present solutions are strictly valid for single crystals. The solutions are valid for polycrystalline materials only if the strain contributions from grain boundary effects, such as grain boundary sliding and grain boundary migration, are small. 


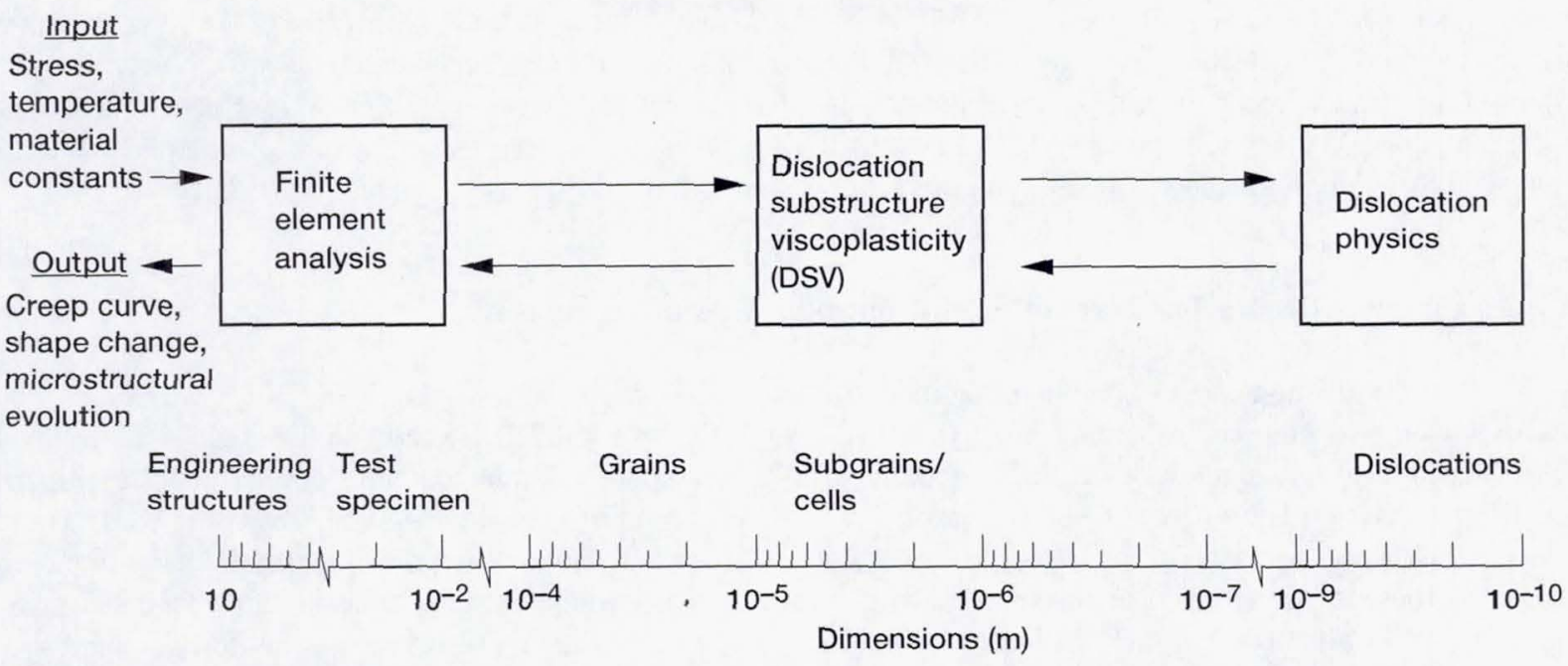

Figure 2.-Schematic block diagram of the coupling between dislocation mechanics, dislocation substructure viscoplasticity and finite element analysis required to study the interaction between the macroscopic and microscopic stresses, strains and strain rates.

This chapter is divided into several sections: First, a general discussion of the current understanding of the class $\mathrm{M}$ and exponential creep mechanisms is presented for single phase class $\mathrm{M}$ materials. Second, the characteristics of the dislocation substructures formed during class $M$ and exponential creep are discussed. Third, the stability of these microstructures after stress and temperature changes are examined. Fourth, a modified version of the onedimensional, two-phase Nix-Gibeling creep model (1985(a)) is presented. Fifth, the development of the DSV model is treated.

\subsection{CLASS M AND EXPONENTIAL CREEP IN SINGLE PHASE MATERIALS}

The steady-state creep rate at intermediate values of normalized stress corresponding to the class $\mathrm{M}$ creep regime is usually given by (Bird et al., 1969)

$$
\dot{\varepsilon}=\mathrm{A}\left(\mathrm{D}_{0} \mathrm{~Gb} / \mathrm{kT}\right)(\sigma / \mathrm{G})^{\mathrm{n}} \exp \left(-\mathrm{Q}_{\mathrm{c}} / \mathrm{RT}\right)
$$

where $\mathrm{b}$ is the Burgers vector, $\mathrm{k}$ is Boltzmann's constant, $\mathrm{R}$ is the universal gas constant, and $\mathrm{A}$ is a dimensionless constant. Typically, the experimental values of the stress exponents are about $n \approx 4$ to 5 and $Q_{c} \approx Q_{1}$ for a large number of materials exhibiting class M behavior (Sherby and Burke, 1967). A point that may be noted here is that the observation $Q_{c} \approx Q_{1}$ is not accepted by all investigators. For example, Poirier $(1978,1979)$ has suggested that $\mathrm{Q}_{\mathrm{c}}>\mathrm{Q}_{1}$ at very high temperatures and low stresses, and that $\mathrm{Q}_{\mathrm{c}} \approx \mathrm{Q}_{1}$ is only an approximation of true deformation behavior in certain stress and temperature ranges. This viewpoint has been questioned by Sherby and Weertman (1979) and Nix and Ilschner (1980). At high values of normalized stresses, the power-law relation given by equation (3) breaks down when $\sigma / \mathrm{G}>(\sigma / \mathrm{G})_{\mathrm{PLB}}$, where $(\sigma / \mathrm{G})_{\mathrm{PLB}}$ is the normalized power-law breakdown stress. The creep rate then exhibits an exponential dependence on normalized stress over a large range of stresses and temperatures as

$$
\dot{\varepsilon}=\mathrm{A}_{1}(\sigma / \mathrm{G})^{\mathrm{p}} \exp (\mathrm{B} \sigma / \mathrm{G}) \exp \left(-\mathrm{Q}_{\mathrm{c}} / \mathrm{RT}\right)
$$

where $\mathrm{A}_{1}$ and $\mathrm{p}$ are constants, where $\mathrm{p}$ reflects the stress dependence of the dislocation density so that $\mathrm{p} \approx 2$ (Bird et al., 1969; Takeuchi and Argon, 1976).

It is generally accepted that class M creep is controlled by dislocation climb (Bird et al., 1969; Takeuchi and Argon, 1976; Nix and Illschner, 1980). As first pointed out by Weertman (1975), theories for dislocation climb 
creep always predict a stress exponent of $\mathrm{n}=3$ if no special assumptions are made irrespective of the microstructural basis for the model. Therefore, it was hypothesized that there is a natural or universal creep law which predicts that $n=3$ for class $M$ creep. In reality, experimental observations consistently reveal that $n>3$ for most materials undergoing class $\mathrm{M}$ creep (Bird et al., 1969). In contrast, the exponential creep law is even more poorly understood in terms of dislocation processes operating in this regime. Some of the proposed mechanisms are discussed below.

\subsection{Assessment of Dislocation Core Diffusion-Controlled Creep Mechanisms}

If the activation energy is plotted as a function of temperature, it is often seen that $Q_{c} \approx Q_{1}$, where $Q_{1}$ is the activation energy for lattice diffusion, for $\mathrm{T}>0.7 \mathrm{~T}_{\mathrm{m}}$ and $\mathrm{Q}_{\mathrm{c}}<\mathrm{Q}_{1}$ for $\mathrm{T}<0.7 \mathrm{~T}_{\mathrm{m}}$ (Sherby and Burke, 1967; Lüthy et al., 1980). Interestingly, early research suggested that $Q_{c} \approx 0.5$ to $0.6 Q_{1}$ for some materials at intermediate temperatures (Fig. 3) (Barrett and Sherby, 1964; Robinson and Sherby, 1969; Lüthy et al., 1980). Sherby et al., (Barrett and Sherby, 1964; Robinson and Sherby, 1969; Lüthy et al., 1980) rationalized these observations on the basis that creep behavior at these temperatures is also controlled by dislocation climb, where vacancy diffusion now occurs along dislocation cores instead of through the lattice, so that $Q_{c} \approx Q_{p}$, where $Q_{p}$ is the activation energy for dislocation pipe or core diffusion. Thus, creep processes controlled by dislocation climb at intermediate and high temperatures were termed low temperature (L.T.) and high temperature (H.T.) climb, respectively (Frost and Ashby, 1975, 1977, 1982). Since dislocation core diffusion is dependent on the dislocation density (Hart, 1957; Shewmon, 1963), and $\rho$ is proportional to $\sigma^{2}$, the predicted stress exponent for L.T. climb is $(n+2)$ (Robinson and Sherby, 1969; Sherby and Young, 1975). Thus, the creep rate equation for L.T. climb can be specifically expressed as

$$
\dot{\varepsilon}_{\text {L.T. }}=A_{\text {L.T. }}\left(D_{0 p} G b / k T\right)(\sigma / G)^{\mathrm{n}+2} \exp \left(-Q_{p} / R T\right)
$$

and that for H.T. climb as

$$
\dot{\varepsilon}_{\text {H.T. }}=\mathrm{A}_{\text {H.T. }}\left(\mathrm{D}_{01} \mathrm{~Gb} / \mathrm{kT}\right)(\sigma / \mathrm{G})^{\mathrm{n}} \exp \left(-\mathrm{Q}_{1} / \mathrm{RT}\right)
$$

where $\mathrm{D}_{01}$ and $\mathrm{D}_{0 \mathrm{p}}$ are the frequency factors for lattice and pipe diffusion, respectively, and $\mathrm{A}_{\text {H.T. }}$ and $\mathrm{A}_{\mathrm{L} . \mathrm{T} \text {. }}$ are dimensionless constants.

Figure 4 schematically illustrates the regions of dominance of H.T. climb, L.T. climb and exponential creep on a plot of normalized creep rate, $\dot{k} \mathrm{kT} / \mathrm{D}_{\text {eff }} \mathrm{Gb}$, against $\sigma / \mathrm{G}$, where $\mathrm{D}_{\text {eff }}$ is an effective diffusion coefficient repre-

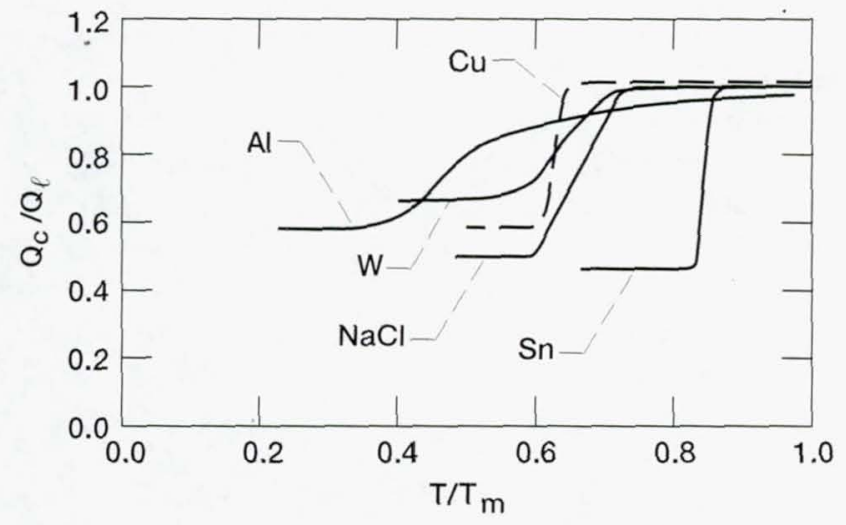

Figure 3.- Variation of the activation energy for creep, normalized by the activation energy for lattice selfdiffusion, with the homologous temperature for different materials showing the probable dominance of low temperature climb at low and intermediate temperatures (Liithy et al., 1980) (with kind permission from Elsevier Science Ltd.). 


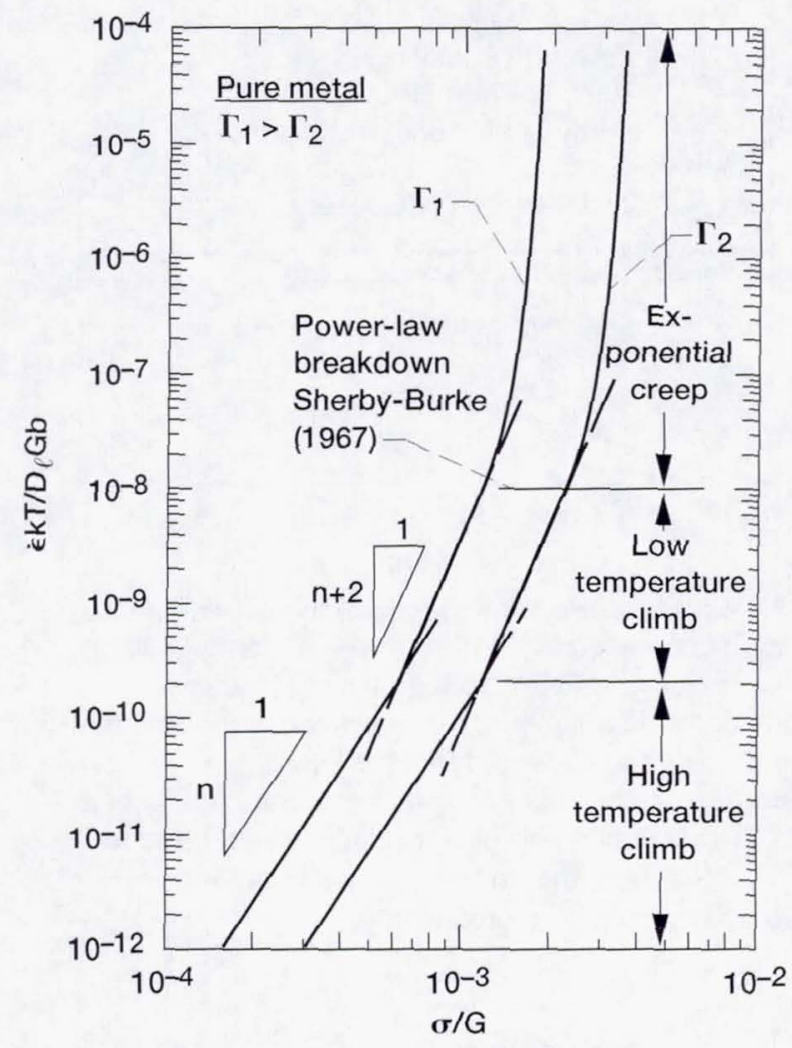

Figure 4.-Schematic illustration of normalized creep rate versus normalized stress for high $\left(\Gamma_{1}\right)$ and low $\left(\boldsymbol{\Gamma}_{2}\right)$ stacking fault energy pure metals showing the regions of class $\mathrm{M}$ creep (high temperature and low temperature climb) and exponential creep.

senting the combined effects due to lattice and core diffusion. The two sets of curves shown in Fig. 4 represent the creep behavior of high and low stacking fault energy materials. Sherby and Burke (SB) (1967) first suggested that the power-law breaks down at almost a constant value of $\dot{\varepsilon} / \mathrm{D}_{\text {eff }} \approx 10^{13} \mathrm{~m}^{2}$ (i.e., $\dot{\varepsilon} \mathrm{kT} / \mathrm{D}_{\mathrm{eff}} \mathrm{Gb} \approx 10^{-8}$ ) for many materials, and this criterion is indicated in Fig. 4.

Although the L.T. climb process predicts a stress exponent of $(n+2)$ and $Q_{c} \approx Q_{p}$, Spingarn et al., (1979) concluded from a reevaluation of the creep data for several materials that increases in the stress exponents did not correlate with $Q_{c} \approx Q_{p}$. Furthermore, the predicted creep rates based on theories for core diffusion were about 20 to 400 times slower than the experimental values for four metals: Al, Cu, Ni and W. Similarly, Kassner and Oldani (1988) and Kassner (1989) concluded that the $\mathrm{Q}_{c}$ versus T plot for $\mathrm{Ag}$ does not show any plateau corresponding to $\mathrm{Q}_{\mathrm{c}} \approx \mathrm{Q}_{\mathrm{p}}$. More recently, a detailed study on the creep of Cu also failed to reveal any evidence of L.T. climb-controlled creep in this material (Raj and Langdon, 1989, 1991(a),(b)). In this case, the activation energy was observed to decrease linearly with increasing values of $\sigma / G$, and it was demonstrated that this stress dependency of $Q_{c}$ could not be attributed to a transition from H.T. to L.T. climb with increasing normalized stress. Furthermore, it was demonstrated through a compilation of $\mathrm{Q}_{\mathrm{c}}$ values published in the literature that the activation energy data for $\mathrm{Cu}$ does not exhibit the simple step-function transition shown in Fig. 3 (Raj and Langdon, 1989).

Similarly, a plot of $\mathrm{Q}_{\mathrm{c}}$ against $\mathrm{T} / \mathrm{T} \mathrm{m}$ for $\mathrm{NaCl}$ polycrystals (Le Comte, 1965) and single crystals (Raj and Pharr, 1986(b)) shows no clear evidence of a plateau at intermediate temperatures (Fig. 5). Instead, $Q_{c}$ increases monotonically with increasing homologous temperature. The horizontal broken lines show the values for $\mathrm{Q}_{\mathrm{Cl}^{-}}$and $\mathrm{Q}_{\mathrm{Na}}{ }^{+}$, where $\mathrm{Q}_{\mathrm{Cl}}{ }^{-}$and $\mathrm{Q}_{\mathrm{Na}}{ }^{+}$are the average activation energies for the lattice diffusion of $\mathrm{Cl}^{-}$and $\mathrm{Na}^{+}$ions, respectively, in $\mathrm{NaCl}$ determined by replotting diffusion data obtained from several sources (Harrison et al., 1958; Barr et al., 1960; Barr et al., 1965; Beniere et al., 1970; Downing et al., 1970; Nelson and Friauf, 1970; Rothman et al., 1972; Mitchell and Lazarus, 1975; Beniere et al., 1977; Ho and Pratt, 1983). Figure 5 also shows the predicted 


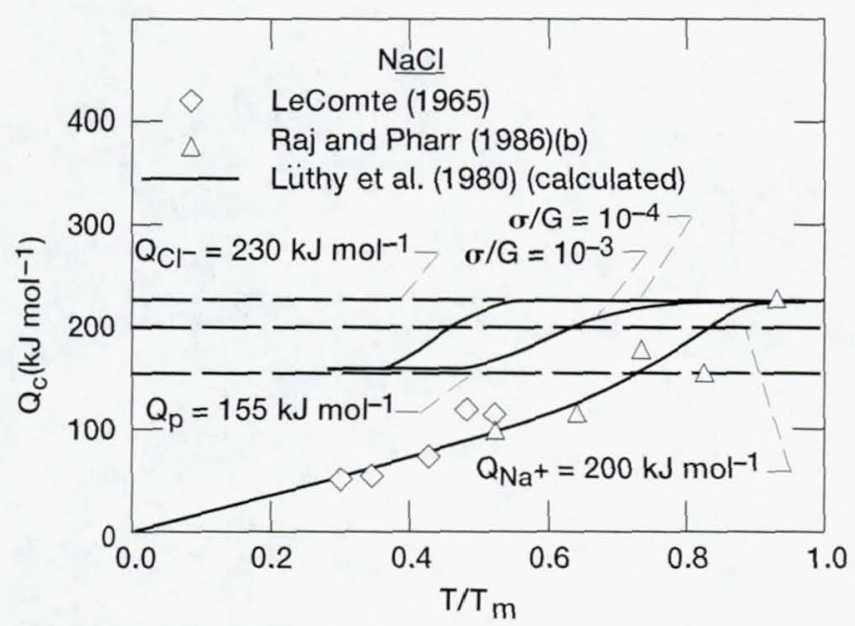

Figure 5.-Temperature dependence of the experimental activation energies for creep of poly- (Le-Comte, 1965) and single (Raj and Pharr, 1986 (b)) crystalline sodium chloride showing an absence of a plateau at intermediate temperatures. The expected trend from low to high temperature climb is also shown in the figure (Liithy et al, 1980). The activation energies for the lattice self-diffusion of $\mathrm{Cl}^{-}$and $\mathrm{Na}^{+}$, and that for pipe diffusion are represented by the horizontal broken lines.

variations in the temperature dependence of $\mathrm{Q}_{\mathrm{c}}$ assuming there is a transition from low to high temperature climb with increasing temperature for $\sigma / \mathrm{G}=10^{-4}$ and $10^{-3}$. The mean values of $\mathrm{Q}_{\mathrm{c}}$ are given by (Langdon and Mohamed, 1977)

$$
\mathrm{Q}_{\mathrm{c}}=\left(\mathrm{Q}_{1} \dot{\varepsilon}_{\text {H.T. }}+\mathrm{Q}_{\mathrm{p}} \dot{\varepsilon}_{\text {L.T. } .}\right) /\left(\dot{\varepsilon}_{\text {H.T. }}+\dot{\varepsilon}_{\text {L.T. }}\right)
$$

The calculated values of $Q_{c}$ were obtained using values of $Q_{p} \approx 155 \mathrm{~kJ} \mathrm{~mol}^{-1}$ (Ho and Pratt, 1983) and $Q_{1}=Q_{\mathrm{Cl}^{-}}$ $\approx 230 \mathrm{~kJ} \mathrm{~mol}^{-1}$ (Raj and Pharr, 1989).

Therefore, there is an increasing body of evidence which suggests that creep involving dislocation core diffusion is not a valid concept for many materials. It can then be inferred from Fig. 4 that in the absence of L.T. climb, there is a direct transition from class $\mathrm{M}$ to exponential creep. This implies that the mechanism(s) dominant in the exponential creep region is identical to those controlling creep at intermediate temperatures, where the transition from class $\mathrm{M}$ to the exponential creep involves a change over from a diffusional to a nondiffusional process(s). Therefore, the term "class M creep" as used in this chapter will specifically refer to H.T. climb dominated creep.

Alternatively, the low values of $\mathrm{Q}_{\mathrm{c}}<\mathrm{Q}_{1}$ observed at intermediate temperatures have been attributed to one or more non-diffusional processes (Dorn and Jaffe, 1961; Jaffe and Dorn, 1962; Poirier, 1976; Caillard and Martin, 1987; Raj and Langdon, 1991(b)). Most of these mechanisms predict a stress-dependent activation energy which leads to an exponential creep behavior similar to equation (4) or, if a power-law relation is used to analyze the data, to values of $n \gg 4$. Among the several mechanisms that have been proposed (Caillard and Martin, 1987; Raj and Langdon, 1991(b)), those involving the cross-slip of screw dislocations (Friedel, 1959, 1964, 1977; Dorn and Jaffe, 1961; Jaffe and Dorn, 1962; Poirier, 1976; Caillard and Martin, 1987; Carrard and Martin, 1987, 1988) and obstaclecontrolled glide (Nix and Ilschner, 1980; Raj and Langdon, 1991(b)) appear to be important at intermediate temperatures and high stresses.

\subsection{Nondiffusional Creep Mechanisms}

Several nondiffusional creep mechanisms have been proposed and these are reviewed elsewhere (Caillard and Martin, 1987; Raj and Langdon, 1991(b)). However, only two of these, namely cross-slip and obstacle- 
controlled glide mechanisms, appear to be relevant in most instances (Caillard and Martin, 1987; Nix and Illschner, 1980; Raj and Langdon, 1991(b)). These are discussed below.

\subsubsection{Cross-slip mechanisms}

The cross-slip of screw-oriented dislocations can lead to a decrease in the dislocation density through the annihilation of screw dislocations of opposite signs. Thus, it is possible for cross-slip to act as a recovery process in addition to its role as a multiplicative mechanism. The mechanism is complex and no good creep model exists to describe the process. As a result, there is some controversy as to whether this process is dominant at intermediate and high temperatures (Poirier, 1976, 1978, 1979; Sherby and Weertman, 1979). This controversy hinges on a fundamental question: Do edge and screw components of a dislocation loop move independently or sequentially during deformation? The conventional viewpoint, first advocated by Weertman (1955), assumes that the edge and screw components move sequentially with the rate of climb of edge dislocations being slower than the rate of cross-slip of screw dislocations. Therefore, the creep rate was controlled by dislocation climb in the Weertman model. The justification for this rationale lies in the fact that $Q_{c} \approx Q_{1}$ for experimental data reported for several materials in the class M creep regime (Sherby and Burke, 1967; Bird et al., 1969). In contrast, Poirier (1976, 1978) reanalyzed some of the published creep data for many metals and concluded that $Q_{c} \approx Q_{1}$ only in certain stress and temperature ranges and that $Q_{c}>Q_{1}$ at higher temperatures and lower stresses. He attributed these high values of $Q_{c}$ to creep controlled by cross-slip of screw dislocations, and proposed a creep model, where climb of the edge and cross-slip of screw components were assumed to occur as independent mechanisms (Poirier, 1976). The model also predicts that $\mathrm{Q}_{\mathrm{c}}<$ $\mathrm{Q}_{1}$ at very high stresses and low temperatures.

Theories involving cross-slip fall into two broad categories depending on whether the partial dislocations constrict along a length equal to that of a Frank-Read source (Schoeck and Seeger, 1955; Wolf, 1960; Püschl and Schoeck, 1993) or to a length less than the Frank-Read source (Friedel, 1959, 1964, 1977; Escaig, 1968(a) and (b); Bonneville and Escaig, 1979; Duesbery et al., 1992) prior to cross-slip onto the new slip plane. In each case, the unit dislocation dissociates into its partials in the cross-slip plane. The former process is energetically unfavorable by comparison with the latter so that it is generally not important. Two variations of the second process exist both of which are based on the Friedel model (Friedel, 1959, 1964, 1977). Jaffe and Dorn (1962) were the first to propose that the values of $Q_{c}<Q_{1}$ observed at intermediate temperatures (Fig. 3) could be rationalized using the Friedel cross-slip model. They assumed that the partial dislocations constrict over a critical length of about $4 \mathrm{~d}_{\mathrm{cs}}$, where $\mathrm{d}_{\mathrm{cs}}$ is the stress dependent stacking fault width. The activation energy for cross-slip, $Q_{c s}$, for this mechanism is (Jaffe and Dorn, 1962)

$$
\mathrm{Q}_{\mathrm{cs}}=\left(0.014 \beta^{\prime} \mathrm{Gb}^{3} / \mathrm{Np}\right)(\mathrm{G} / \sigma)^{0.5}\left[\ln \left\{\left(0.89 / \mathrm{N}_{\mathrm{P}}\right)(\mathrm{G} / \sigma)^{0.5}\right\}\right]^{0.5}
$$

where $\beta^{\prime}$ is a constant which is approximately 1.0 and 2.0 for low and high stacking fault energy materials, respectively, and $\mathrm{N}_{\mathrm{P}}$ is the number of dislocations in a piled-up array. Equation (8) predicts a nonlinear dependence of $\mathrm{Q}_{\mathrm{cs}}$ on stress.

In the second version, Escaig and others (Escaig, 1968(a), (b); Poirier, 1976; Bonneville and Escaig, 1979) assumed that the partial dislocations cross-slip at preexisting constrictions. However, as the constricted dislocation cross-slips and dissociates spontaneously into its partials in the cross-slip plane, two constricted nodes are formed at the intersection of the primary and cross-slip planes. The movement of these nodes away from each other as the partials glide on the new slip plane provides the driving force for cross-slip. An analysis of this process results in a complex expression for $\mathrm{Q}_{\mathrm{cS}}$ which depends on the magnitudes of the local stresses acting on the partials. A simplified relationship for this process leads to a linear dependence of $\mathrm{Q}_{\mathrm{cS}}$ on stress given by (Escaig, 1968(a) and (b), Poirier, 1976)

$$
\mathrm{Q}_{\mathrm{cs}}=\left(\mathrm{G}^{2} \mathrm{~b}^{4} / 1875 \Gamma\right)[\ln (\mathrm{Gb} / 14.5 \Gamma)]^{0.5}(1-3 \mathrm{~b} \sigma / 2 \Gamma)
$$

or

$$
\mathrm{Q}_{\mathrm{cS}}=\mathrm{Q}_{0}\left(1-\sigma \mathrm{V}^{*}\right)
$$

where $\mathrm{Q}_{0}$ is the maximum activation energy for cross-slip given by 


$$
\mathrm{Q}_{\mathrm{cS}}=5 \times 10^{-4}\left(\mathrm { Gb } ^ { 3 } ( \mathrm { Gb } / \Gamma ) \left(\ln (0.07 \mathrm{~Gb} / \Gamma)^{0.5}\right.\right.
$$

and the apparent activation volume, $\mathrm{V}^{*}$, by

$$
\mathrm{V}^{*}=3 \mathrm{bQ}_{0} / 2 \Gamma
$$

Note that $\mathrm{V}^{*}=\mathrm{A}^{*} \mathrm{~b}$, where $\mathrm{A}^{*}$ is the activation area for deformation.

Application of equations (8) and (9) to creep data obtained on polycrystalline Cu (Fig. 6) (Raj and Langdon, 1991(b)) showed no good agreement between theory and experiment. For $\mathrm{Cu}, \mathrm{Q}_{\mathrm{c}}$ decreases linearly with increasing values of $\sigma / \mathrm{G}$ and the nonlinear trend predicted by equation (8) is inconsistent with experimental observations (Fig. 6). Although equation (9) predicts this linear dependence of $Q_{c}$, it underestimates the experimental values by more than a factor of three in the case of $\mathrm{Cu}$ (Fig. 6) (Raj and Langdon, 1991(b)). In the case of NaCl, the Jaffe-Dorn cross-slip model given by equation (8) is reasonably close to the experimental data for $\mathrm{N}_{\mathrm{p}} \approx 5$ and $\beta^{\prime}=1$ (Fig. 7) (Raj and Pharr, 1986(b)). In contrast, the Escaig model underestimates $Q_{c}$ by a factor of four to twenty. Experimental observations of wavy slip morphology in $\mathrm{Al}$ (Carrard and Martin, 1987, 1988), Cu (Raj and Langdon, 1991(a)) and $\mathrm{NaCl}$ (Wawersik, 1984; Haasen et al., 1986; Skrotzki and Haasen, 1988) clearly suggest that cross-slip processes are important during creep. In the case of $\mathrm{Cu}$, it was observed that wavy slip occurs at high stresses and high temperatures close to the transition region between class $\mathrm{M}$ and exponential creep (Raj and Langdon, 1991(a)). In this case, since wavy slip was observed only after a finite amount of deformation, it appears that a critical combination of stress, temperature and strain conditions must be satisfied for these slip features to occur during creep. If wavy slip can be solely attributed to the cross-slip of screw dislocations, then the existence of these criteria can be qualitatively understood if it is assumed that the local stress required to constrict the partial dislocations to form a unit dislocation attains a critical value only at high temperatures and high strains. This criteria is more likely to be attained in high stacking fault energy materials, such as $\mathrm{Al}$, than in materials with lower values of $\Gamma$, such as $\mathrm{Cu}$.

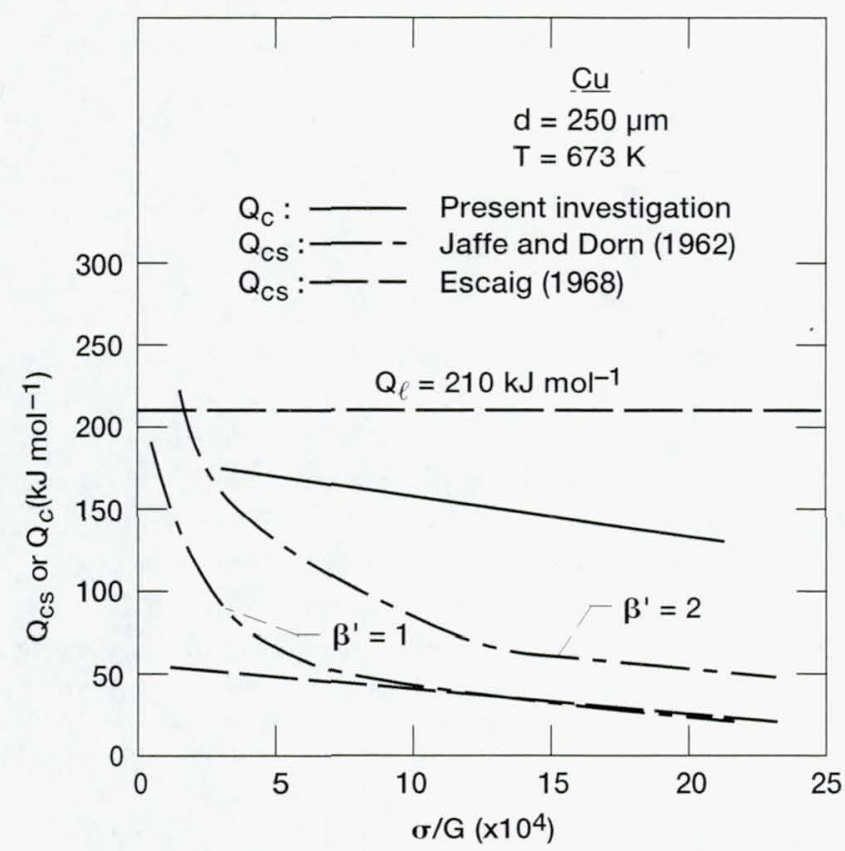

Figure 6.-Comparison of the experimental activation energies for copper with those predicted by the crossslip models. The values of $\beta^{\prime}=1$ and 2 are for low and high stacking fault energies, respectively (Raj and Langdon, 1991 (a)) (with kind permission from Elsevier Science Ltd.). 
In the absence of good theoretical models for cross-slip, empirical equations are often used to force a fit through the data, where it is necessary to make an a priori assumption that cross-slip is the dominant deformation mechanism in order to obtain the fitting constants. Nix et al., (1985(a) and (b)) expressed the activation free energy for cross-slip, $\Delta \mathrm{G}_{\mathrm{cs}}$, as

$$
\exp \left(-\Delta \mathrm{G}_{\mathrm{CS}} / \mathrm{kT}\right)=\exp \left(-\mathrm{Q}_{01} / \mathrm{kT}\right)\{\exp (\tau \mathrm{bA} * / \mathrm{kT})-1\}
$$

where $\mathrm{Q}_{01}$ is the activation energy for cross-slip at zero stress. The $\exp (\tau \mathrm{bA} * / \mathrm{kT})$ term in equation $(10)$ represents the activation energy for cross-slip events in the direction of the resolved shear stress while -1 represents the activation energy for dislocation motion in the opposite direction. Nix et al., (1985(a), (b)) assumed that $A^{*}=\alpha \cdot Q_{01} / G$, where $\alpha^{\prime}$ is a constant depending on the stacking fault energy of the material. Both $Q_{01}$ and $\alpha^{\prime}$ are adjustable . parameters which must be determined from experimental data assuming that cross-slip is the dominant mechanism. Referring to Fig. 7 and assuming typical values of $\mathrm{G}$ and that cross-slip is dominant in the linear region, $\mathrm{Q}_{01} \approx 185 \mathrm{~kJ} \mathrm{~mol}^{-1}$ for $\mathrm{NaCl}$ if the probability of backjumps is assumed to be zero (i.e. the '- 1 ' is deleted from equation (10)). Since $A^{*} \approx 300 b^{2}$ for cross-slip (Conrad, 1964; Evans and Rawlings, 1969; Bonneville and Escaig, 1979), the magnitude of $\alpha^{\prime} \approx 500$ to 950 between 300 and $973 \mathrm{~K}$.

\subsubsection{Obstacle-Controlled Glide of Dislocations}

Nix and Ilschner (1980) suggested that exponential creep is dominated by thermally activated obstaclelimited dislocation glide (Kocks et al., 1975; Frost and Ashby, 1982), where a gliding dislocation is obstructed by immobile or "forest" dislocations in single phase materials. Dislocation motion is limited by the cutting of these obstacles. The rate equation for this mechanism, assuming "rectangular" obstacles, is given by (Nix and Ilschner, 1980)

$$
\dot{\varepsilon}=\left(4 \times 10^{12} / \mathrm{M}^{3}\right)(\sigma / \mathrm{G})^{2} \exp \left[-\Delta \mathrm{F} / \mathrm{kT}\left\{1-\left(\sigma-\sigma_{\mathrm{b}}\right) / \sigma_{\mathrm{obs}}\right\}\right]
$$

where $\mathrm{M}$ is the Taylor factor, $\Delta \mathrm{F}$ is Helmholtz free energy required to move a dislocation past an obstacle without aid from an external stress, $\sigma_{\mathrm{b}}$ is the back stress on the dislocation and $\sigma_{\text {obs }}$ is the obstacle strength. The quantity $\sigma-\sigma_{b}$ represents an effective stress on the dislocation. The magnitude of $\Delta F$, which depends on the type of obstacle, is typically 0.2 to $1.0 \mathrm{~Gb}^{3}$ for medium strength obstacles such as forest dislocations (Frost and Ashby, 1982). Typical, values of $\Delta \mathrm{F}=0.4$ to $0.6 \mathrm{~Gb}^{3}$ appear to describe the experimental data on $\mathrm{Cu}$ (Fig. 8) (Raj and Langdon, 1991(b)) and $\mathrm{NaCl}$ (Fig. 7) (Raj and Pharr, 1986(b)) reasonably well. The magnitude of $\sigma_{\text {obs }}$ is given by (Taylor, 1934)

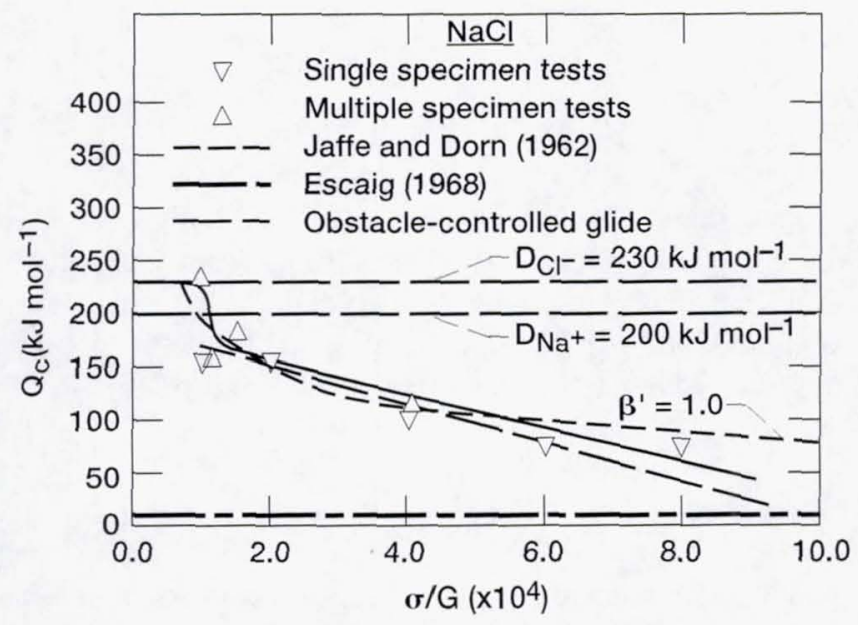

Figure 7.-Comparison of the stress dependence of the experimental activation energies for creep of $\mathrm{NaCl}$ single crystals with those predicted by the Escaig-Poirier crossslip creep model (Escaig, 1968 (b); Poirier, 1976) and the obstacle glide-controlled creep model (Nix and Ilschner, 1980). 


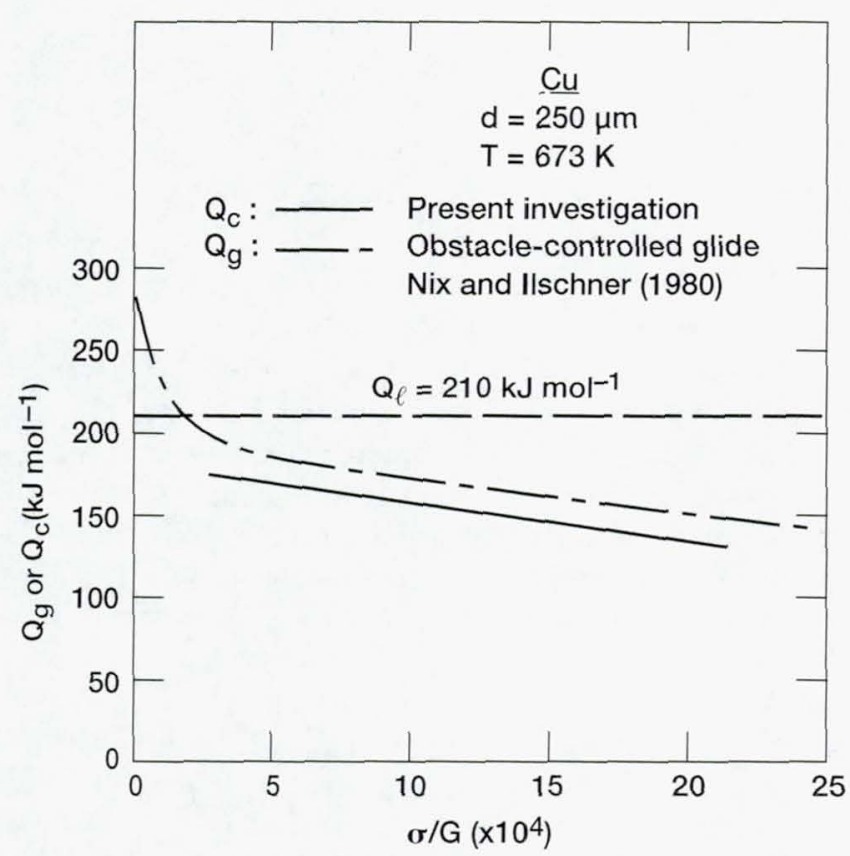

Figure 8.-Comparison of the experimental activation energies for copper with those predicted by the obstacle glide-controlled creep model (Raj and Langdon, 1991 (b)) (with kind permission from Elsevier Science Ltd.).

$$
\sigma_{\text {obs }}=\left(\alpha_{1} \mathrm{M}\right) \mathrm{Gb}(\rho)^{0.5}
$$

where $\alpha_{1}$ is a geometric constant equal to about 0.5 when $M=2$ (Bird et al., 1969; Mecking and Kocks, 1981). Alternatively, equation (11) can be formulated in terms of $\mathrm{V}^{*}$ with

$$
\mathrm{V}^{*}=\Delta \mathrm{F} / \sigma_{\mathrm{obs}}
$$

Equation (11) gives

$$
\mathrm{Q}_{\mathrm{c}}=\Delta \mathrm{F}\left\{1-\left(\sigma-\sigma_{\mathrm{b}}\right) / \sigma_{\mathrm{obs}}\right\}
$$

As shown in Fig. 7 (Raj and Pharr, 1986(b)) and Fig. 8 (Raj and Langdon, 1991(b)), the magnitudes of $Q_{c}$ determined from equation (14) are in reasonable agreement with the experimental data for $\mathrm{NaCl}$ and $\mathrm{Cu}$, respectively. These predicted curves were calculated assuming $\sigma_{\mathrm{b}} \approx 0.5 \sigma$ for $\mathrm{Cu}$ and $\mathrm{NaCl}$, respectively. Figures 7 and 8 suggest a direct transition from $Q_{c} \approx Q_{1}$ to $Q_{c}<Q_{1}$ with increasing $\sigma / G$ for both these materials which is consistent with the observed transition from class $M$ to exponential creep (Figs. 9 and 10).

\subsection{Effect of Stacking Fault Energy on Class M Creep}

The effect of stacking fault energy on the creep behavior of metals and alloys has been studied extensively under class M creep conditions (Barrett and Sherby, 1965; Davies et al., 1965; Shalayev et al., 1969; Singh Deo and Barrett, 1969; Vandervoort, 1969; Oikawa and Karashima, 1971; Johnson et al., 1972; Kozyrskiy et al., 1972(a),(b), Mohamed and Langdon, 1974; Okrainets and Pishchak, 1979; Yang et al., 1987). As a result, it is now wellestablished that the creep rate decreases by approximately a factor of 6000 for about an order magnitude decrease in 


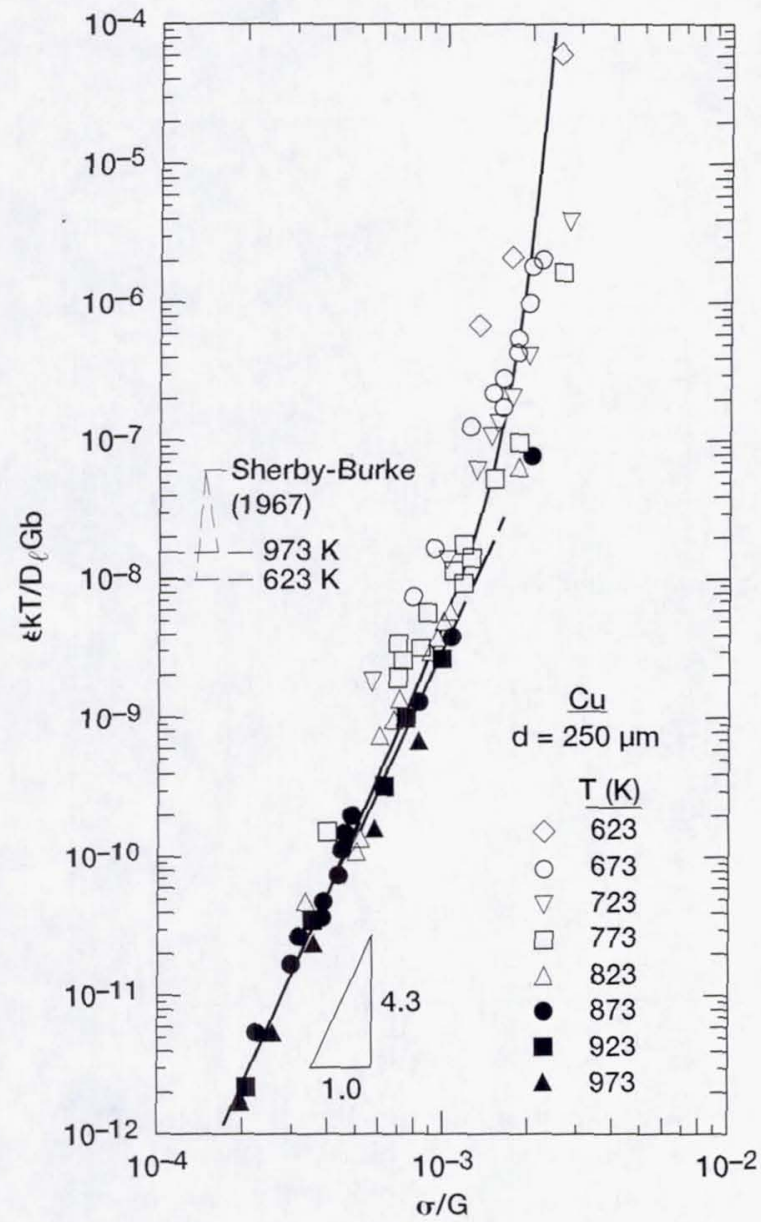

Figure 9.-Normalized creep rate versus normalized stress for copper showing the transition from class $M$ creep with $n \approx 4.3$ to exponential creep (Raj and Langdon, 1991 (b)) (with kind permission from Elsevier Science Ltd.).

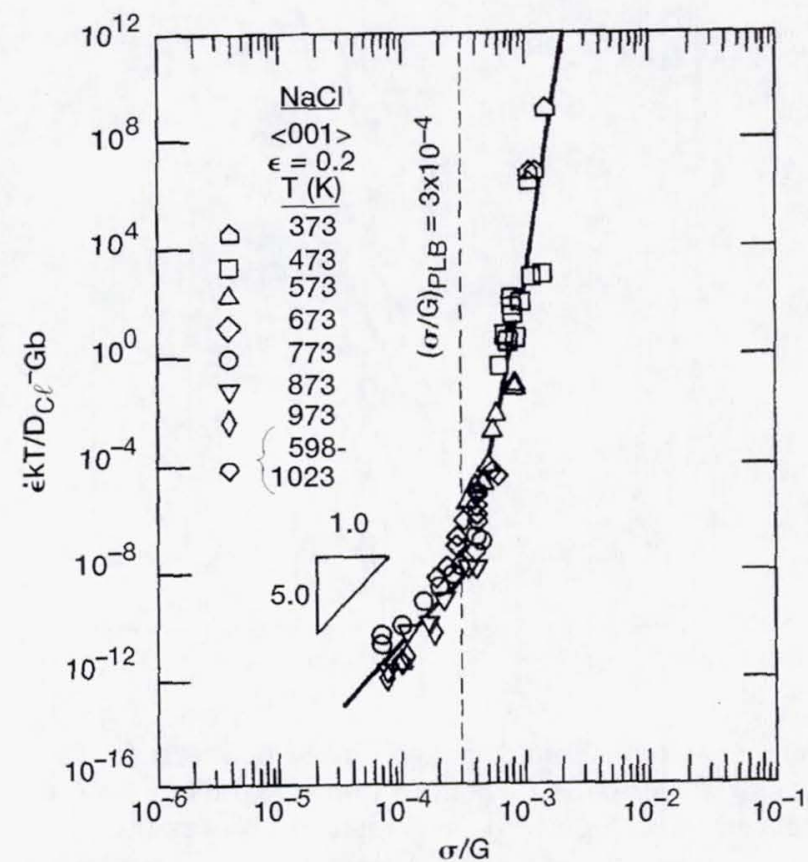

Figure 10.-Normalized creep rate versus normalized stress for sodium chloride single crystals showing the transition from class $M$ creep with $n \approx 5.0$ to exponential creep at $(\sigma / G)_{P L B}=3 \times 10^{-4}$ (Raj et al., 1989; Raj and Pharr, 1989) (with kind permission from Elsevier Science Ltd.).

$\Gamma$ (Barrett and Sherby, 1965). Stacking fault energy influences both dislocation climb (Weertman, 1965; Argon and Mofatt, 1981; Kong and Li, 1993) and cross-slip (see \$2.2.1) so that as $\Gamma / \mathrm{Gb}$ decreases, the rate of climb and crossslip decrease correspondingly.

The exact manner by which $\Gamma$ affects the parameters in the class $M$ creep equation (3) is poorly understood at present. However, most theoretical treatments of the problem assume that the extended dislocations form a constriction prior to cross-slip or climb so that the effect of $\Gamma$ enters the creep equation through its influence on the constriction energy. At present, both theory (Argon and Moffatt, 1981; Argon and Takeuchi, 1981; Kong and Li, 1993) and experiment (Barrett and Sherby, 1965; Mohamed and Langdon, 1974) suggest that the A parameter in equation (3) is a function of $(\Gamma / \mathrm{Gb})^{\mathrm{q}}$, where $\mathrm{q}$ is the stacking fault energy exponent.

Barrett and Sherby (1965) first proposed that $\dot{\varepsilon} \alpha \Gamma^{3.5}$ in the class M creep regime based on their experimental observations on pure metals. In a later extension of these results to other metals and alloys, Mohamed and Langdon (1974) observed that $\mathrm{q} \approx 3$. Several other values of $\mathrm{q}$ have also been reported in the literature varying between $\mathrm{q} \approx 1$ to 4 (e.g., Oikawa \& Karashima, 1971). In addition, alternative expressions to the power-law relation have been proposed. For example, Oikawa and Karashima (1971) observed $\dot{\varepsilon} \alpha \exp (\Gamma / \mathrm{Gb})$ for several copper alloys. However, the power-law relation with a value of $\mathrm{q} \approx 3$ is more commonly accepted in the literature. 


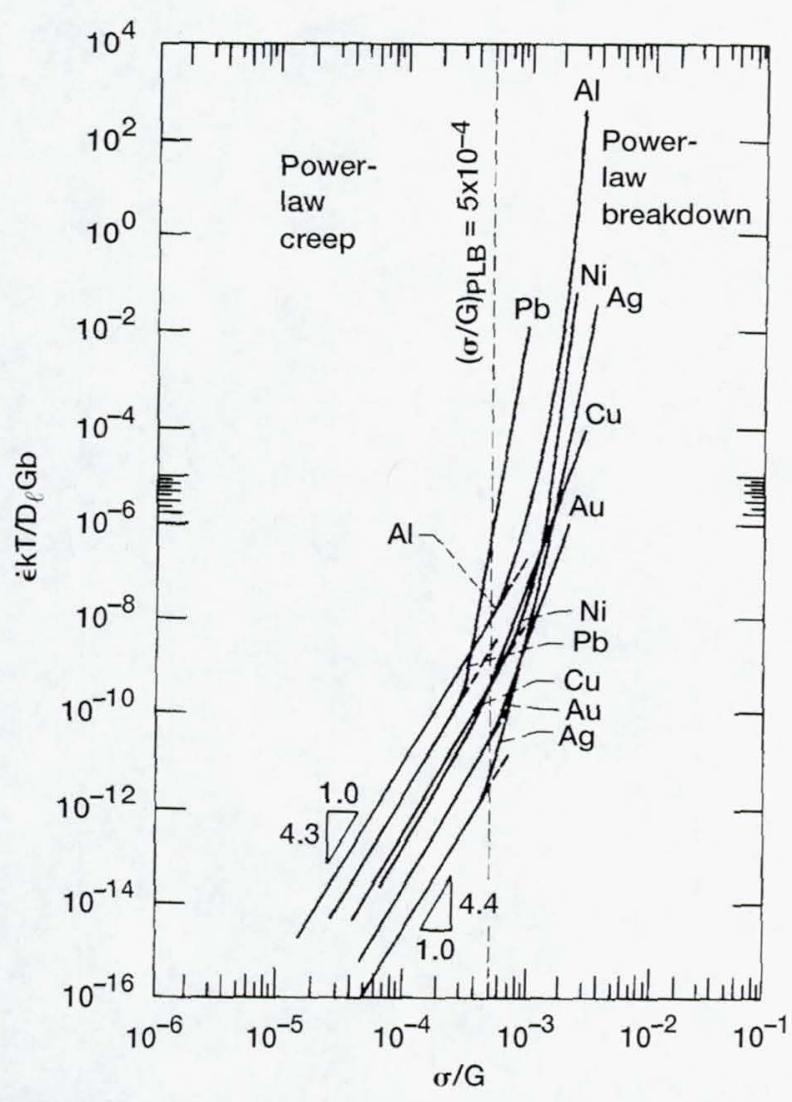

Figure 11.-Normalized creep rate versus normalized stress for several f.c.c. metals showing that the deviation from a class $M$ creep relation with a stress exponent of about 4.5 first occurs at $(\sigma / G))_{\mathrm{PLB}} \approx 5 \times 10^{-4}$ (Raj, 1986; Raj and Langdon, 1991 (b)) (with kind permission from Elsevier Science Ltd.).

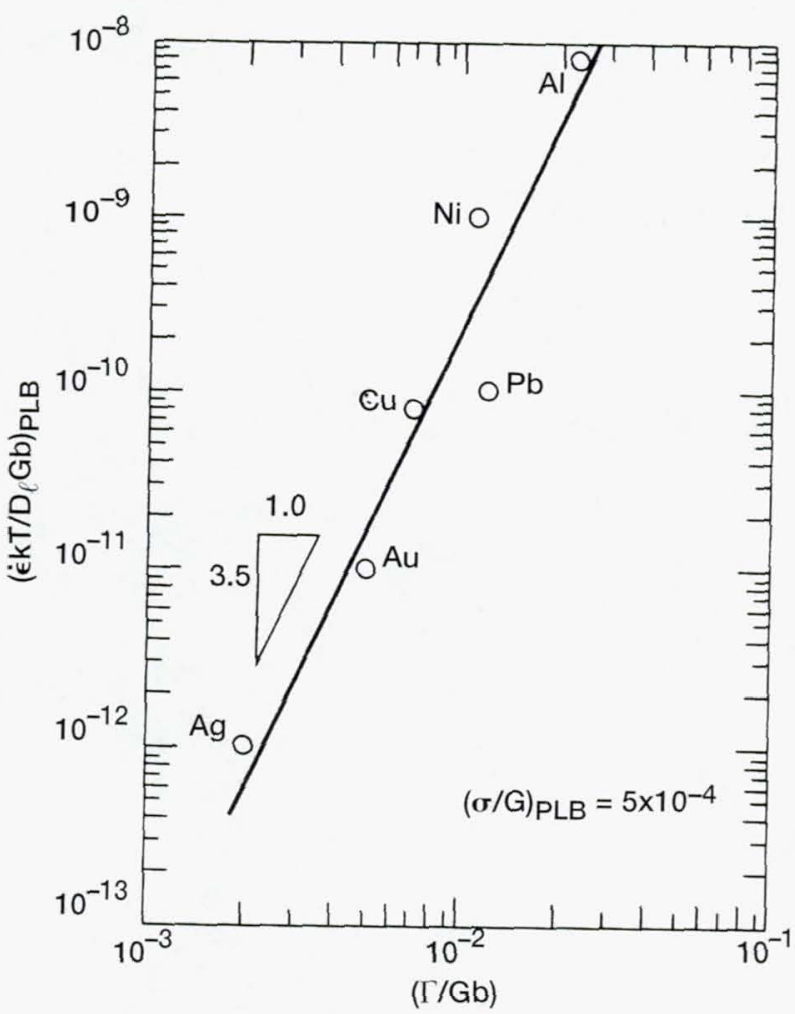

Figure 12.-Variation of the normalized creep rate at which the power-law breaks down for several f.c.c. metals (i.e. at $(\sigma / \mathrm{G})_{\mathrm{PLB}} \approx 5 \times 10^{-4}$ ) with the normalized stacking fault energy (Raj and Langdon, 1991 (b)) (with kind permission from Elsevier Science Ltd.).

\subsection{Power-Law Breakdown Criterion}

Since both the climb and cross-slip velocities are dependent on ( $/ \mathrm{Gb}$ ) (Poirier, 1976; Argon and Moffatt, 1981; Kong and Li, 1993), it is expected that recovery processes, and hence, the rate of evolution of the steady-state substructure should decrease with decreasing values of $\Gamma / \mathrm{Gb}$. Thus, nondiffusional creep mechanisms are likely to dominate at lower values of normalized creep rate with decreasing values of $\Gamma / \mathrm{Gb}$. As shown in Fig. 11, this is indeed the case when experimental creep data for a number of f.c.c. metals with $\Gamma / \mathrm{Gb}$ varying between $2.3 \times 10^{-2}$ for Al to $2 \times 10^{-3}$ for $\mathrm{Ag}$ are compared with each other on a normalized plot (Raj, 1986; Raj and Langdon, 1991(b)). These observations reveal that the normalized creep rates for $\mathrm{Al}$ are higher than those for $\mathrm{Ag}$ by as much as three to four orders of magnitude (Fig. 11). An important outcome of these results is that the power-law breaks down at about a constant value of $(\sigma / \mathrm{G})_{\mathrm{PLB}} \approx 5 \times 10^{-4}$ irrespective of the stacking fault energy. Similarly, $(\sigma / \mathrm{G})_{\mathrm{PLB}} \approx 3 \times 10^{-4}$ for $\mathrm{NaCl}$ single crystals (Fig. 10) (Raj and Pharr, 1989) and $5.5 \times 10^{-4}$ for an Al-3\% Mg alloy (Wang, at al. 1993). In contrast, the normalized creep rate, $\left(\dot{\varepsilon} \mathrm{kT} / \mathrm{D}_{1} \mathrm{~Gb}\right)_{\mathrm{PLB}}$, where $\mathrm{D}_{1}$ is the diffusion coefficient for lattice self diffusion, at which the power-law relation deviates from $n \approx 4.5$ for the pure metals decreases with decreasing $\Gamma / \mathrm{Gb}$ as ( $\dot{\varepsilon} \mathrm{kT} /$ $\left.\mathrm{D}_{1} \mathrm{~Gb}\right)_{\mathrm{PLB}} \alpha(\Gamma / \mathrm{Gb})^{3.5}$ (Fig. 12) (Raj and Langdon, 1991(b)). This exponent is similar to the values $\mathrm{q}=3.0$ to 3.5 observed in the class M creep regime (Barrett and Sherby, 1965; Mohamed and Langdon, 1974).

Three important points can be made regarding Figs. 11 and 12. First, the existence of an almost constant value of $(\sigma / \mathrm{G})_{\mathrm{PLB}}$ for these materials (Fig. 11) is significant since a number of substructural features depends on normalized stress (Bird et al., 1969; Takeuchi and Argon, 1976; Raj and Pharr, 1986(a)). Therefore, the results shown in Fig. 11 appear to imply that the power-law breaks down, at least, in part due to a change in one or more of 


TABLE II- - EFFECT OF STACKING FAULT
ENERGY ON THE TRANSITION TEMPERA-
TURE FOR SEVERAL MATERIALS.
(Raj and Langdon, 1991(b))
\begin{tabular}{|c|c|c|c|}
\hline Material & $\begin{array}{r}\Gamma, \\
\mathrm{mJ} \mathrm{m}{ }^{-2}\end{array}$ & $\Gamma / \mathrm{Gb}$ & $\mathrm{T}_{\mathrm{c}} / \mathrm{T}_{\mathrm{m}}$ \\
\hline $\mathrm{Al}$ & 200 & 23 & 0.55 \\
$\mathrm{Cu}$ & 55 & 7 & $>0.72$ \\
$\mathrm{Sn}$ & 3 & 0.6 & 0.85 \\
\hline
\end{tabular}

these micro-structural characteristics. Second, the decrease in $\left(\dot{\varepsilon} \mathrm{kT} / \mathrm{D}_{\mathrm{I}} \mathrm{Gb}\right)_{\mathrm{PLB}}$ with decreasing ( $\left.\Gamma / \mathrm{Gb}\right)$ (Fig. 12) can be attributed to the increasing dominance of nondiffusional creep mechanisms, such as obstacle-controlled glide, over H.T. climb at lower values of normalized creep rates in the low stacking fault energy metals. This is to be expected since it is well established that the extent of recovery decreases with decreasing stacking fault energy. The significant implication of this observation is that the transition temperature, $T_{c}$, above which $Q_{c} \approx Q_{1}$ in a plot of $Q_{c}$ against $\mathrm{T} / \mathrm{T}_{\mathrm{m}}$ is expected to increase as the stacking fault energy decreases. Indeed, as shown in Table II (Raj and Langdon, 1991(b)), this appears to be the case when the values of $\mathrm{T}_{\mathrm{c}}$ are compared with $\Gamma / \mathrm{Gb}$ for $\mathrm{Al}, \mathrm{Cu}$ and $\mathrm{Sn}$, but it is cautioned that more experimental data are required to confirm this trend. Third, the Sherby-Burke criterion, which predicts that the power-law breaks down at an almost constant value of $\dot{\varepsilon} / \mathrm{D}_{1} \approx 10^{13} \mathrm{~m}^{-2}$ (or equivalently, $\left(\dot{\varepsilon} \mathrm{kT} / \mathrm{D}_{1} \mathrm{~Gb}\right)_{\mathrm{PbB}} \approx 10^{-8}$ ) (Sherby and Burke, 1967), appears to be valid only for high stacking fault energy materials $\left(\Gamma>150 \mathrm{~J} \mathrm{~m}^{-2}\right.$ ) (Fig. 12) (Raj, 1986).

\subsection{SUBSTRUCTURE FORMATION IN NACL SINGLE CRYSTALS IN THE CLASS M AND EXPONENTIAL CREEP REGIMES}

Any attempt to develop realistic creep models must rest on a reasonable understanding of the processes leading to the formation and evolution of dislocation substructures during creep and the effect of stress, temperature and strain on the microstructure. These modeling efforts require detailed qualitative and quantitative characterization of the microstructure to provide a truer picture of creep. In particular, a microstructural characterization of both the class $\mathrm{M}$ and the exponential creep regions is essential in understanding why the power-law relation breaks down. For example, it is not clear at present whether the transition to an exponential stress dependence involves a sudden breakdown in the subgrain microstructure (Pharr, 1981) or whether it is due to a more gradual transformation in the substructure from a subgrain morphology present at high temperatures and low stresses to a microstructure consisting of dislocation tangles and cells characteristic of low temperature deformation (Michel et al., 1973; Challenger and Moteff, 1973; Kestenbach et al., 1976, 1978).

\subsection{Effect of Normalized Stress on Creep Substructure}

\subsubsection{Distinction Between Cells and Subgrains}

The terms "cells" (C) and "subgrains" (SG) have been traditionally used to describe the morphologies of certain low energy dislocation substructures formed as a result of the clustering of a uniform distribution of dislocations. The distinction between cells and subgrains is generally made with respect to differences in the appearance of the cell walls and the subgrain boundaries (Thompson, 1977). Cells consist of broad and diffused boundaries containing dislocation tangles. In contrast, subboundaries ( $\mathrm{Sb}$ ) are narrow and well defined, where the boundaries have a larger misorientation than the cell walls.

Figure 13(a) shows examples of these two substructural features in an etched $\mathrm{NaCl}$ single crystal after creep. The cell walls are resolvable into individual etch pits in contrast to the subboundaries. Each individual etch pit represents the point of termination of the dislocation line at the free surface. The cell boundary misorientation angle has been estimated to be about $0.1^{0}$ (Raj and Pharr, 1989). A close examination of Fig. 13 reveals that there are very few dislocations inside the cells. Two types of subboundaries, primary $(\mathrm{P})$ and secondary $(\mathrm{S})$, have been identified (Fig. 13(b)) (Raj and Pharr, 1989). In comparison to the primary subboundaries, where the etch pits along 

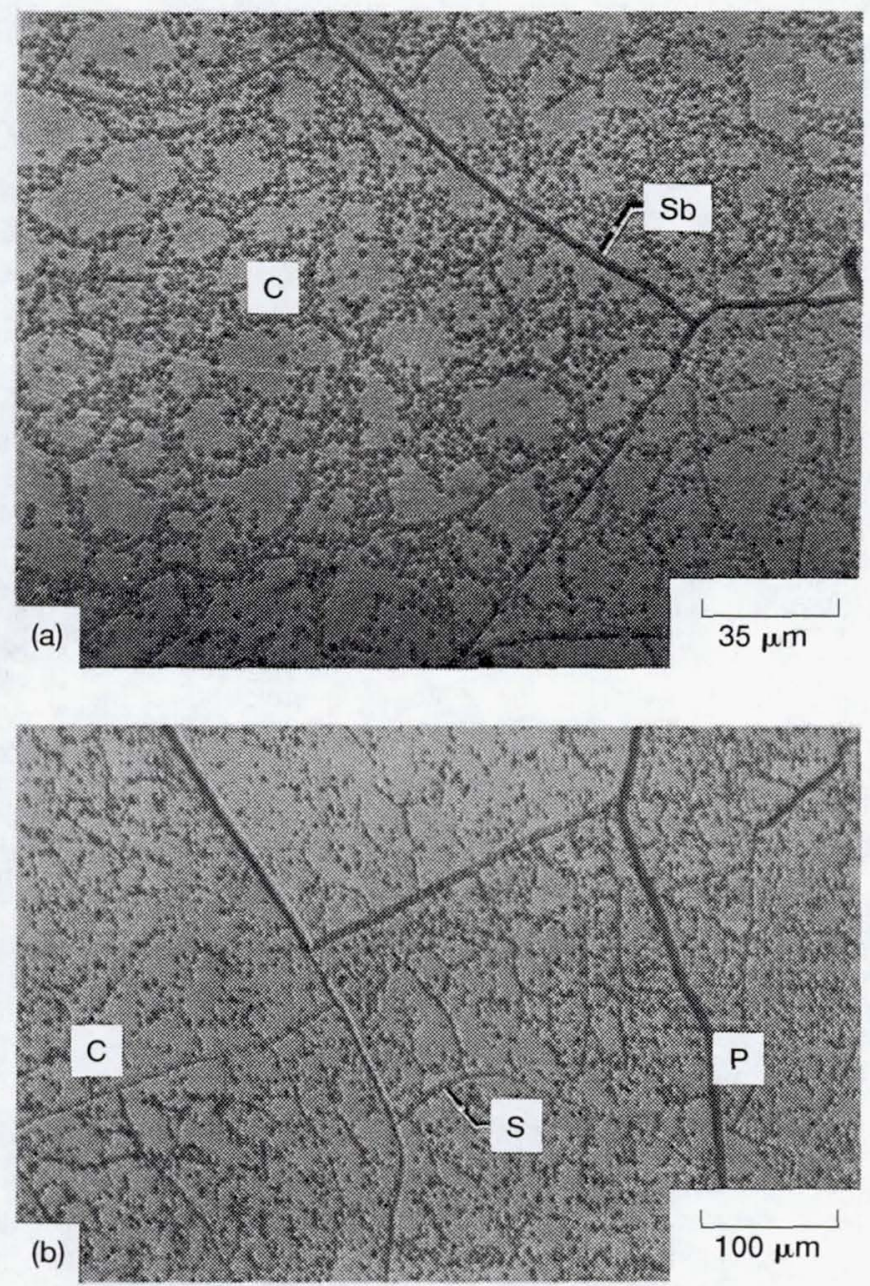

Figure 13.-Creep substructure in a $\mathrm{NaCl}$ single crystal specimen deformed to (a) a true strain of 0.9 at $873 \mathrm{~K}$ under a normalized stress of $2.2 \times 10^{-4}$ showing cells (C) and subboundaries (Sb); (b) a true strain of 0.37 at $973 \mathrm{~K}$ under a normalized stress of $7.5 \times 10^{-5}$ showing cells $(C)$, primary $(P)$, and secondary $(\mathrm{S})$ subboundaries (Raj and Pharr, 1989) (with kind permission from Elsevier Science Ltd.).

the boundary cannot not be distinguished individually at high magnification, the secondary subboundaries consist of individually distinct, but partially overlapping, etch pits. Thus, the primary subboundaries have a larger misorientation angle than the secondary subboundaries which in turn are more misoriented than the cell boundaries. Dislocation substructures may not always conform to these ideal definitions so that a precise identification of the microstructure may not always be possible in some instances. Additional difficulties may arise due to limitations of the observation technique used for studying the microstructures. For example, a cell boundary can be misidentified as a subboundary in transmission electron microscopy (TEM) due to the smaller field of view in the latter technique. For this reason, dislocation substructures are best studied using etch pit techniques (Takeuchi and Argon, 1976; Blum, 1993) although TEM has a decided advantage in the measurements of the boundary misorientation angles. As a result, there is some confusion in the use of these terms especially in the earlier literature, where the terms, "cells" and "subgrains", are used alternatively to describe the same substructural feature (e.g., Feltham and Sinclair, 1962, 1963). 


\subsubsection{Qualitative Descriptions of the Microstructures Formed in the Class $M$ and Exponential Creep Regions}

The fact that $(\sigma / \mathrm{G})_{\mathrm{PLB}}$ is similar for $\mathrm{NaCl}$ single crystals (Fig. 10) (Raj and Pharr, 1989) and for several f.c.c. metals (Fig. 11) (Raj, 1986; Raj and Langdon, 1991(b)) is interesting from the standpoint that many creep microstructural parameters, such as $d_{s}$ and $\rho$, are stress dependent. This observation suggests that the transition from class $\mathrm{M}$ to exponential creep with increasing values of normalized stress, $\sigma / \mathrm{G}$, and decreasing homologous temperatures, $T / T_{m}$, occurs when there is some critical transformation in the microstructure. Although there is now a wide body of compiled information available on creep substructure (Sherby and Burke, 1967; Bird et al., 1969; Takeuchi and Argon, 1976; Nix and Ilschner, 1980), most of these observations were conducted in a limited range of stresses and temperatures primarily corresponding to the class $\mathrm{M}$ creep regime. As a result, these early studies do not generally provide much information regarding the exponential creep region although they form the basis of our current understanding of class $\mathrm{M}$ creep.

Limited microstructural studies have been conducted on AISI 316 stainless steels in the class M and exponential creep regions (Michel et al., 1973; Challenger and Moteff, 1973; Kestenbach et al., 1976, 1978). These observations suggest that the equiaxed subgrain microstructure, formed at low stresses and high temperatures corresponding to the class $\mathrm{M}$ creep region, is replaced by a uniform distribution of dislocations at high stresses and low temperatures well within the exponential creep regime (Fig. 14) (Kestenbach et al., 1976, 1978). This transformation from a subgrain microstructure occurs gradually passing through several intermediate stages involving the formation of other substructural features, such as elongated subgrains and cells (Michel et al. 1973; Kestenbach et al., 1978),

Similar observations were also reported for $\mathrm{NaCl}$ single crystals deformed in the [001] direction under a constant compressive stress (Raj et al., 1989; Raj and Pharr, 1989). Large equiaxed primary subgrains were observed at $973 \mathrm{~K}$ and at a value of $\sigma / \mathrm{G}=7.5 \times 10^{-5}$ (Fig. 13 (b)) corresponding to the class $\mathrm{M}$ creep region in Fig. 10 Two other microstructural features are also visible within the primary subgrains: secondary subboundaries, which divide the primary subgrains by forming random interconnecting networks, and equiaxed cells, which are present within the secondary subgrains. These microstructures were generally clean and well defined in comparison to those produced at lower temperatures and higher stresses. True steady-state creep behavior was observed under these

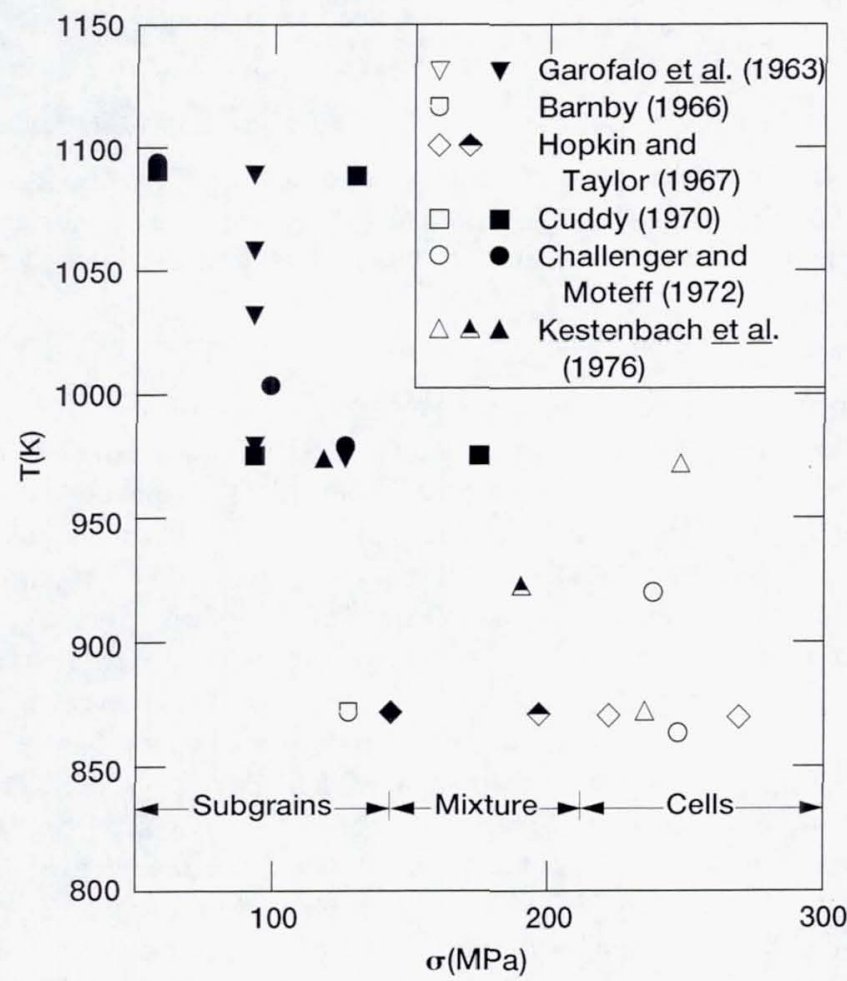

Figure 14.-Types of creep substructures formed in 316 stainless steels at different stresses and temperatures (Kestenbach et al., 1976) (courtesy Metallurgical and Materials Transactions). 

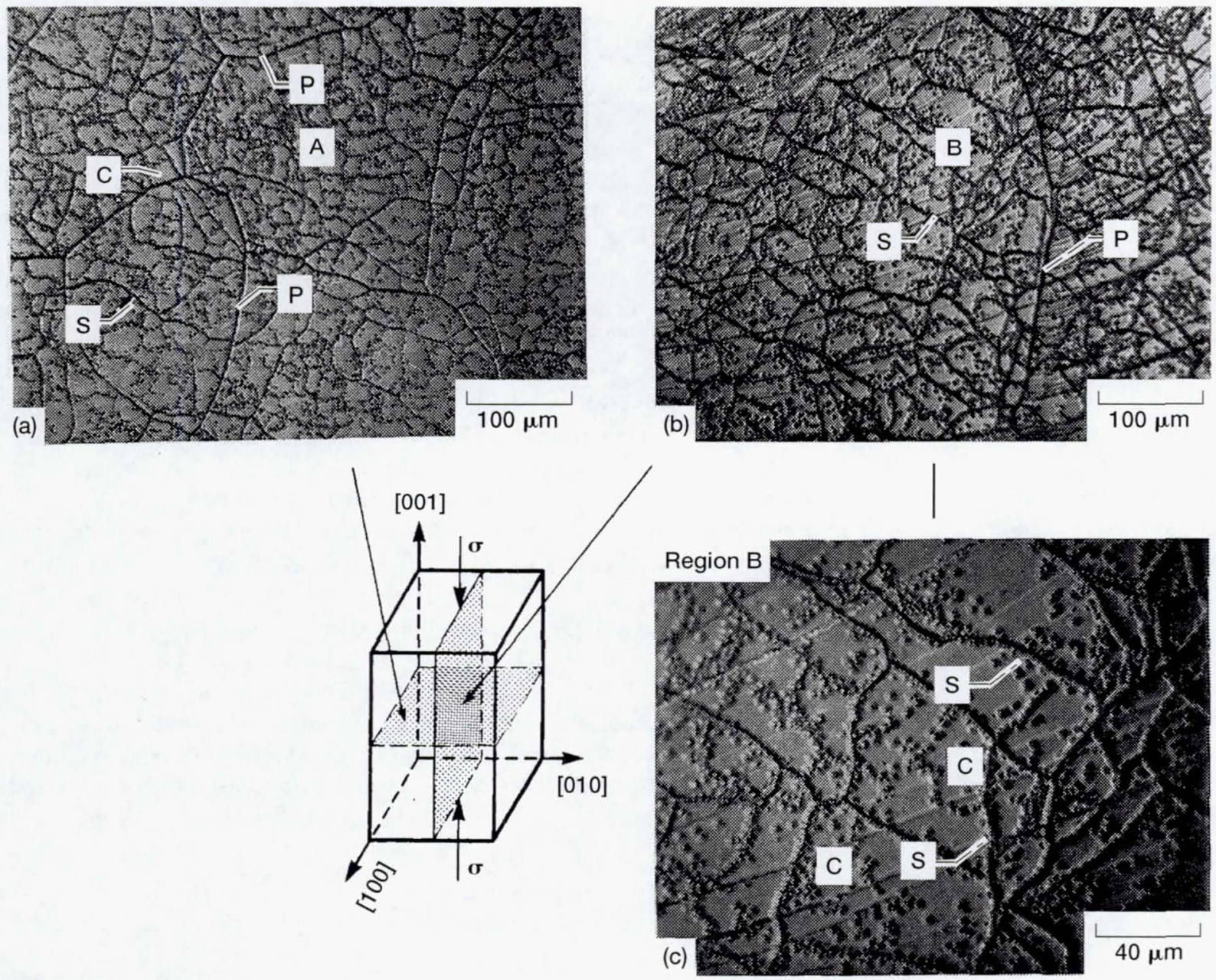

Figure 15.-Microstructure of a specimen deformed to a true strain of 0.35 at $973 \mathrm{~K}$ under a normalized stress of about $10^{-4}$ showing sections (a) transverse, and (b), (c) parallel to the stress axis; (c) is a high magnification view of region B in (b). An increase in the cell width (e.g. at A) is evident in (a) (Raj and Pharr, 1989) (with kind permission from Elsevier Science Ltd.).

conditions so that these microstructures represent steady-state creep substructures (Raj et al., 1989; Raj and Pharr, 1989). These secondary boundaries and cellular features are also evident in the published micrographs of creepdeformed $\mathrm{LiF}$ (Streb and Reppich, 1973) and $\mathrm{NaCl}$ (Eggeler and Blum, 1981) single crystals.

The primary and secondary boundaries also form at a higher value of $\sigma / \mathrm{G}=10^{-4}$ as observed in the sections transverse (Fig. 15 (a)) and parallel (Figs. 15(b) and (c)) to the stress axis. The longitudinal section was cleaved from the crystal and the cleavage facets are visible in Figs. 15(b) and (c). Equiaxed primary subgrains, subdivided by secondary subboundaries and cells, are visible in Fig. 15(a). Similarly, a single primary subboundary and several intercon-necting secondary subboundaries can be seen in Fig. 15(b). A high magnification view of region B in Fig. 15(b) shows that the cell boundaries consist of a loose configuration of etch pits whereas those in the secondary sub-boundaries are present as distinguishable but nonresolvable etch pits (Fig. 15 (c)). The similar microstructures observed in the transverse (Fig. 15(a)) and longitudinal (Figs. 15(b) and (c)) sections clearly demonstrate that these substructural features are representative of the bulk and that they are not due to any polishing artifact as suggested by Vogler et al., (1991). More importantly, cells have been reported to form in a large number of materials both in compression and tension creep thereby suggesting that they are not specific to $\mathrm{NaCl}$, testing conditions or metallographic preparation techniques (Gupta and Strutt, 1967; Hasegawa et al., 1970, 1971; Feltham and Sinclair, 1972; Streb and Reppich, 1973; Goel et al., 1983; Soliman et al., 1983; Ginter et al., 1984; Lee and Nam, 1988).

A comparison of Figs. 13(b) and 15(a) reveals that there is a significant increase in the width of a few cell boundaries within some of the primary subgrains with an increase in $\sigma / \mathrm{G}$. (e.g,. A in Fig. 15(a)). This widening of 
the cell boundaries becomes increasingly predominant with increasing normalized stresses. The increase in the width of the cell boundaries appears to be correlated with a corresponding decrease in the number of secondary subboundaries and a tendency towards nonsteady state creep behavior (Raj et al., 1989; Raj and Pharr, 1989). An example of this microstructural variation is shown in Fig. 16(a) for a specimen deformed at a value of $\sigma / \mathrm{G}=$ $2.2 \times 10^{-4}$, where it is seen that, while the primary subgrains are still equiaxed, the cell boundaries within many of them are extremely coarse (e.g., A in Fig. 16(a)) in contrast to with those shown in Fig. 13(b). In this case, secondary subboundaries were rarely observed. However, narrow cell boundaries and secondary subboundaries are evident within some of the primary subgrains in other regions of the specimen (e.g., B in Fig. 16(a)). It is clear from a comparison of Figs. 13(b) and 16(a) that the steady-state microstructure involves a refinement of the cell boundaries and the formation of secondary subboundaries. It is important to note that these microstructures are formed under class $\mathrm{M}$ creep conditions when $\mathrm{n} \approx 5$ (Fig. 10), although no real steady-state was attained when $\sigma / \mathrm{G}>10^{-4}$. These observations contradict the commonly-held opinion that subgrain formation is a sufficient condition for the occurrence of steady-state creep (Volger et al., 1991; Blum, 1993). Similarly, Mo single crystals did not exhibit steady-state creep behavior even after 42 percent strain despite the fact that a subgrain microstructure had developed during deformation (Clauer et al., 1970).

The first evidence of a change in the appearance of the primary subgrains occurs at a value of $\sigma / \mathrm{G}=$ $3.3 \times 10^{-4}$ which is close to the point of transition from class $\mathrm{M}$ to exponential creep (Fig. 10). In this case, some of the subgrains are more elongated (e.g. A in Fig. 16(b)) compared to others (e.g. B in Fig. 16(b)), and this increase is more pronounced at $\sigma / \mathrm{G}=4.0 \times 10^{-4}$ as is evident through a comparison of subgrains A and B in Fig. 16(c). These elongated subgrains exhibit a ladder-like morphology with their long boundaries oriented along the $\langle 110\rangle$ primary slip direction. Similar microstructures of elongated and equiaxed subgrains, often distributed in alternate bands of narrow and wide subgrains, have been observed in many materials (Gupta and Strutt, 1967, Clauer et al., 1970; Hasegawa et al., 1970, 1971; Feltham and Sinclair, 1972; Poirier, 1972; Orlová et al, 1972(a); Hüther and Reppich, 1973; Takeuchi and Argon, 1976; Kestenbach et al. 1978). Significantly, there is also a variation in the internal microstructures within these two types of subgrains. The elongated subgrains (e.g., A in Fig. 16(c)) have a higher dislocation density, a larger number of cells with coarser boundaries and fewer secondary subboundaries than their equiaxed neighbors (e.g., B in Fig. 16(c)). These observations suggest that there is an apparent correlation between the aspect ratio of a subgrain and its internal microstructure. Hasegawa et al. $(1970,1971)$ have reported similar observations on $\mathrm{Cu}$ single crystals, where the elongated subgrains appeared to creep at slower rates than the equiaxed ones. These results suggest that the elongated subgrains are harder than their equiaxed neighbors due to differences in their internal dislocation microstructure. Earlier observations have shown that the elongated subgrains tend to become equiaxed by subboundary migration (Hasegawa et al., 1970, 1971; Clauer et al., 1970).

At a value of $\sigma / \mathrm{G}=7.5 \times 10^{-4}$ and $473 \mathrm{~K}$, corresponding to well within the exponential creep region (Fig. 10), no cells and subgrains are observed below $\varepsilon \leq 0.2$ (Fig. 16(d)). Instead, the microstructure consists of an uniform distribution of dislocations interspersed with light patches of lower etch pit density and long subboundaries oriented approximately along the $\langle 100\rangle$ direction. These light patches of low dislocation density are probably the first indications of cell formation through dislocation annihilation since they were not observed at lower temperatures and higher stresses. However, well developed subgrains were observed at $\varepsilon \approx 1.0$ although their formation did not result in steady-state creep. At very high values of normalized stress corresponding to $\sigma / \mathrm{G}=1.4 \times 10^{-3}$, the microstructure consists primarily of a uniform distribution of dislocations and little or no subboundaries (Fig. 16(e)). The substructure in the exponential creep regime is strongly dependent on strain and temperature, where an increase in both these parameters tends to promote subgrain formation (Raj and Pharr, 1989).

The general picture that emerges from these results is that the transition from class $\mathrm{M}$ to exponential creep is associated with gradual variations in the microstructure which involve a complex and subtle interaction between different elements of the substructure. These substructural changes occur both at the coarser level of the primary subgrains as well as at the finer levels of the cells, secondary subboundaries and dislocations. Steady-state creep occurs when there is a dynamic equilibrium in the evolution of all these microstructural features. Figure 17 schematically summarizes the microstructural observations reported on $\mathrm{NaCl}$ single crystals (Raj et al., 1991). The lower portion of the figure illustrates the idealized microstructures formed at different values of normalized stress above and below $(\sigma / \mathrm{G})_{\mathrm{PLB}}$ while the upper portion correlates the major substructural processes occurring at each microstructure with a corresponding simplified geometrical representation. Figure 17 also lists the increasing probability of the recovery mechanisms which are likely to dominate at different values of normalized stress. Typical values of $\dot{\varepsilon} \mathrm{kT} / \mathrm{D}_{1} \mathrm{~Gb}$ are also included in Fig. 17. The effect of strain or temperature would be essentially to increase the probability of attaining this equilibrium substructure (i.e., from right to left in Fig. 17). 

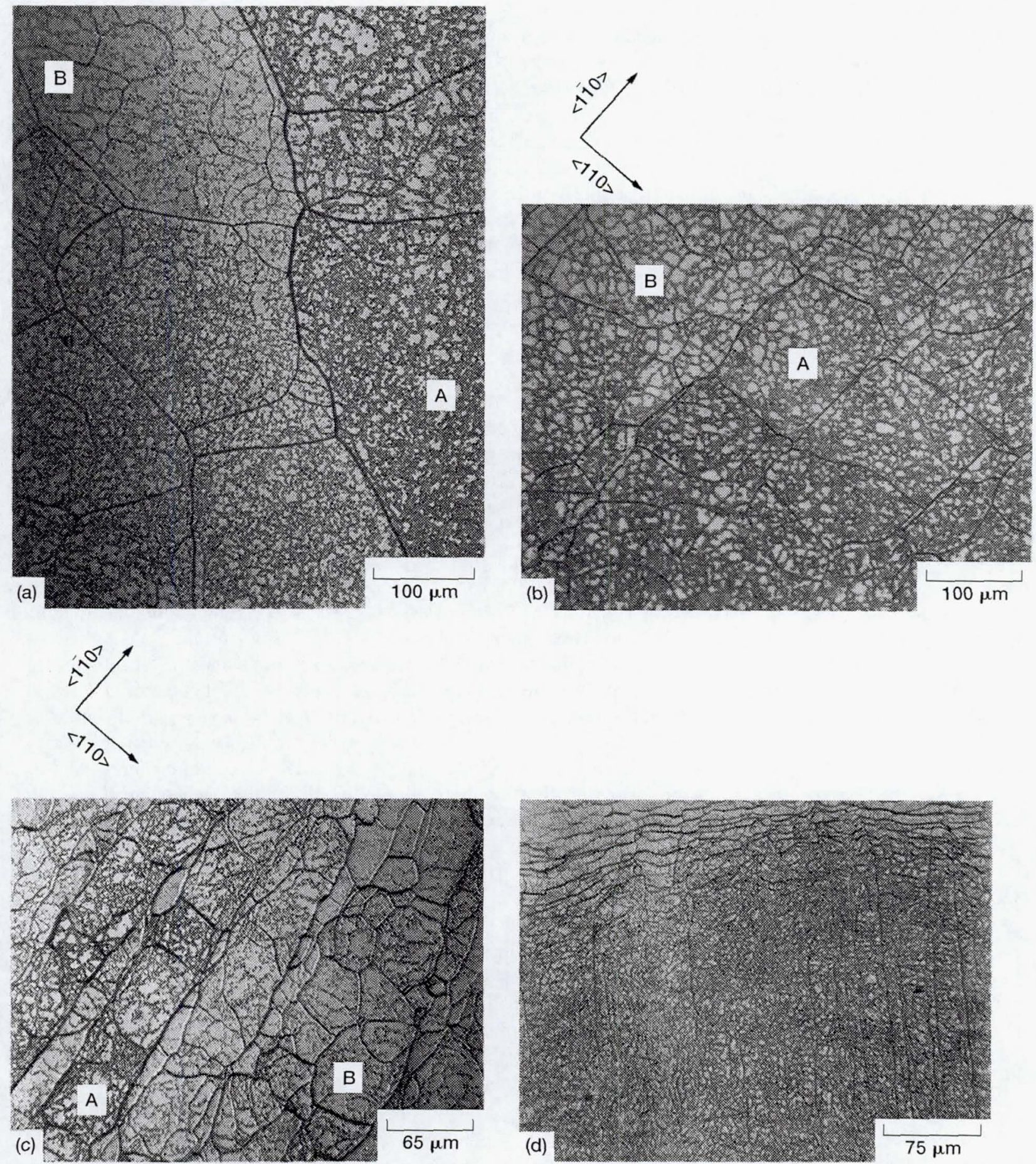

Figure 16.-Variation in the creep substructure in $\mathrm{NaCl}$ single crystals deformed at different values of normalized stress. (a) $\sigma / G \approx 2.2 \times 10^{-4}$; substructure in the class $M$ creep region showing that the cell boundaries are wider in subgrain $A$ than in subgrain $B$ although both primary subgrains are equiaxed. $(b) \sigma / G \approx 3.3 \times 10^{-4}$; elongated (A) and equiaxed $(B)$ subgrains containing coarser and narrower cell boundaries, respectively, are visible close to $(\sigma / G)_{P L B}$. (c) $\sigma / G \approx 4.0 \times 10^{-4}$; elongated $(A)$ and equiaxed $(B)$ primary subgrains showing different internal cellular and dislocation microstructures within them. The elongated subgrain boundaries are oriented along the $<110>$ crystallographic direction and these subgrains exhibit a "ladder-like" appearance. (d) $\sigma / G \approx 7.5 \times 10^{-4}$; microstructure of the exponential creep region showing long subboundaries and light patches in an otherwise uniform distribution of dislocations. 


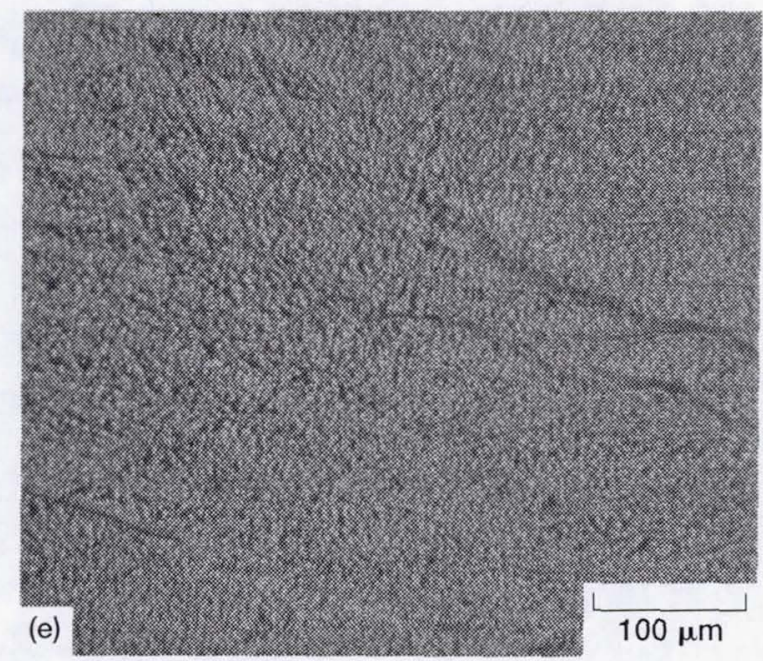

Figure 16.-(e) $\sigma / G \approx 1.4 \times 10^{-3}$; uniform distribution of dislocations (Raj et al., 1989; Raj and Pharr, 1989) (with kind permission from Elsevier Science Ltd.).

At high stresses and low temperatures corresponding to the exponential creep regime, the substructure consists predominantly of forest dislocations and a few subboundaries lying along secondary slip planes at low values of strain, and mainly of subgrains when $\varepsilon \geq 1$ (Fig. 17(a)). Glide polygonization can lead to the formation of the light patches with lower dislocation density. As mentioned earlier, these light patches appear to be the first evidence of cell formation. Other recovery processes, such as cross-slip and dynamic recrystallization, can be important depending on strain, temperature and stacking fault energy. At lower stresses and higher temperatures close to the powerlaw breakdown criterion, but still within the exponential creep regime, the substructure consists of a mixture of elongated ('hard') and equiaxed ('soft') subgrains (Fig. 17(b)). The elongated subgrains contain a high density of dislocations and coarse-walled cells. In contrast, the equiaxed subgrains have an internal microstructure of thinwalled cells, secondary subboundaries and fewer "free" dislocations. This difference in the internal microstructures of these two types of subgrains is expected to provide the driving force for subboundary migration which ultimately leads to the formation of equiaxed primary subgrains (Fig. 17(c)). There is now considerable evidence which suggests that subboundary migration can contribute typically between 3 to 25 percent to the total creep strain although contributions as much as 100 percent have also been reported (Exell and Warrington, 1972; Vollertsen et al., 1984; Caillard and Martin, 1987; Biberger and Blum, 1988, 1992(a and b)). Careful experiments suggest that subboundary migration occurs after large stress reductions which allows the microstructure to attain the equilibrium subgrain size characteristic of the reduced stress (Ferreira and Stang, 1979, 1983; Eggeler and Blum, 1981; Goel et al., 1983; Soliman et al., 1983; Mohamed et al., 1985).

Other recovery mechanisms, such as dislocation climb, cross-slip and subgrain rotation, are also expected to play an important role. The shape of the primary subgrains is essentially equiaxed and stable in the class $\mathrm{M}$ creep region but there are differences in their internal microstructures from one subgrain to another. Some contain a large number of thick-walled cells while others contain mainly thin-walled cells. Cell boundary refinement is dominant in this region leading to a narrowing of the cell walls. There is also a corresponding decrease in the dislocation density within the cells and an increase in the density of secondary subboundaries (Fig. 17(c)). Steady-state creep does not occur until cell boundary refinement is completed and a dynamic equilibrium exists between different elements of the microstructure (Fig. 17(d)). Under these conditions, the microstructure consists of equiaxed primary subgrains containing cells and a network of secondary subboundaries. The cell boundaries appear to be the major sources of dislocations since they are likely to breakup more easily than a subboundary to accommodate strain inhomogeneity in the material (Fig. 18) (Raj and Langdon, 1991(b)). Evidence that subboundaries are mechanically stable in contrast to cell boundaries at high stresses is presented in $\S 4$. Similar interpretations were also advanced by Gupta and Strutt (1967), who also suggested that cell boundary disintegration during creep provided mobile dislocations for deformation. 


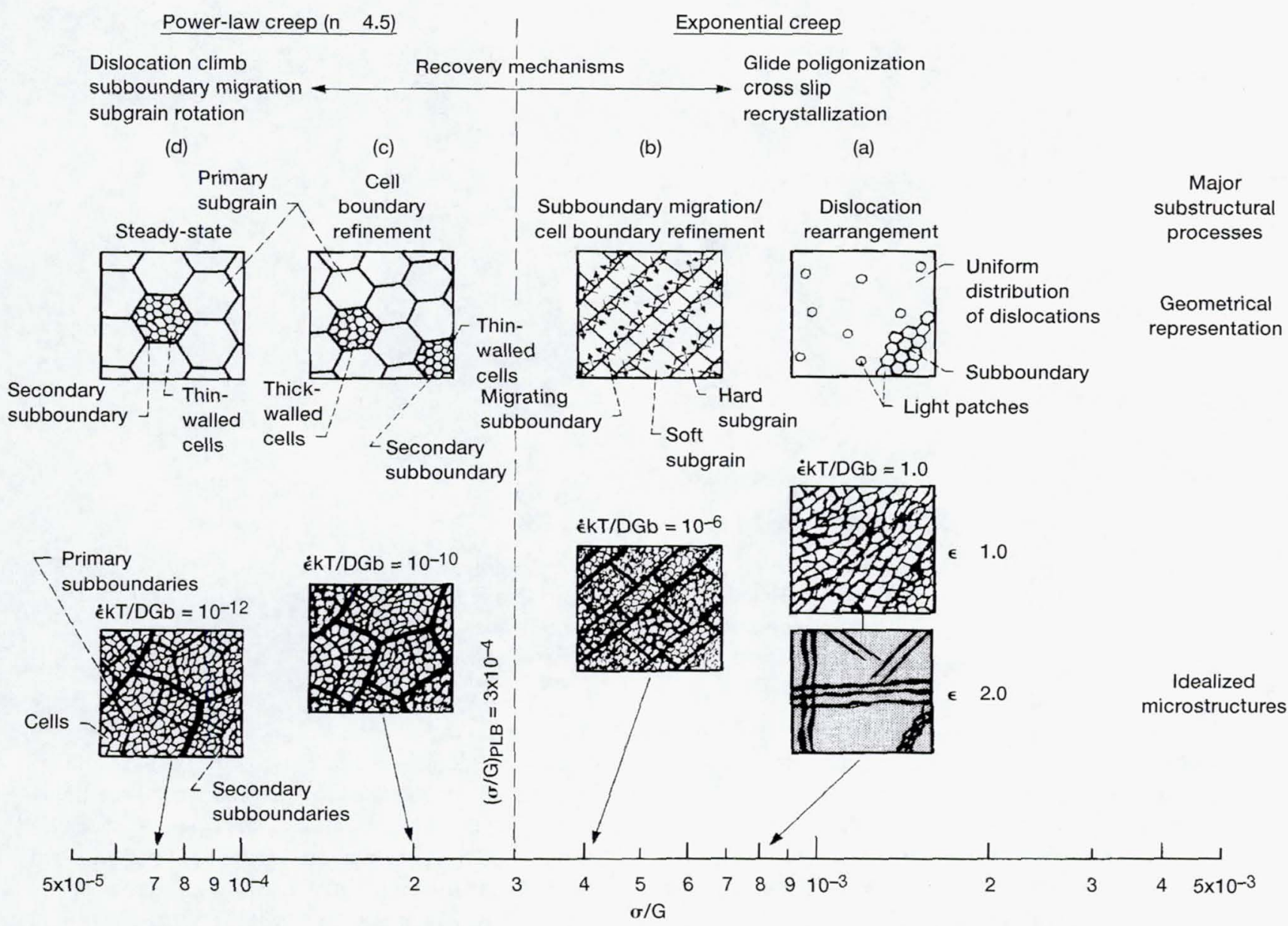

Figure 17.-Schematic idealized creep substructures, and their simplified geometric representation, formed in sodium chloride single crystals in the exponential and class $\mathrm{M}$ creep regions. The different recovery mechanisms which are likely to dominate under various creep conditions are also indicated. (a) A high density of dislocations interspersed with light patches and a few subboundaries form when $\epsilon<0.2$ but are transformed to well-formed subgrains when $\epsilon>1.0$ if recovery mechanisms are sufficiently rapid. Some materials may recrystallize if cross-slip and glide polygonization do not occur rapidly enough to bring about significant recovery. (b) Elongated and equiaxed subgrains with different internal cellular and dislocation microstructures result in subboundary migration. Cell boundary refinement probably occurs by cross-slip and dislocation climb. (c) Equiaxed subgrains, cell boundary refinement and secondary subboundary formation occur in the class $\mathrm{M}$ region. Steady-state creep is not observed. (d) An equilibrium substructure of equiaxed primary subgrains, secondary subboundaries and narrow-walled cells is observed under conditions where steady-state creep is observed.

\subsubsection{Quantitative Descriptions of the Stress Dependence of the Dislocation Substructure}

Figure 19 shows the variation of $d_{c} / b$ and $d_{s} / b$ with $\sigma / G$ for $\mathrm{NaCl}$ single crystals, where the data have been obtained at different temperatures and various values of strain (Raj et al., 1989; Raj and Pharr, 1989). The magnitude of $(\sigma / \mathrm{G})_{\mathrm{PLB}}$ is also indicated on the figure. Three points may be noted from Fig. 19. First, cells and subgrains, once formed, attain an equilibrium size inversely proportional and primarily determined by the normalized stress. The cell and subgrain sizes do not vary significantly with strain and temperature, which is consistent with previous observations (Bird et al., 1969). Second, subgrains and cells are stable even when $\sigma / G>(\sigma / G)_{P L B}$, thereby suggesting that the class $\mathrm{M}$ to exponential creep transition does not involve any catastrophic break-up of cells and subgrains as postulated by Pharr (1981). Third, the two plots appear to converge at a value of $\sigma / \mathrm{G}>2 \times 10^{-3}$. This convergence in the two plots is expected to occur when both $\mathrm{d}_{\mathrm{c}}$ and $\mathrm{d}_{\mathrm{s}}$ approach the average spacing between the "forest" dislocations i.e., $d_{c} \approx d_{s} \approx(\rho)^{-0.5}$.

It is evident from Fig. 19 that both $\mathrm{d}_{\mathrm{c}}$ and $\mathrm{d}_{\mathrm{s}}$ decrease with increasing stress in accordance with the experimentally-determined equations 


$$
\begin{aligned}
& \mathrm{d}_{\mathrm{c}} / \mathrm{b}=930(\mathrm{G} / \sigma)^{0.45} \\
& \mathrm{~d}_{\mathrm{s}} / \mathrm{b}=15(\mathrm{G} / \sigma)^{1.1}
\end{aligned}
$$

Equations (15a) and (b) suggest that the cell size exhibits a weaker dependence on the normalized stress than the subgrain size. Although cells have been observed in several materials (Gupta and Strutt, 1967; Hasegawa et al., 1970, 1971; Feltham and Sinclair, 1972; Streb and Reppich, 1973; Goel et al., 1983; Mohamed et al., 1985; Lee and Nam, 1988), quantitative information on the stress dependence of $d_{c}$ was not always reported. Previous measurements reported by Goel et al., (1983) on an Al-Zn alloy also suggest that $d_{c}$ is weakly dependent on the applied stress.

The stress dependence of $d_{s}\left(\right.$ or $\left.d_{c}\right)$ can be expressed in a general form as :

$$
d_{s} / b=K(G / \sigma)^{m}
$$
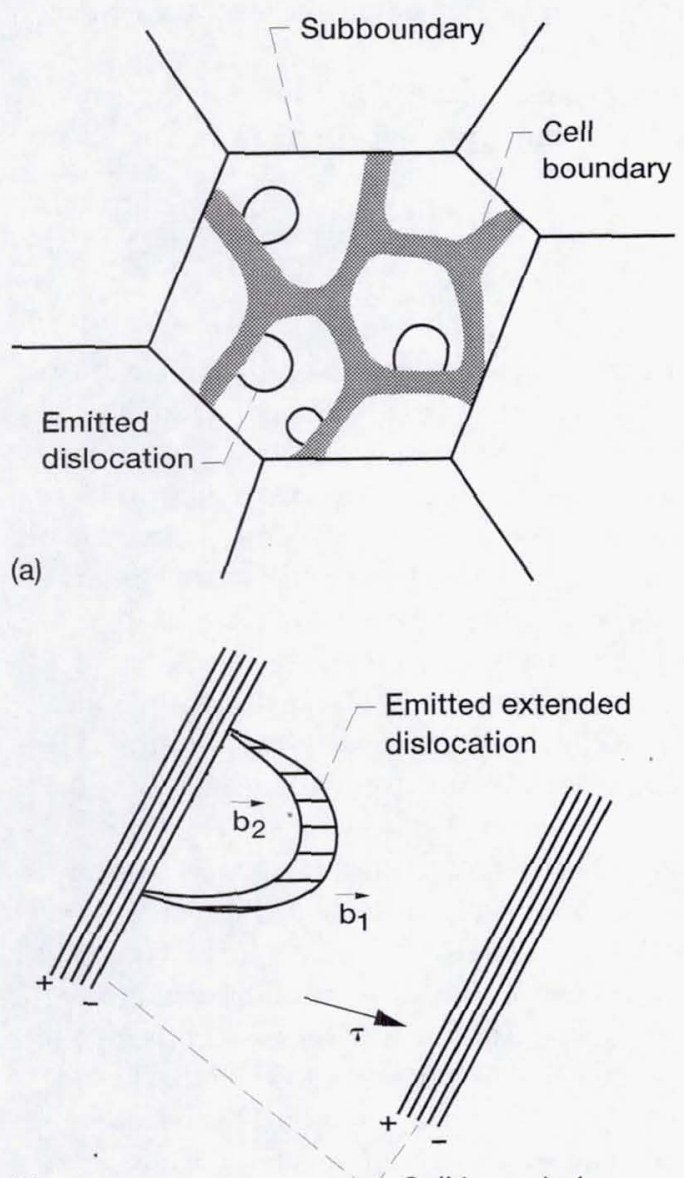

(b)

$\angle$ Cell boundaries

Figure 18.--Schematic showing (a) cells and dislocations within a primary subgrain and (b) emission of extended dislocations from a cell wall and its glide toward the opposite cell wall; $\overrightarrow{b_{1}}$ and $\vec{b}_{2}$ are the Burgers vectors of the partial dislocations (Raj and Langdon, 1991 (b)) (with kind permission from Elsevier Science Ltd.).

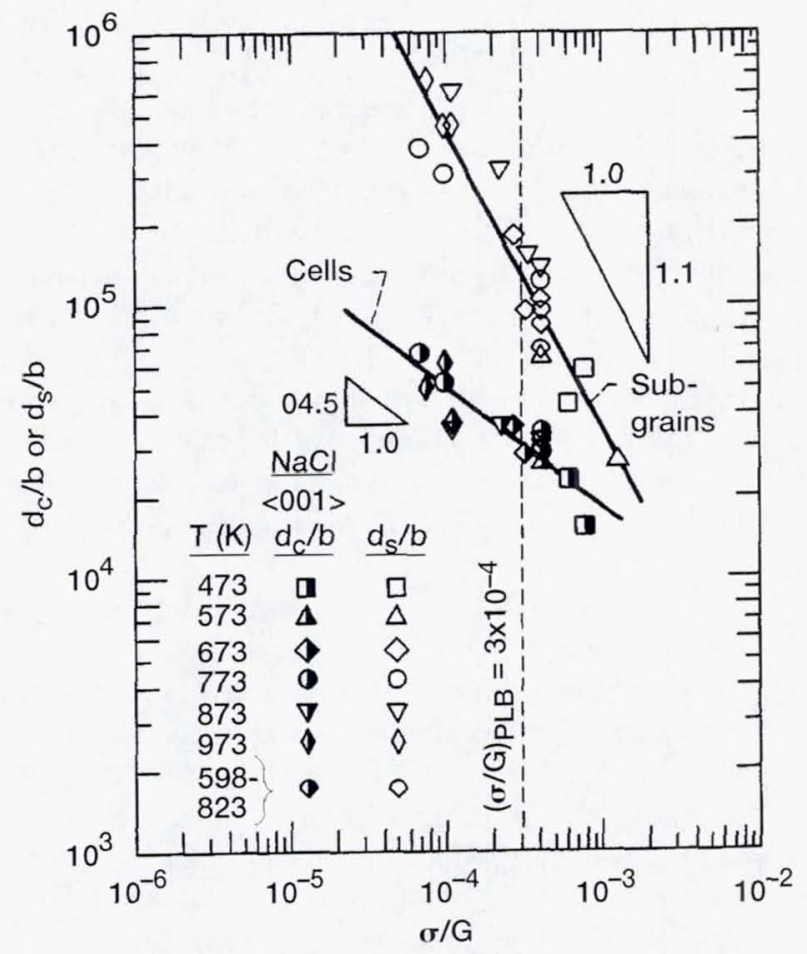

Figure 19.-Variations in the normalized cell and subgrain sizes with normalized stress for sodium chloride single crystals (Raj and Pharr, 1989) (with kind permission from Elsevier Science Ltd.). 
where $\mathrm{m}$ and $\mathrm{K}$ are constants. A comparison of equations (15b) and (16) suggests that $\mathrm{K}=15$ and $\mathrm{m}=1.1$. The magnitudes of these constants fall within the range of values reported for other ionic crystals (Raj and Pharr, 1986(a)). A compilation of the experimental values of $\mathrm{m}$ and $\mathrm{K}$ for several materials suggests that $0<\mathrm{m}<2$ and $0.01<\mathrm{K}<10^{5}$ (Raj and Pharr, 1986(a)). Although several values of $\mathrm{m}$ and $\mathrm{K}$ have been reported in the literature, it was demonstrated in an earlier study that these variations in the magnitudes of $\mathrm{m}$ and $\mathrm{K}$ are due to random error in the experimental measurements of subgrain size for most materials (Raj and Pharr, 1986(a)). As a result of this statistical scatter, the experimental values of $\mathrm{K}$ were shown to be dependent on $\mathrm{m}$ through

$$
\log K=-2.8 m+4.2
$$

so that $\mathrm{K} \approx 23$ when $\mathrm{m}=1$. These values were shown to fit most experimental data reasonably well so that equation (16) can be expressed as the universal relation

$$
\mathrm{d}_{\mathrm{s}} / \mathrm{b}=23(\mathrm{G} / \sigma)
$$

Owing to the unique relation between $\mathrm{m}$ and $\mathrm{K}$ expressed by equation (17), several other combinations of these constants exist which would predict almost identical values of $d_{s} / b$ for a constant magnitude of $\sigma / G$. Nevertheless, a value of $\mathrm{m}=1$ appears to be justified since experimental values of $\mathrm{m}$ approach unity whenever the number of meas-urements of the subgrain size exceeds 15 (Raj and Pharr, 1986(a)).

There is insufficient data relating $\mathrm{d}_{c} / \mathrm{b}$ with $\sigma / \mathrm{G}$ for high temperature creep in comparison to the vast amount of similar data on $d_{s}$. Thus, a universal relation, such as equation (18a), is unavailable. However, equation (15a) can be reformulated assuming $\mathrm{m}=0.5$ to give

$$
\mathrm{d}_{\mathrm{c}} / \mathrm{b} \approx 600(\mathrm{G} / \sigma)^{0.5}
$$

One reason for this apparent lack of data may be due to a misidentification of cells as subgrains in the literature. In addition, the terms "cells" and "subgrains" have been used interchangeably in the older literature (e.g. Feltham and Sinclair, 1962 to 1963) which results in confusion in the proper identification of the microstructure. The other possibility is that in some materials the random dislocations within the primary subgrains may prefer to cluster themselves into three-dimensional dislocation networks rather than cells. Thus, the clustering of random dislocations may be envisioned as shown schematically in Fig. 20. In this case, the formation of primary subgrains is always favored but additional dislocation rearrangement may sequentially favor the formation of either cells and secondary subboundaries or three-dimensional dislocation networks as the steady-state microstructure. Other microstructural features, such as microbands and recrystallized grains (Hansen and Jensen, 1991), can also form under certain deformation conditions so that the microstructural picture can be more complicated than that illustrated by Fig. 20. Ultimately, the type of microstructure formed will be dictated by the need to lower the free energy of the deforming solid.

As discussed in $\$ 2.4$, the magnitude of $(\sigma / \mathrm{G})_{\mathrm{PLB}}$ is approximately constant for many materials (Figs. 9 to 11) thereby suggesting that the transition from class $M$ to exponential creep is due to some definite microstructural changes occurring in the material. In an earlier study, Pharr (1981) proposed that this transition involves a catastrophic breakdown in the subgrain microstructure so that $(\sigma / \mathrm{G})_{\mathrm{PLB}}$ then represents a measure of the average strength of subboundaries in the material. However, the description of the microstructural changes occurring in the class $\mathrm{M}$ and exponential creep regions in $\$ 3.1 .2$ clearly suggest that there is no sudden breakdown in the subgrain microstructure, which in fact can extend to values of $\sigma / \mathrm{G}>(\sigma / \mathrm{G})_{\mathrm{PLB}}$. Instead, these observations suggest a gradual increase in the width of cell boundaries which appear to result in an elongated subgrain microstructure at the point of transition between class $M$ and exponential creep. Measurements of the average dimensions of the cell walls, $L$, and the cell interiors, $\mathrm{L}^{\mathrm{s}}$, obtained over a wide range of strain, stress and temperature reveal a remarkable correlation between the ratio, $\mathrm{L}^{\mathrm{h}} \mathrm{L}^{\mathrm{s}}$ and $(\sigma / \mathrm{G})_{\mathrm{PLB}}$ despite a large amount of scatter (Fig. 21) (Raj and Freed, 1992). This scatter is largely influenced by the effect of strain and temperature, both of which affect the degree of refinement of the cell boundaries (Raj and Pharr, 1986(b), 1992; Raj and Freed, 1992). Therefore, an increased tendency towards a steady-state microstructure appears to result as $\mathrm{L} / \mathrm{L} s$ tends towards a constant value of 0.2 assuming other elements of the substructure also attain dynamic equilibrium. Noting that the volume fraction of the cell boundaries, $\mathrm{f}^{\mathrm{cb}}$, is related to $\mathrm{L}^{\mathrm{h}} / \mathrm{L}^{\mathrm{s}}$ through (Raj and Freed, 1992) 


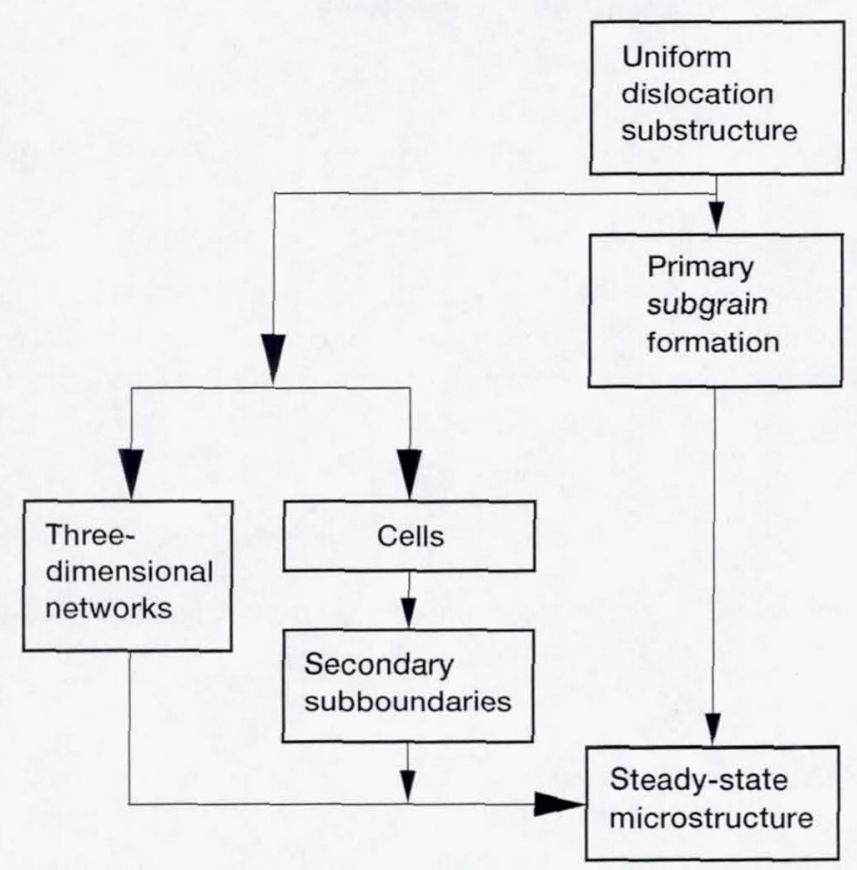

Figure 20.-Schematic showing the different creep substructures that may develop from a clustering of an initial uniform distribution of dislocations.

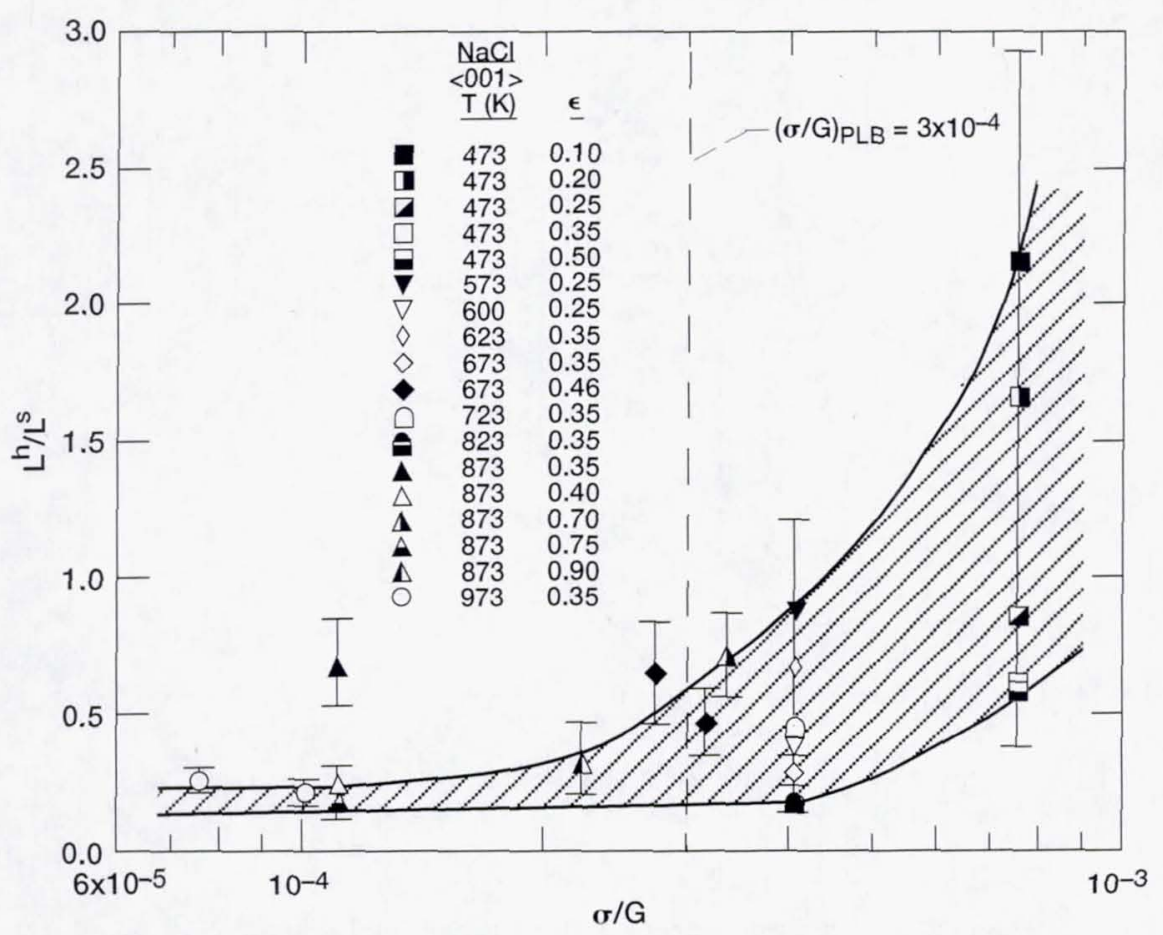

Figure 21.-Ratio of the average dimensions of the cell width to the cell interior versus normalized stress for sodium chloride single crystals deformed in the class $M$ and exponential creep regimes. This ratio tends towards a constant value of about 0.2 under conditions when steady-state creep is attained (Raj and Freed, 1992) (with kind permission from Elsevier Science Ltd.). 


$$
\mathrm{f}^{\mathrm{cb}}=1-\left[1 /\left(1+\mathrm{L}^{\mathrm{h}} / \mathrm{L}^{\mathrm{s}}\right)\right]^{3}
$$

a value of $\mathrm{L}^{\mathrm{h}} / \mathrm{L}^{\mathrm{s}}=0.2$ corresponds to $\mathrm{f}^{\mathrm{cb}}=0.42^{2}$.

The dislocation density within the subgrains is dependent on the normalized applied stress as

$$
\rho=(1 / \alpha \mathrm{Mb})^{2}(\sigma / \mathrm{G})^{2}
$$

where $0.1 \leq \alpha \leq 1$, although typically $\alpha \approx 0.5$ for $M=2$ (Fig. 22) (Bird et al., 1969; Takeuchi and Argon, 1976).

Equations (12) and (20) are similar in form when the terms are rearranged. In other words, equation (12) specifically applies when the obstacles to dislocation motion are other dislocations in the network within the primary subgrains. However, when cell boundaries are the obstacles, then equation (18)(b) suggests that $\sigma_{\text {obs }}$ is better defined as

$$
\sigma_{\mathrm{obs}} \approx 4 \times 10^{5} \mathrm{G}\left(\mathrm{b} / \mathrm{d}_{\mathrm{c}}\right)^{2}
$$

especially since the steady-state microstructures show that the cell interiors contain little or no dislocations (Figs. 13 and 15).

\subsection{Effect of Creep Strain on Substructure}

\subsubsection{Class M Creep Regime}

Although stress plays an important role in determining the nature, size and morphology of the substructure, strain and temperature can also influence the creep microstructure. An increase in both these parameters generally tends to favor cell and subgrain formation with a corresponding reduction in the dislocation density.

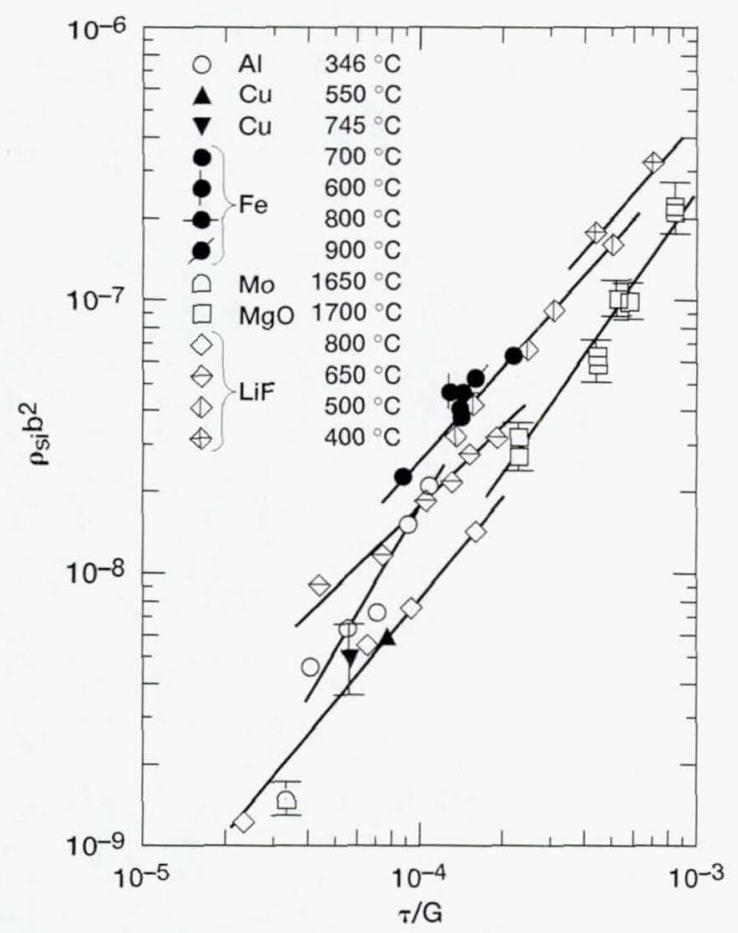

Figure 22.-Stress dependence of the dislocation density within the subgrain interiors in several crept materials (Takeuchi and Argon, 1976) (with kind permission from Chapman and Hall).

\footnotetext{
${ }^{2}$ Equation (19) is a general derivation of $\mathrm{f}^{\mathrm{cb}}$ in comparison to an earlier definition (Raj and Pharr, 1992) since it is independent of the geometry of the cell.
} 
It is fairly well established that the substructure morphology changes with creep strain for many materials, where most of these studies have been conducted in the class M creep region (Takeuchi and Argon, 1976). These investigations reveal a certain general trend in creep substructure formation, which has been summarized by Takeuchi and Argon (1976) (Fig. 23). First, a uniform dislocation substructure forms on loading. As creep progresses, recovery begins and the initial substructure transforms itself into a cellular or subgrain microstructure resembling that formed during stage III deformation at low temperature deformation (Fig. 23(a)). Additional straining results in further clustering of the dislocations into a heterogeneous substructure consisting of some regions with a high density of parallel dislocation walls and others with low dislocation density (Fig. 23(b)). Continued straining produces a banded microstructure of alternate regions of elongated and equiaxed subgrains (Fig. 23(c)). The elongated subgrains tend to become equiaxed as the banded microstructure transforms to a homogeneous distribution of equiaxed subgrains with a cellular or a three-dimensional network within them (Fig. 23(d)). Experimental observations by Hasegawa et al. (1971) on a Cu single crystal suggest that the local creep rate is higher in the equiaxed (i.e., coarse) than in the elongated (i.e. fine) subgrains (Fig. 24(a)). However, the local creep rates in both regions approach a common strain rate as the subgrain size attains a uniform value throughout the specimen (Fig. 24(b)).

Figure 25 shows the variation of the total dislocation density, $\rho_{\mathrm{T}}$, the dislocation density in the subboundaries, $\rho_{\mathrm{sb}}$, and that within the subgrains, $\rho_{\mathrm{si}}$, with creep strain (Orlová et al., 1972(a); Orlová and Č adek, 1973; Takeuchi and Argon, 1976), where $\rho_{\mathrm{T}}=\rho_{\mathrm{sb}}+\rho_{\mathrm{si}}$. The total dislocation density and the dislocation density within the sub-boundaries generally increase monotonically to steady-state values whereas $\rho_{\mathrm{si}}$ first increases steeply

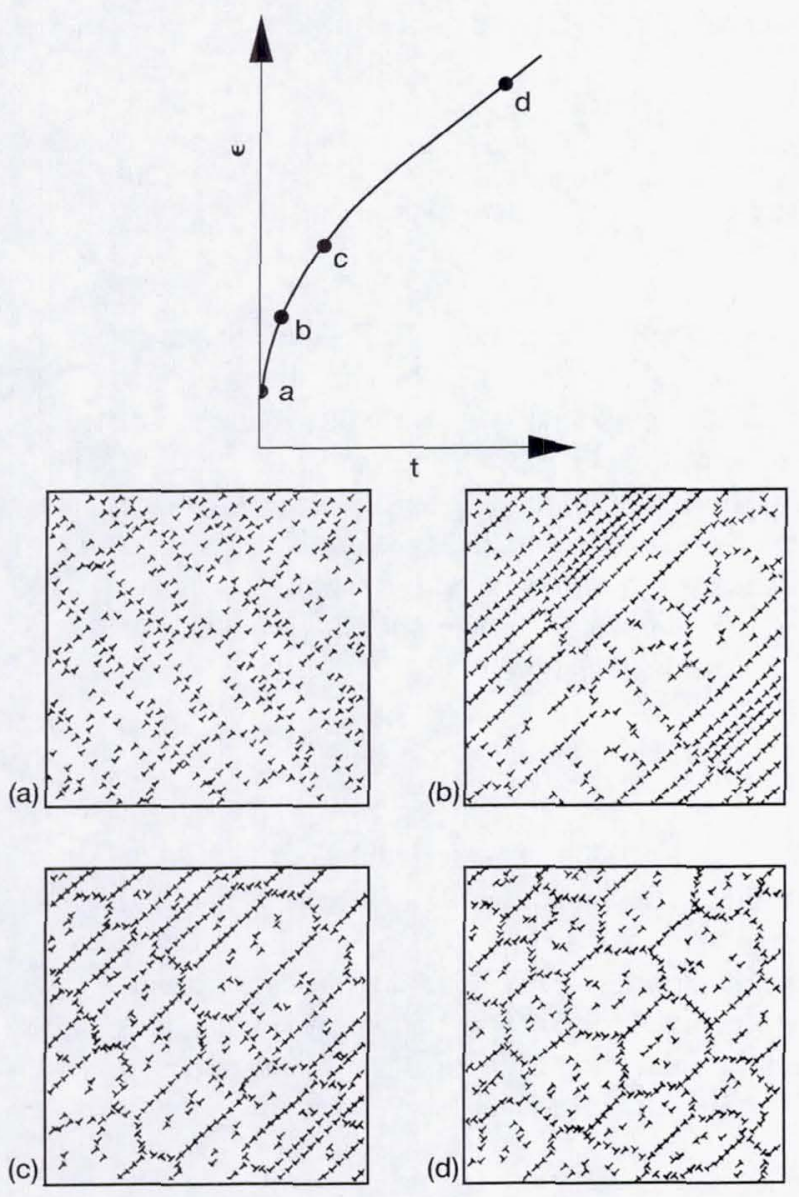

Figure 23.-Schematic showing the development of the creep substructure during class $M$ creep at different stages of the primary and secondary creep regions. (a) On loading; (b) early stages of primary creep; (c) later stage of primary creep; and (d) secondary creep (Takeuchi and Argon, 1976) (with kind permission from Chapman and Hall).
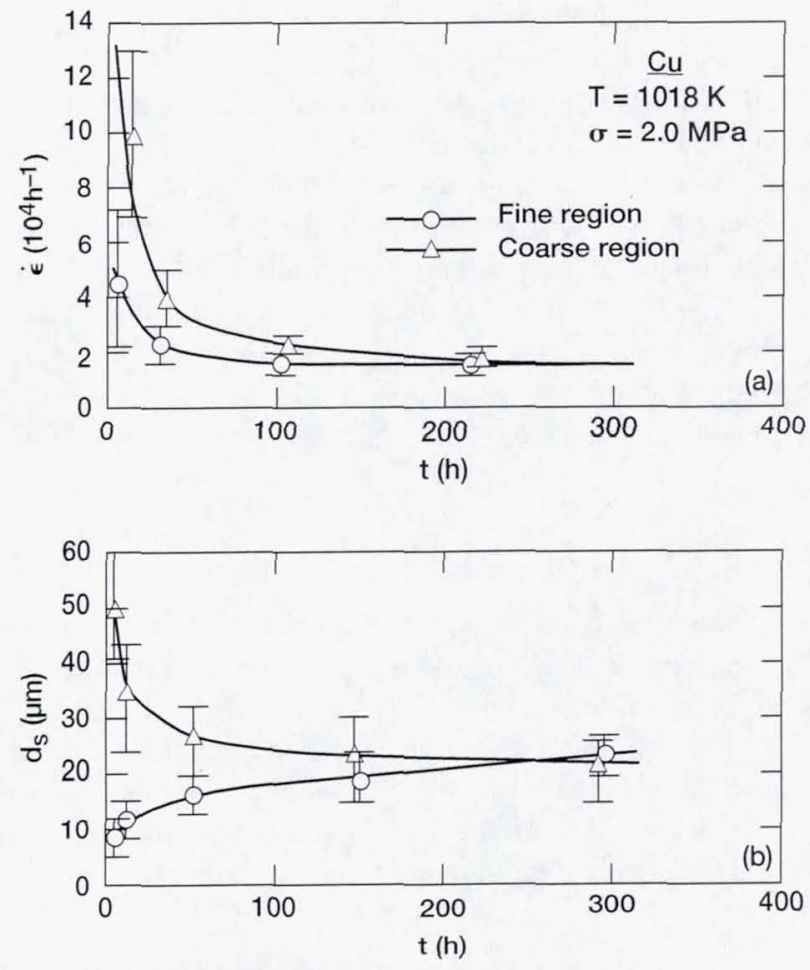

Figure 24.-Variations in the (a) local creep rate and (b) subgrain size for coarse and fine subgrains during primary and secondary stages of class $M$ creep of copper single crystals (Hasegawa et al., 1971; Takeuchi and Argon, 1976) (with kind permission from Chapman and Hall). 


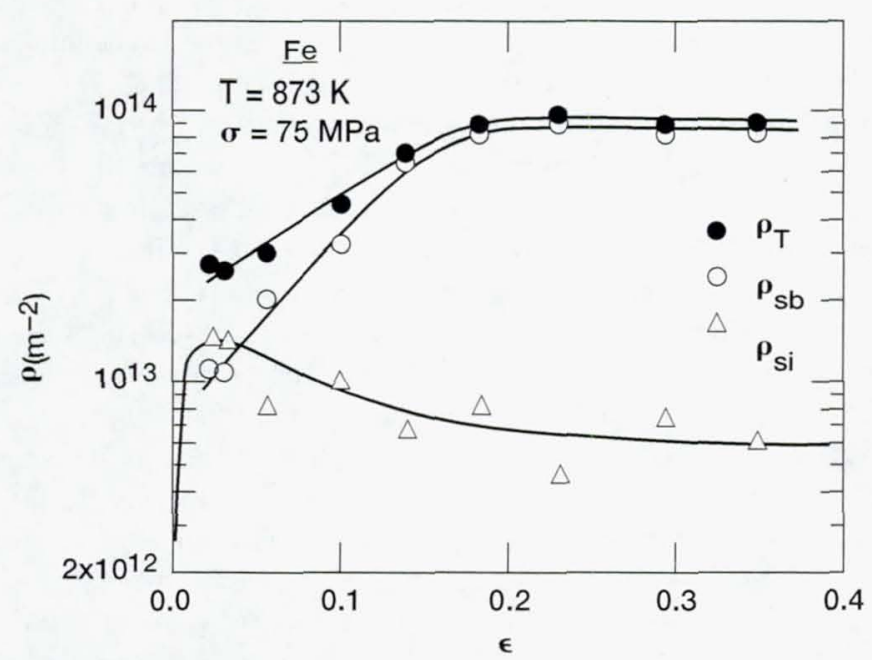

Figure 25.-Variations in the total dislocation density, and the dislocation densities in the subboundaries and within the subgrains with creep strain in $\alpha$-iron (Orlová et al., 1972; Orlová and Čadek, 1973; Takeuchi and Argon, 1976) (with kind permission from Chapman and Hall).

and then decreases to a constant value with increasing creep strain.

\subsubsection{Exponential Creep Regime}

A similar progressive variation in substructure with creep strain is observed in $\mathrm{NaCl}$ single crystals deformed at $473 \mathrm{~K}$ and $10.0 \mathrm{MPa}$ (i.e., $\sigma / \mathrm{G}=7.5 \times 10^{-4}$ ) in the exponential creep regime (Raj and Pharr, 1986(b)). The microstructure consists predominantly of a mixture of regions consisting of a fairly uniform distribution of dislocations interspersed with light patches and subboundaries after a creep strain of about 10 percent (Fig. 26(a)). Long straight subboundaries, oriented approximately parallel to the $\langle 100\rangle$ direction, form at $\varepsilon \approx 0.2$ (Fig. 16(d)). The first signs of the formation of rudimentary cells and subgrains become evident at $\varepsilon \approx 0.25$ as the initial dislocation substructure begins to recover (Fig. 26(b)). Continued deformation to $\varepsilon \approx 0.5$ results in the formation of elongated subgrains with higher dislocation density and equiaxed subgrains with lower dislocation density (Fig. 26(c)). Recent observations suggest that $\mathrm{L}^{\mathrm{h}} / \mathrm{L}$ decreases inversely with $\varepsilon$. The empirical relation

$$
\chi=\mathrm{L}^{\mathrm{h}} / \mathrm{L}^{\mathrm{s}} \approx 0.2(1+(1 / \varepsilon))
$$

was observed to fit the experimental data fairly well (Raj and Freed, 1992). The smallest value of $\varepsilon$ is $\varepsilon_{0}$ at the start of a creep test, where $\varepsilon_{0}$ is the instantaneous creep strain on loading. Thus, equation (22)(a) suggests that $\mathrm{L}^{\mathrm{h}} / \mathrm{L}^{2} \approx 4$ for a typical value of $\varepsilon_{0} \approx 0.05$ at $\mathrm{t}=0$.

However, an alternative mathematical fit derived from the viscoplasticity literature (Armstrong and Frederick, 1966) leads to a more attractive formulation for the functional form of $\chi$ in part because it contains hardening and dynamic softening terms and in part because it is more conducive to numerical analysis than equation (22a). Therefore, expressing the time derivative of $\chi$ as

$$
\dot{\chi}=-\mathrm{B}_{2}\left[\chi_{0}-\left(\mathrm{A}_{2} / \mathrm{B}_{2}\right)\right] \exp \left(-\mathrm{B}_{2} \varepsilon\right) \dot{\varepsilon}
$$

it can be shown that

$$
\chi=\left(\mathrm{A}_{2} / \mathrm{B}_{2}\right)+\left[\chi_{0}-\left(\mathrm{A}_{2} / \mathrm{B}_{2}\right)\right] \exp \left(-\mathrm{B}_{2} \varepsilon\right)
$$

where $\mathrm{A}_{2}$ and $\mathrm{B}_{2}$ are constants and $\chi_{0}$ is the initial value of $\chi$. The first term in the parenthesis in equation (22b) 

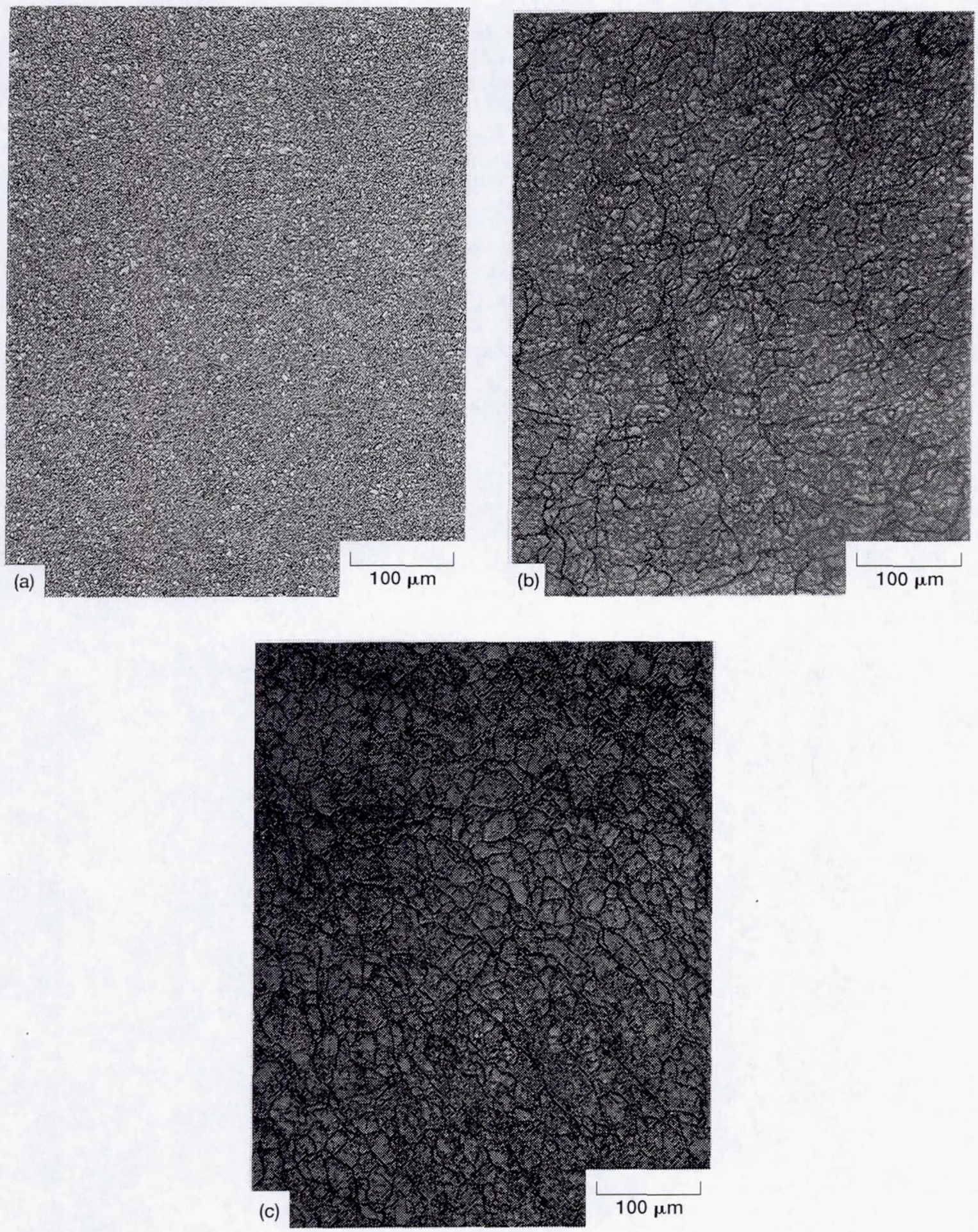

Figure 26.-Strain dependence of the creep substructure in the exponential creep region in sodium chloride single crystals crept at $473 \mathrm{~K}$ under a normalized stress of $7.5 \times 10^{-4}$. (a) $\epsilon=0.10 ;(b) \epsilon=0.25$; and (c) $\epsilon=0.50$. (Raj and Pharr, 1986 (b)). 
represents the hardening coefficient while the second term is the softening component. The ratio, $A_{2} / B_{2}$, represents the steady-state value of $\chi$ so that from Fig. $21, \mathrm{~A}_{2} / \mathrm{B}_{2} \approx 0.2$. Equation (22c) fits the experimental data fairly well for values of $\mathrm{A}_{2} / \mathrm{B}_{2}=0.2, \chi_{0}=4.0$ and $\mathrm{B}_{2}=5.4$ (Fig. 27). The value of $\chi_{0}=4.0$ corresponds to an initial value of $\mathrm{f}^{\mathrm{cb}}=0.99^{3}$.

The above description of the creep microstructure reveals two important results. First, there are large similarities between substructure formation during the transient stages of the class M creep regime (Fig. 23) (Takeuchi and Argon) and that existing in the exponential creep region (Fig. 26). In other words, these observations suggest that the constitutive law for primary creep is the exponential creep law which reduces to a power-law relation in the limit of steady-state deformation. This is shown schematically in Fig. 28 for constant strain rate, constant stress and constant load conditions involving compression and tension tests. Figure 28(a) shows the different stages A, B, C and $\mathrm{D}$ in the primary and secondary creep regions. The structure parameters, $\mathrm{S}_{\mathrm{i}}$, corresponding to the points A, B, C and $\mathrm{D}$ are also marked on the creep curve. The thin curves in Fig. 28(b) represent the constitutive $\dot{\varepsilon} \exp \left(\mathrm{Q}_{\mathrm{c}} / \mathrm{RT}\right)-$ $\sigma / \mathrm{G}$ relation, for a constant value of the structure parameter. The bold curve in Fig. 28(b) represents the experimentally-observed class $\mathrm{M}$ and exponential creep relations with $\mathrm{n}>3$. The transition points $\mathrm{A}, \mathrm{B}, \mathrm{C}$ and $\mathrm{D}$ shown in Fig. 28(a) are also marked in Fig. 28(b). Thus, based on the microstructural evidence presented earlier, primary creep can be interpreted as transition stages along several constant structure exponential creep curves. Although the same steady-state point, D, can be reached either by constant strain rate or constant stress deformation techniques (Weertman, 1956), Fig. 28(b) reveals that the development of the steady-state microstructures does not follow the same thermodynamic path.

An outcome from Fig. 28 is a rationalization for the deviations of the experimental values of the stress exponent from the universal creep law. The broken line with $n=3$ shown in Fig. 28 represents the universal creep law predicted by all climb-controlled models (Weertman, 1975). These models ignore contributions from other mechanisms so that this line is a limiting case. Therefore, based on Fig. 28 , the higher values of $n \approx 4.5$ observed for many materials (Bird et al., 1969) suggest that the contributions from nondiffusional processes are sufficient to increase $n$ from 3 to 4.5 . Additionally, the experimental values of $\mathrm{V}^{*}$ can be explained by including contributions from nondiffusional mechanisms. If class $M$ behavior is solely due to dislocation climb, then theory predicts that $\mathrm{V}^{*} \approx 1 \mathrm{~b}^{3}$ (Conrad, 1964; Evans and Rawlings, 1969). However, in actuality, $\mathrm{V}^{*} \approx 5 \times 10^{2}$ to $10^{4} \mathrm{~b}^{3}$ for many materials in the class M creep region (Balasubramanian and Li, 1970; Raj and Pharr, 1986(b); and Raj, 1989), which is several

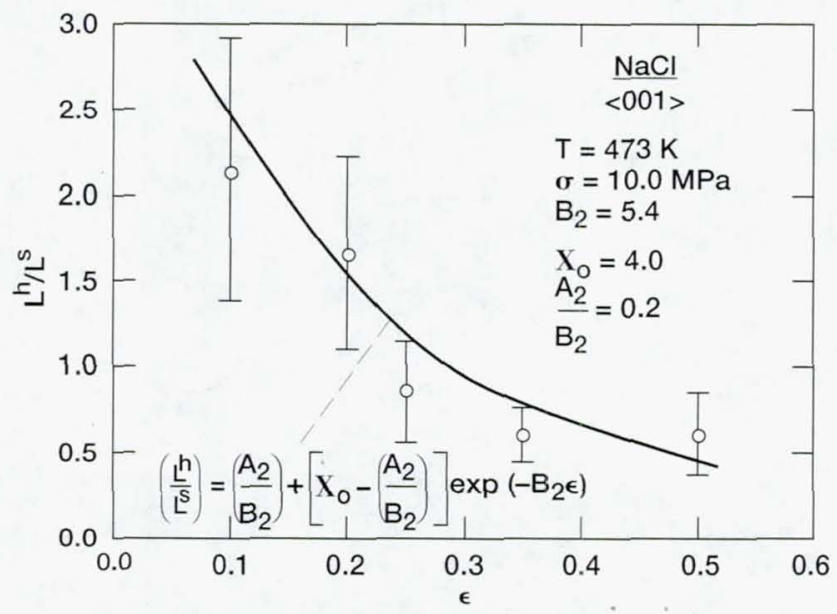

Figure 27.-Changes in the ratio of the average dimensions of the cell width to the cell interior with creep strain in the exponential creep region in sodium chloride single crystals crept at $473 \mathrm{~K}$ under a normalized stress of $7.5 \times 10^{-4}$ (data from Raj and Freed, 1992).

\footnotetext{
${ }^{3}$ It should be noted that cells have not formed during this early stage of deformation. Therefore, $\mathrm{f}^{\mathrm{cb}}$ has no physical meaning in terms of describing the volume fraction of the cell boundaries. Instead, a high value of $f^{\mathrm{cb}}=0.99$ signifies that there is a homogeneous distribution of dislocations formed in the material soon after loading.
} 

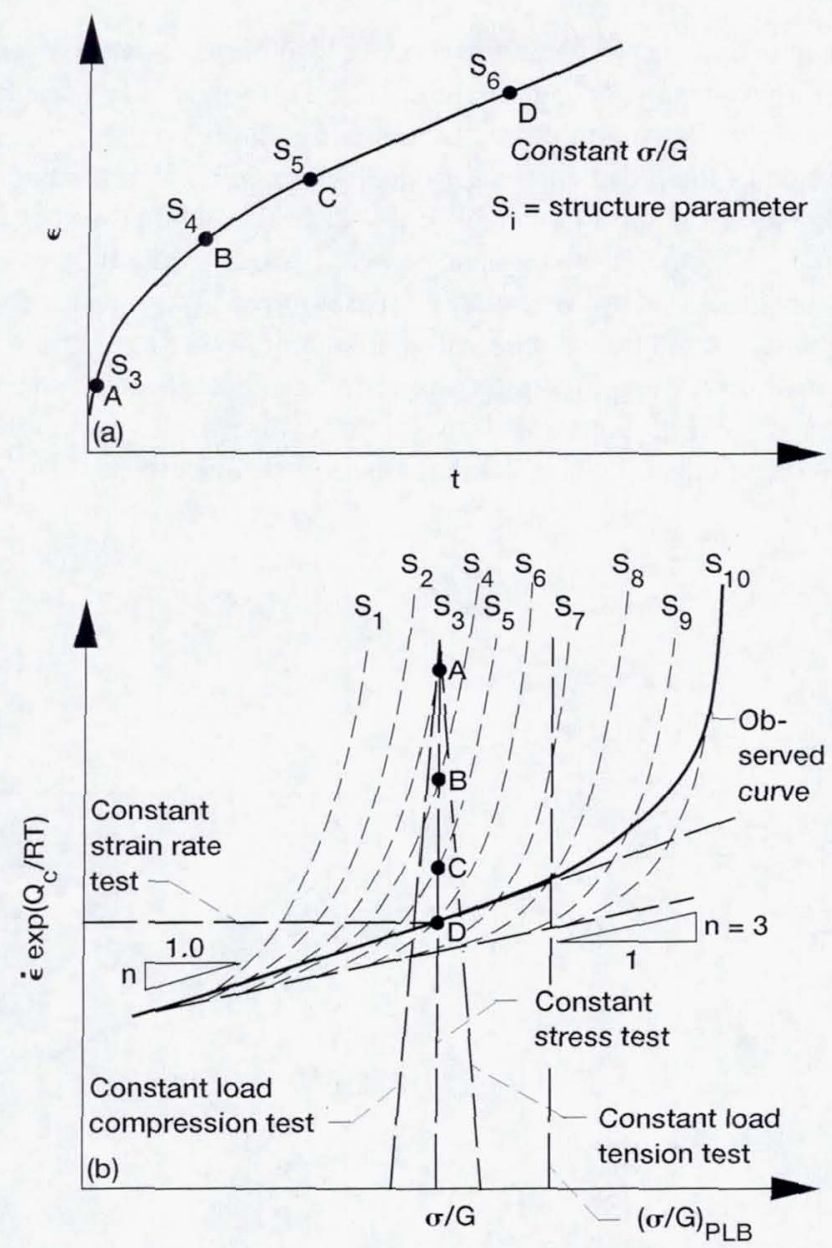

Figure 28.-Schematic plot demonstrating that the transient primary creep stages, $A, B$, and C of the creep curve in (a) correspond to the structure-dependent (i. e. $S_{i}$ ) exponential curves in the normalized creep rate-normalized stress relationship shown in (b); $D$ is at or close to the steady-state point. The deformation paths followed in constant strain rate, constant stress and constant load tests are indicated in (b). The broken line with $n=3$ represents the natural creep law.

orders of magnitude higher than that expected for dislocation climb. The implication of these observations is that experimental values of $\mathrm{V}^{*}>\mathrm{b}^{3}$ cannot be rationalized if contributions from nondiffusional creep mechanisms are ignored in the class $\mathrm{M}$ region.

Second, Figs. 26(a) to (c) reveal that a homogeneous dislocation microstructure is essentially unstable during deformation and it tends to transform to a heterogeneous substructure comprising of 'hard' regions with high dislocation densities (e.g., elongated subgrains, and cell and subgrain boundaries) and "soft" regions with low dislocation densities (e.g., equiaxed subgrains, and cell and subgrain interiors). These observations imply that the oneparameter modeling approach based solely on the stress dependence of the dislocation density is incomplete (Mecking and Kocks, 1981; Estrin and Mecking, 1984). Nix et al. (1985(a) and (b)) proposed using a two-parameter approach based on subgrain interior ('soft' regions) and subboundaries ('hard' regions), but in view of the actual nature of the deformation microstructures, even this method is unrealistic. In this regard, the DSV model proposed by Freed et al., (1992) provides a more realistic framework for developing a microstructural-based creep model.

\subsection{Effect of Temperature on Substructure}


Relatively few creep data exist in the literature where creep curves have been generated at a single value of normalized applied stress over a wide range of temperatures. Such information is useful not only in evaluating the specific form of equation (1) but also in isolating the effect of temperature on the development of the creep substructure. Figure 29 shows an example of the creep curves for $\mathrm{NaCl}$ generated over a temperature range of 573 to $873 \mathrm{~K}$ at a nominal value of $\sigma / G=4 \times 10^{-4}$ (Raj and Pharr, 1992). It is seen that while the creep rates decrease monotonically with increasing strain, there is an increased tendency towards steady-state behavior with increasing temperature. Detailed microstructural observations revealed that temperature variations affect the microstructure in a subtle but significant manner, where microstructural refinement is greater at higher temperatures (Raj and Pharr, 1992). The net effect of this increased tendency towards microstructural refinement with increasing temperature results in a decrease in $\mathrm{L}^{\mathrm{h}} / \mathrm{L}^{\mathrm{s}}$ (Fig. 30(a)) and $\mathrm{f}^{\mathrm{cb}}$ (Fig. 30(b)) with increasing temperature for the cells and cell boundaries. Similar results are expected to be valid for the subgrains. These temperature dependencies are given by the regression equations

$$
\chi=\mathrm{L}^{\mathrm{h}} / \mathrm{L}^{\mathrm{s}}=13.8 \exp (-0.005 \mathrm{~T})
$$

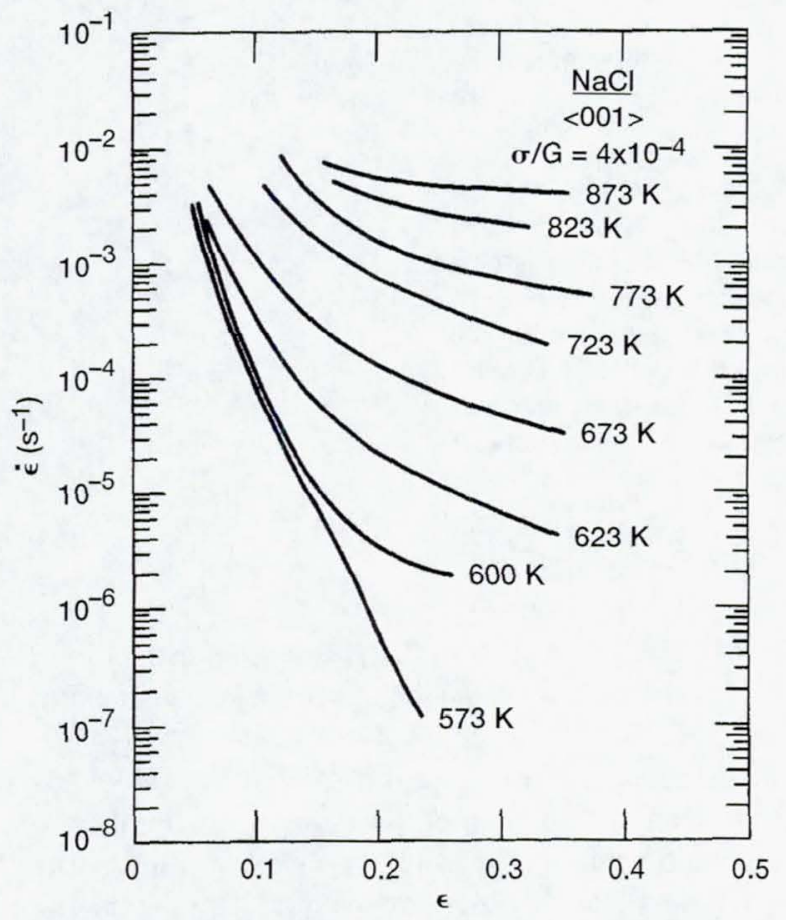

Figure 29.-Plot of creep rate versus true strain for $\mathrm{NaCl}$ single crystals deformed between 573 and $873 \mathrm{~K}$ under a normalized stress of about $4 \times 10^{-4}$ (Raj and Pharr, 1992) (reprinted by permission of the American Ceramic Society).
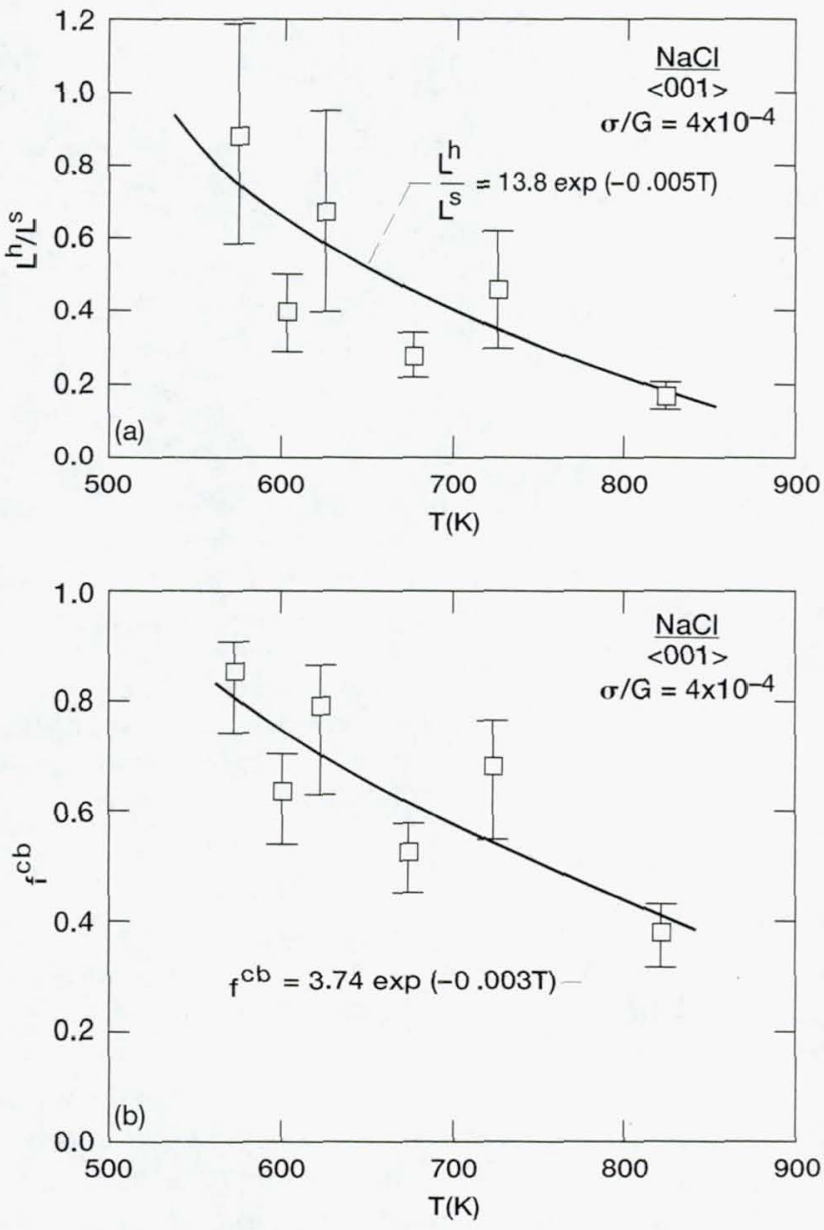

Figure 30.-Changes in (a) the ratio of the average dimensions of the cell width to the cell interior and (b) volume fraction of the cell boundaries with absolute temperature in sodium chloride single crystals crept in the class $M$ and exponential creep regimes under a normalized stress of $4.0 \times 10^{-4}$ (data from Raj and Pharr, 1992). 


$$
\mathrm{f}^{\mathrm{cb}}=3.74 \exp (-0.003 \mathrm{~T})
$$

As detailed in $\S 3.1$, the stress dependence of the substructure, especially its effect on the subgrain size, is well characterized for a number of materials (Bird et al., 1969; Takeuchi and Argon, 1976; Thompson, 1977;

Bendersky et al., 1985; Orlová and Cadek, 1986; Raj and Pharr, 1986). In contrast, there is relatively little information available on the effect of temperature on the cell or subgrain size. Early investigations on the subject suggested that temperature affects both the morphology and dimenșions of the substructure (Myshlyaev, 1967; Orlová et al., 1972; Belkin et al., 1973; Michel et al., 1973; Streb and Reppich, 1973; Kestenbach et al., 1976, 1978). For example, it has been suggested that the substructure changes from a configuration of dislocation tangles and cells to well-formed subgrains with increasing temperature (Michel et al., 1973; Kestenbach et al., 1976, 1978). Although an increase in $d_{c}$ or $d_{s}$ with increasing temperature was reported in some instances (Myshlyaev, 1967; Orlová et al., 1972(b); Belkin et al., 1973; Michel et al., 1973; Streb and Reppich, 1973), other observations suggest that $d_{c}$ and $d_{s}$ are relatively independent of temperature (Orlová et al., 1972; Young and Sherby, 1973; Blum, et al., 1980). However, there is little direct evidence to confirm this lack of temperature dependence of the cell and subgrain size since this conclusion has been drawn primarily from studies where the effects of temperature were of secondary importance to the effects of stress. For example, plots of $\mathrm{d}_{\mathrm{s}} / \mathrm{b}$ against $\sigma / \mathrm{G}$ show the data are usually clustered around the mean line, thereby suggesting the effects of temperature are small (Raj and Pharr, 1986(a)). Although these indirect observations rule out strong temperature dependencies, they do not permit the separation of weak temperaturedependent variations in $d_{c}$ and $d_{s}$ from experimental scatter.

These small temperature effects, if present, can be better identified from direct measurements of $d_{c}$ and $d_{s}$ as a function of temperature at constant values of $\sigma / \mathrm{G}$. Unfortunately, the number of investigations in which such direct measurements have been made are small and the results ambiguous. Direct measurements of $d_{s}$ as a function of temperature at constant values of $\sigma$ (Orlová et al., 1972; Blum et al., 1980) revealed that $\mathrm{d}_{\mathrm{s}}$ is essentially independent of temperature within experimental scatter. Recent results on $\mathrm{NaCl}$ suggest that $\mathrm{d}_{\mathrm{s}}$ increases by a factor of about two with increasing temperature while $\mathrm{d}_{\mathrm{c}}$ is almost independent of temperature for a variation in temperature by $300 \mathrm{~K}$ (Fig. 31) (Raj and Pharr, 1992). Since errors in measuring $d_{c}$ and $d_{s}$ are within a factor of two, the small temperature dependence of $d_{s}$ shown in Fig. 31 cannot be considered to be significant.

The limited direct measurements of the temperature dependence of the cell and subgrain sizes suggest that the final dimensions of the creep substructure are influenced primarily by the applied stress, and strain, and only secondarily by temperature. However, since diffusion-controlled recovery mechanisms, such as dislocation climb, become significant at high temperatures, the kinetics of formation of the steady-state substructure increases with increasing temperature. As a result, excess dislocations are annihilated and the width of the cell and subgrain walls decreases with a corresponding increase in the dimensions of the cell interior (Fig. 30(a)).

\subsection{Internal Stresses Associated with the Formation of Substructure}

The concept of a long range internal back stress has a sound thermodynamic basis in the theory of deformation (Hirth and Nix, 1969; Kocks et al., 1975; Gibeling and Nix, 1980; Nadgornyi, 1988). There is now considerable experimental evidence which suggests that the evolution of a steady-state creep substructure leads to a corresponding build-up of the internal stresses within the material. This in turn results in a decrease in the creep rate during primary creep to a constant value during steady-state creep. This close correlation between creep substructure and $\sigma_{\mathrm{b}}$ is clearly revealed in Fig. 32 in the classic study by Hasegawa et al., (1972) on a Cu-16(at.\%) Al alloy. This alloy exhibits sigmoidal creep, where the creep rate first increases due to a corresponding rise in the total dislocation density and then decreases to a steady-state value with the advent of cell formation. The microstructure during the initial period of creep consists of isolated dislocations, and $\sigma_{\mathrm{b}} / \sigma \approx 0.3$ remains virtually unchanged during this time. As creep progresses, the isolated dislocations cluster themselves to form cells so that the dislocation density within the cells decreases with creep time or creep strain. Thus, cell formation is associated with a steep increase in $\sigma_{b} / \sigma$ and a corresponding decrease in the creep rate. The development of internal stresses within the material due to cell or subgrain formation is not unique to creep. The build-up of internal stresses can occur under other deformation modes, such as fatigue and uniaxial constant strain rate deformation. For example, Fig. 33(a) shows the distribution of the average global and local shear stresses present in the channels and walls of persistent slip bands in a fatigued copper specimen, where these stresses were determined from measurements of the radii of curvature of bowed out dislocations (Mughrabi, 1983). Figure 33(b) is a schematic of the idealized illustration of this stress distribution 


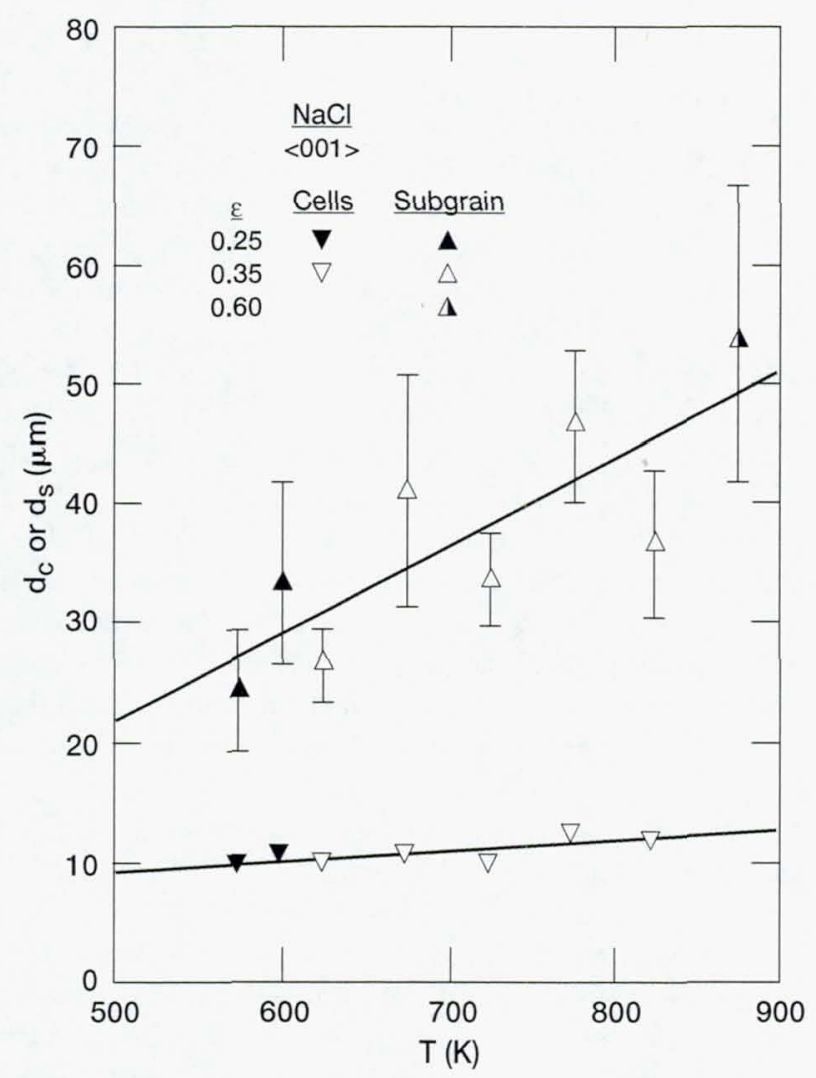

Figure 31.-Plot of the cell and subgrain size against absolute temperature for $\mathrm{NaCl}$ single crystals deformed under a normalized stress of about $4.0 \times 10^{-4}$ (Raj and Pharr, 1992) (reprinted by permission of the American Ceramic Society).

showing the relative magnitudes of the local tensorial back, B, and forward, F, stresses with respect to the tensorial global stress, $\sigma^{4}$. Indirect evidence for the increase in $\sigma_{\mathrm{b}}$ with cell or subgrain formation is reflected in the decrease in $\mathrm{L}^{\mathrm{h}} / \mathrm{L}^{\mathrm{s}}$ with increasing $\varepsilon$ (Fig. 27).

The existence of an internal back stress acting on the dislocation within a cell or subgrain interior due to the presence of the cell or subgrain boundaries suggests that the local effective stress, $\sigma_{e}(x, t)=\sigma-\sigma_{b}(x, t)$, where the spatial and time dependence of $\sigma_{\mathrm{e}}$ and $\sigma_{\mathrm{b}}$ arise due to the dynamic nature of the substructure. Thus, the average creep rate can be expressed as $\dot{\varepsilon} \alpha\left(\Sigma^{\mathrm{N}} \sigma_{\mathrm{e}}(\mathrm{x}, \mathrm{t}) / \mathrm{N}\right)^{\mathrm{n}^{\prime}} \alpha\left(\sigma-\Sigma^{\mathrm{N}} \sigma_{\mathrm{b}}(\mathrm{x}, \mathrm{t}) / \mathrm{N}\right)^{\mathrm{n}^{\prime}}$, where $\mathrm{N}$ is the number of cell or subgrain boundaries and $n$ ' is the effective stress exponent. Since $\sigma_{b}$ develops in response to microstructural changes occurring under the action of an applied stress during the course of an uninterrupted creep test, the latter type of experiments do not permit an effective separation of $\sigma_{\mathrm{b}}$ from $\sigma$. However, it is clear that $\sigma_{\mathrm{e}}<0$, and hence $\varepsilon<0$, when $\sigma<$ $\sigma_{\mathrm{b}}$. Therefore, reducing the applied stress from $\sigma \geq \sigma_{\mathrm{b}}$ to $\sigma<\sigma_{\mathrm{b}}$ should lead to a decoupling $\sigma_{\mathrm{b}}$ from $\sigma$, and therefore provide a method for measuring $\sigma_{b}$.

The magnitude of $\sigma_{b}$ is determined experimentally from either stress decrease (or "stress dip") or stress relaxation tests. Several methods based on one or the other technique have been reported in the literature (e.g., Davies et al., 1973; Gibeling and Nix, 1977; Blum and Finkel, 1982; Pahutová et al. 1977, 1993; Yamada and Li, 1975; Toma et al., 1976; Yoshinaga, 1993), but the interpretation of the results has been controversial and confusing primarily since it is not always clear which specific microstructural event (e.g., dislocation bowing, grain boundary

\footnotetext{
${ }^{4}$ Note: The nomenclature B, F, $\sigma$ and $\tau$ has been used in order to be consistent with the tensorial representations described in section 6.2 . The subscripts have been omitted for the unidirectional stresses so that $\mathrm{B}=\sigma_{\mathrm{b}}$ and $\mathrm{F}=\sigma_{\mathrm{f}}$ describe the special case of unidirectional local back and forward stresses, respectively.
} 

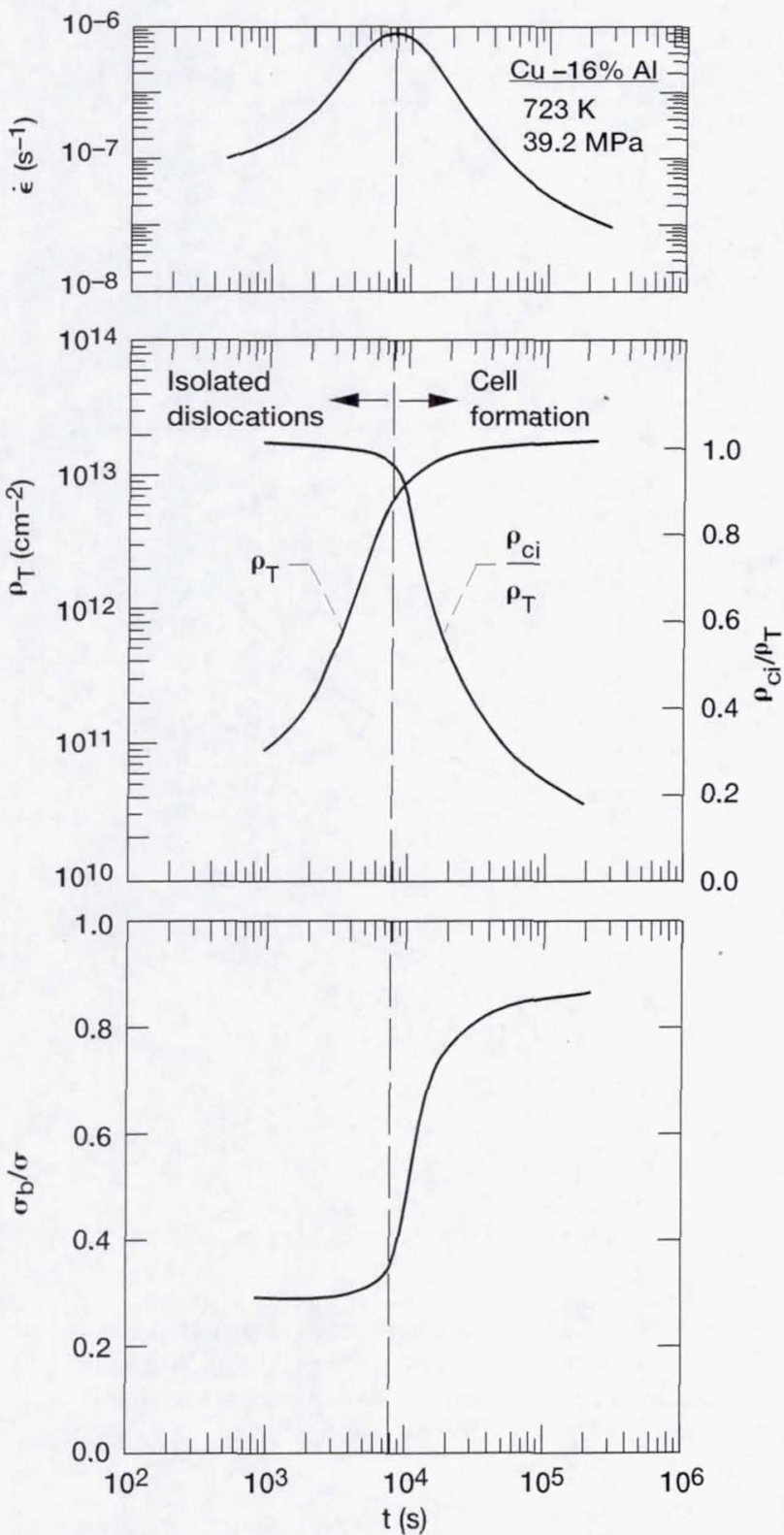

Figure 32.-Effect of cell formation on the creep rate and internal back stress in Cu-16\%Al (Hasegawa et al., 1972).
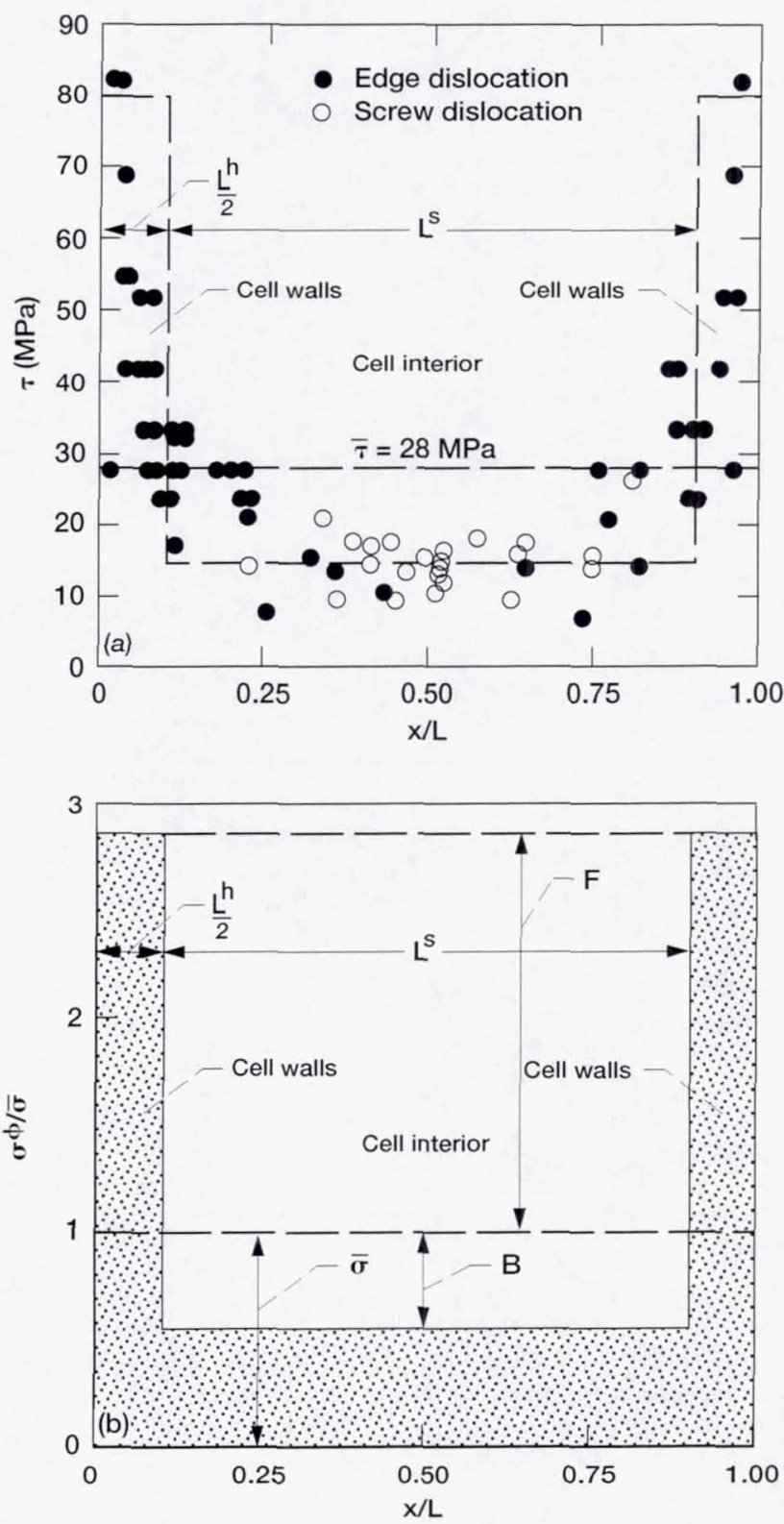

Figure 33.-Spatial distribution of the effective internal stresses acting in the cell interior (B) and on the cell boundary $(F)$, respectively. (a) Experimental data measured in copper in the persistant slip bands (data from Mughrabi, 1983); (b) schematic representation of the data in (a).

relaxation, dislocation run-off into the cell or subgrain boundaries, and subboundary migration) should be identified with $\sigma_{\mathrm{b}}$. Significantly, anelastic recovery strain associated with negative creep rates when $\sigma<\sigma_{\mathrm{b}}$ has been observed in single crystals where grain boundary effects can be ruled out as the primary cause of $\sigma_{\mathrm{b}}$ (Fig. 34) (Gibeling and Nix, 1981). These observations also confirm that back stresses arise as a consequence of the heterogeneous nature of the creep substructure (Nix and Ilschner, 1980).

Recent calculations also suggest that the local effective stresses acting in the cell interior, $\sigma_{\text {ci }}$, and on the cell boundaries, $\sigma_{\mathrm{cb}}$, change with increasing temperature as the dislocation substructure becomes increasingly heterogeneous (Fig. 35) (Raj and Pharr, 1992). Assuming a particular form for the temperature dependence of $\sigma_{b}$, these calculations show that as the temperature increases, there is a steep increase in the forward stress, $\sigma_{\mathrm{f}}$, acting on the cell boundaries which also results in a corresponding increase in $\sigma_{\mathrm{cb}}\left(=\sigma+\sigma_{\mathrm{f}}\right)$ and a decrease in $\sigma_{\mathrm{ci}}\left(=\sigma-\sigma_{\mathrm{b}}\right)$. 


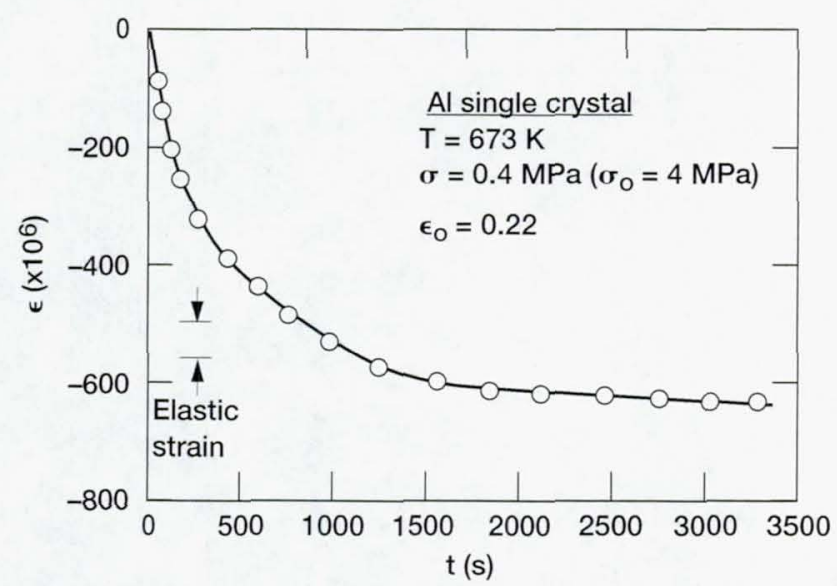

Figure 34.-Anelastic creep strain observed in an Al single crystal deformed to a strain of $\epsilon \approx 0.22$ at $673 \mathrm{~K}$ under an initial stress of $4 \mathrm{MPa}$ after the stress was reduced to $0.4 \mathrm{MPa}$ (Gibeling, 1979 cited by Nix and Ilschner, 1980).
$\mathrm{T}(\mathrm{K})$
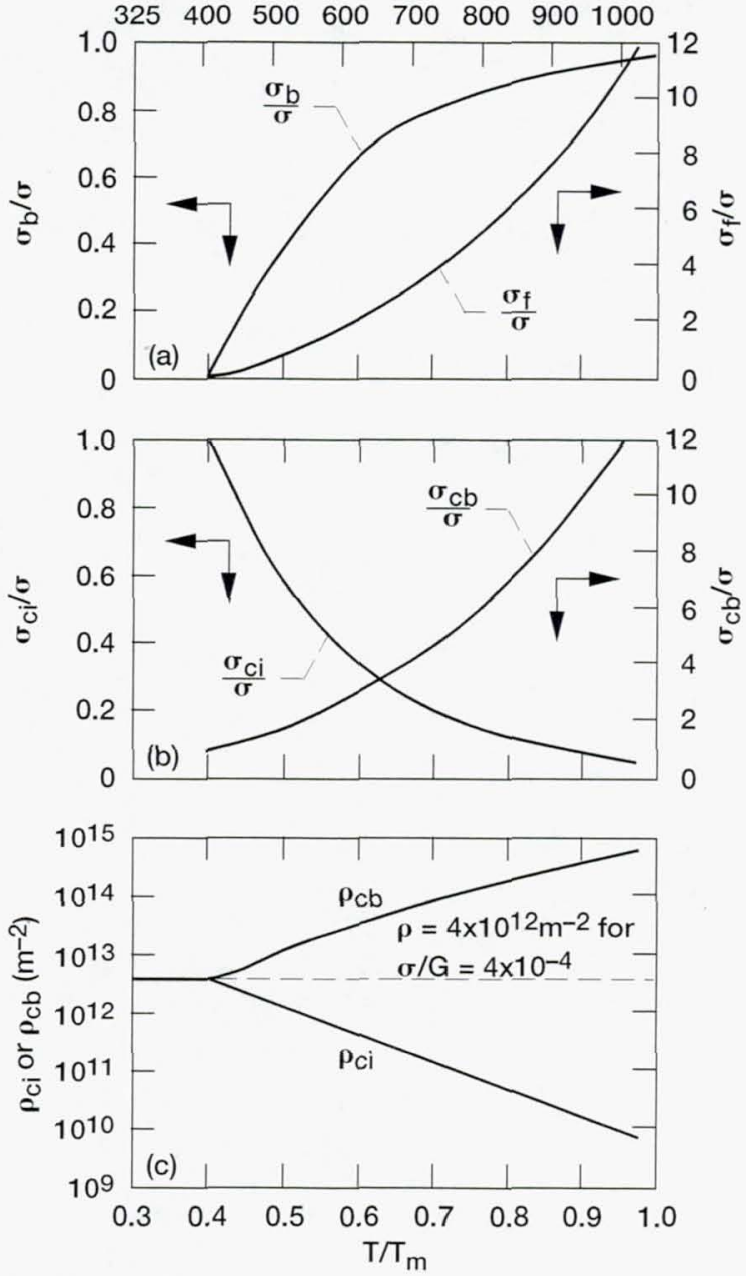

Figure 35.-Calculated values of the internal stresses and dislocation densities in the cell boundaries and cell interiors as a function of temperature (Raj and Pharr, 1992) (reprinted by permission of the American Ceramic Society).

These changes in the effective stresses arise due to a decrease in the dislocation density within the cells, $\rho_{\text {ci }}$, and increase in that in the cell boundaries, $\rho_{c b}$, with increasing temperature. Similar variations are expected for subboundaries.

\subsection{MICROSTRUCTURAL STABILITY}

Although the microstructural observations reported in $\$ 3$ provide important information on the qualitative and quantitative aspects of creep substructure, they do not address the problem of microstructural stability involving the morphological and dimensional variations in the substructure when the testing conditions are changed. Investigations on microstructural stability generally involve producing an initial microstructure which is then subjected to a different set of deformation conditions to understand how it transforms to the final substructure.

There has been a considerable amount of discussion in the literature (Poirier, 1972; Robinson et al., 1974; Pontikis and Poirier, 1975; Parker and Wilshire, 1976; Miller et al., 1977; Sherby et al., 1977; Ferreira and Stang, 1979, 1983; Blum et al., 1980; Langdon et al., 1980; Eggeler and Blum, 1981; Goel et al., 1983; Soliman et al., 1983; Ginter et al., 1984; Mohamed et al., 1985; Raj et al., 1989) regarding the stability of subgrains after a stress 
reduction. Equation (16) implies that the subgrain size should increase if the stress is decreased. However, several experimental observations have resulted in contradictory results. The first set of experiments conducted on $\mathrm{NaCl}$ (Poirier, 1972), $\mathrm{AgCl}$ (Pontikis and Poirier, 1975), $\mathrm{Cu}$ (Parker and Wilshire, 1976) and Al-5\% Zn (Langdon et al., 1979) revealed that the subgrains formed at the higher stress are relatively stable after a stress reduction and do not increase to a size consistent with the reduced stress. These experiments appeared to question the universality of equation (16). Miller et al. (1977) criticized some of these early observations and pointed out that the creep strain after the stress reduction was insufficient to permit the necessary amount of subgrain coarsening to allow the subgrain size to attain its equilibrium value at the lower stress. Langdon et al., (1980) addressed this criticism by measuring the subgrain size just after steady-state creep was re-established, but failed to observe any change in the subgrain size.

The second set of experimental observations on Al (Ferreira and Stang, 1979, 1980; Soliman et al., 1983; Mohamed et al., 1985), Al-5\% Zn (Blum et al., 1980; Goel et al., 1983) and $\mathrm{NaCl}$ (Eggeler and Blum, 1981) clearly demonstrated that substantial subgrain coarsening occurs after a stress reduction with a corresponding increase in the creep rate. The new subgrain size, similar to that predicted by equation (18) for the reduced stress, was attained typically when the creep strain after the stress reduction was greater than 3 percent (Raj et al., 1989). It was observed that subgrain coarsening after a stress reduction involves both the dissolution of some subboundaries and the migration of others (Eggeler and Blum, 1981; Goel et al., 1983; Soliman et al., 1983; Ginter et al., 1984; Mohamed et al., 1985). Soliman et al. (1983) suggested that Langdon et al. (1980) may have erroneously measured the cell size rather than the subgrain size which could account for the discrepancy in the two sets of observations.

The above stress change experiments have largely been conducted in the class M regime. However, they do not address two interesting and related questions regarding microstructural stability and their relation to the constitutive creep law. First, is the path along the class $\mathrm{M}$ to exponential creep plot, such as those shown in Figs. 9 and 10, reversible? Second, is the microstructure formed in the exponential creep region stable in the class $\mathrm{M}$ regime and vice versa? The experimental observations from experiments designed to address these questions are discussed below.

\subsection{Effect of Prior Deformation on Creep Substructure}

Two types of experiments were conducted on $\mathrm{NaCl}$ single crystals in order to study the path dependence of the constitutive equations for creep and microstructural stability (Raj et al., 1989). In the first series of experiments a specimen was deformed in the exponential creep region (Fig. 10). The specimen was cleaved parallel to the stress axis, and both halves were polished and etched. The substructure after creep under these conditions consisted of an uniform distribution of etch pits and a few ill-formed subboundaries similar to that shown in Fig. 16(d). One half of the cleaved specimen was then deformed further to a strain of $\varepsilon \approx 0.43$ at $873 \mathrm{~K}$ and at a stress of $1.0 \mathrm{MPa}$ (i.e., $\sigma / \mathrm{G}=1.1 \times 10^{-4}$ ) corresponding to the class $\mathrm{M}$ creep region (Fig. 10). The creep curve obtained under these conditions is shown in Fig. 36 along with that for an as-received specimen. In order to ascertain the effect of static recovery on the initial prestrained microstructure, the other half of the cleaved specimen was also placed in the creep machine close to the test specimen in such a manner that there was no applied stress on it.

The initial creep rate of the prestrained specimen was less than $10^{-8} \mathrm{~s}^{-1}$, which was below the detection limit of the strain-measuring equipment; measurable creep was detectable only after a period of $1 \mathrm{~h}$. However, the creep rate rises very rapidly to about $2 \times 10^{-5} \mathrm{~s}^{-1}$ within the first few percent strain before decreasing gradually to values close to that observed for the as-received specimen when $\varepsilon>0.3$ (Fig. 36).

The microstructure of the prestrained specimen after subsequent creep to a true strain of about 0.43 is shown in Fig. 37(a), where it is seen that the initial substructure (Fig. 16(d)) has transformed to a microstructure of well developed primary subgrains, secondary subboundaries and cells. This microstructure is similar to that observed on an as-received specimen deformed to an almost identical value of strain (Fig. 37(b)). The cell and subgrain sizes of the prestrained and the as-received materials are in agreement to within a factor of two which is the normal level of scatter associated with these measurements (Table III). For comparison, the predicted values of the cell and subgrain sizes from equations (15a) and (b) are $\mathrm{d}_{\mathrm{c}} \approx 22.5$ and $\mathrm{d}_{\mathrm{s}} \approx 135 \mu \mathrm{m}$, respectively.

Figure 37(c) shows the effect of static annealing on the initial predeformed microstructure (Fig. 16 (d)). Although the extent of recovery due to static annealing is not as extensive as that during deformation, it is not negligible, and as shown in Fig. 37(c), a few large subgrains, subdivided by cells, have already formed during this period. A comparison of Figs. 37(a) and (c) clearly demonstrates that stress-assisted recovery is much faster than static recovery. 


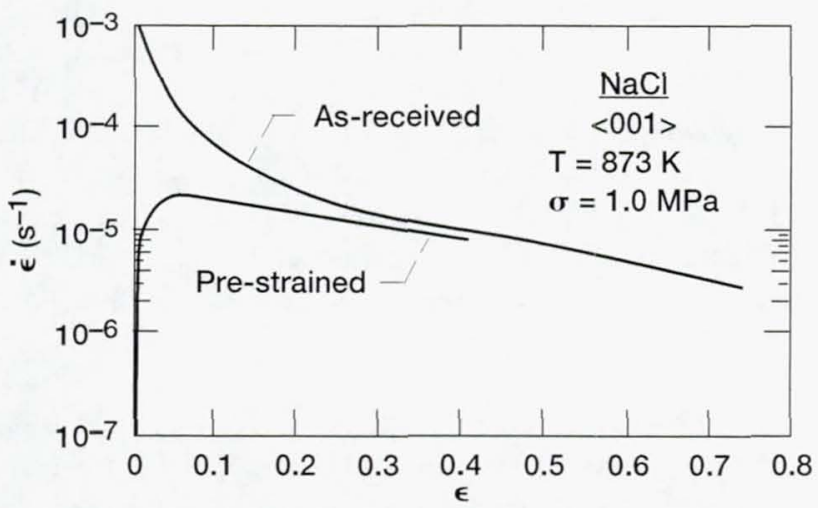

Figure 36.-Comparison of the creep curves for asreceived and prestrained sodium chloride single crystal specimens deformed at $873 \mathrm{~K}$ under a normalized stress of $1.1 \times 10^{-4}$. The prestrained specimen was initially crept to $\epsilon \approx 0.2$ in the exponential creep region at $473 \mathrm{~K}$ under a normalized stress of $7.5 \times 10^{-4}$. The prestrained microstructure was similar to Fig. 16 (d) (Raj et al., 1989) (with kind permission from Elsevier Science Ltd.).

TABLE III-COMPARISON OF THE AVERAGE SIZES OF CELLS AND SUBGRAINS FORMED IN NaCl SINGLE CRYSTALS AT $873 \mathrm{~K}$ UNDER A STRESS OF 1.0 MPa IN THE CLASS M REGIME IN PRESTRAINED AND AS-RECEIVED SPECIMENS. (Raj et al., 1989)

\begin{tabular}{|c|c|c|c|}
\hline $\begin{array}{l}\text { Material } \\
\text { condition }\end{array}$ & $\begin{array}{c}\text { Test } \\
\text { parameters }\end{array}$ & $\begin{array}{l}\mathrm{d}_{\mathrm{c}}, \\
\mu \mathrm{m}\end{array}$ & $\begin{array}{l}\mathrm{d}_{\mathrm{s}}, \\
\mu \mathrm{m}\end{array}$ \\
\hline As-received & $\begin{array}{c}\mathrm{T}=873 \mathrm{~K} \\
\sigma=1.0 \mathrm{MPa} \\
\varepsilon=0.40\end{array}$ & $19.5 \pm 1.8$ & $193.5 \pm 30.5$ \\
\hline $\begin{array}{c}\text { Prior creep at } \\
\mathrm{T}=473 \mathrm{~K} \text { and } \\
\sigma=10.0 \mathrm{MPa} \\
\varepsilon=0.2\end{array}$ & $\begin{array}{c}\mathrm{T}=873 \mathrm{~K} \\
\sigma=1.0 \mathrm{MPa} \\
\varepsilon=0.43\end{array}$ & $19.1 \pm 2.1$ & $126.3 \pm 35.3$ \\
\hline
\end{tabular}

The sigmoidal primary creep observed in the prestrained material (Fig. 36) is analogous to similar behavior reported for $\mathrm{Cu}-16$ at.\% Al (Fig. 32) (Hasegawa et al., 1972). Therefore, a similar rationale can be adopted to explain the nature of the creep curve for the prestrained specimen. The high dislocation density representative of that in the exponential creep region (Fig. 16(d)) suggests that few mobile dislocations are available initially for measurable creep to occur. However, as the dislocation substructure begins to recover, the density of mobile dislocations increases, which leads to the initial rise in the creep rate observed in Fig. 36. The subsequent decrease in the creep rate beyond the peak value can be attributed to an increase in the long range internal stress in the specimen as cells and subgrains evolve during deformation.

In the second series of experiments, a specimen was first crept in the class $\mathrm{M}$ creep regime in Fig. 10. The microstructure after this deformation consisted of large equiaxed primary subgrains and cells (Fig. 38). Next, the specimen was retested at $473 \mathrm{~K}$ under a stress of $10.0 \mathrm{MPa}\left(\sigma / \mathrm{G}=7.5 \times 10^{-4}\right)$, where the stress and temperature conditions correspond to the exponential creep regime in Fig. 10. The creep rates for the prestrained specimen were significantly lower than those for an as-received specimen deformed under similar stress and temperature conditions (Fig. 39). The prestrained specimen was removed for microstructural examination at points X and $\mathrm{Y}$ marked on the lower curve in Fig. 39. 

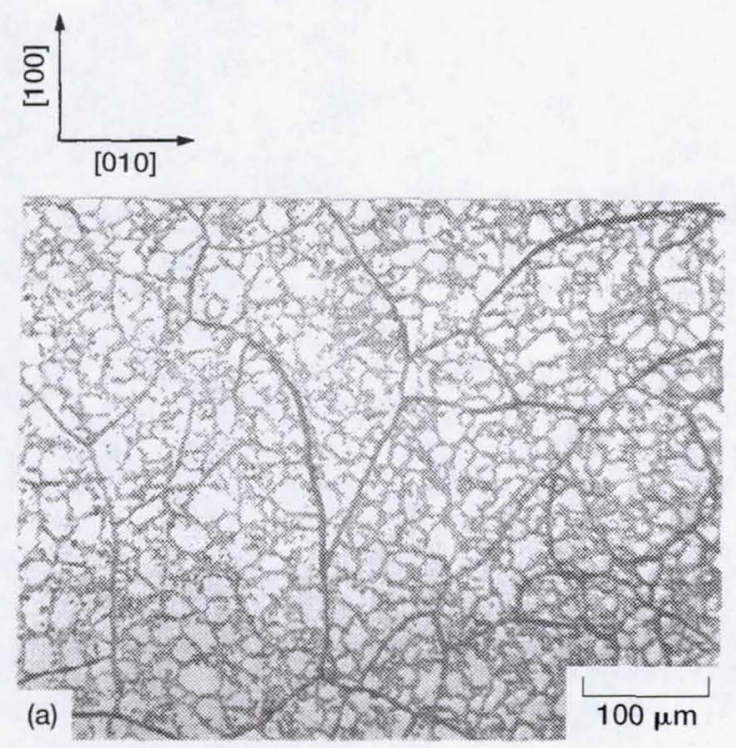

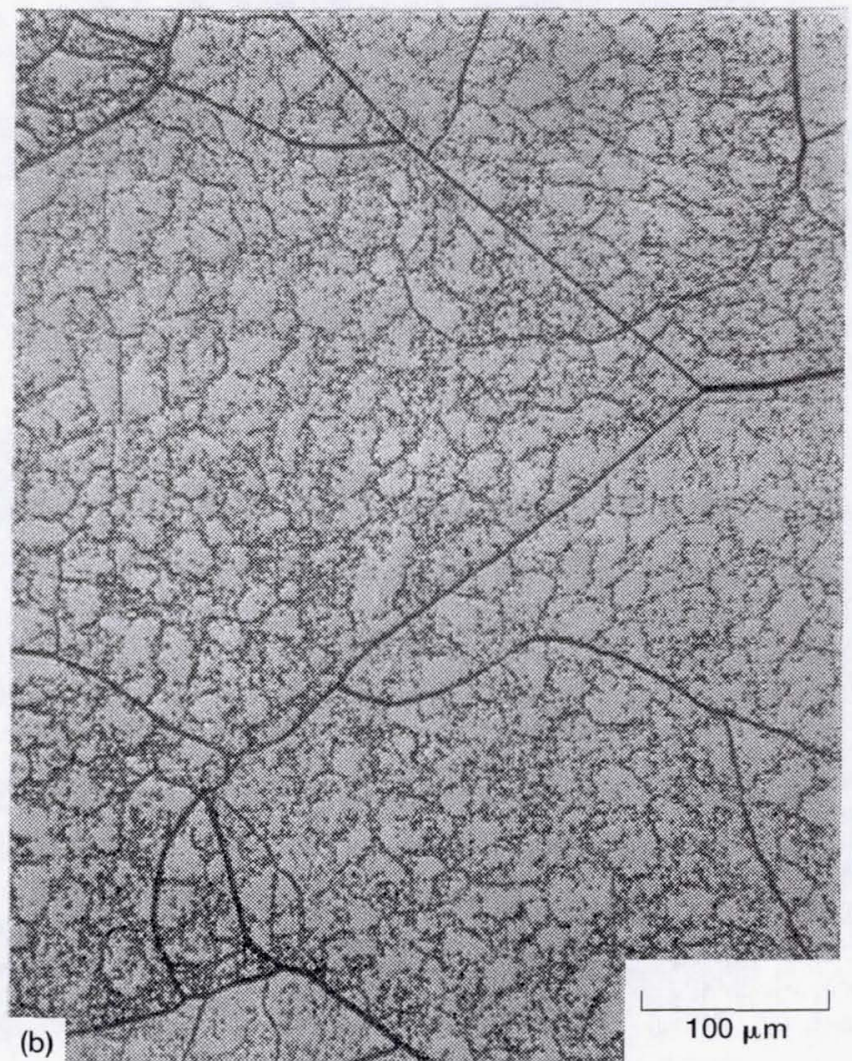

(b)

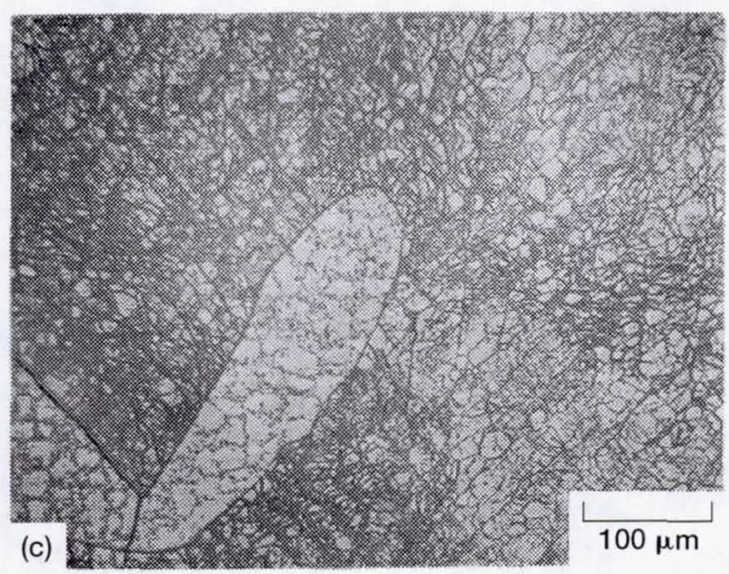

Figure 37.-(a) Microstructure of a sodium chloride single crystal specimen prestrained in the exponential creep regime and subsequently deformed to $\epsilon \approx 0.43$ at $843 \mathrm{~K}$ under a normalized stress of $1.1 \times 10^{-4}$ corresponding to the class $\mathrm{M}$ creep regime. Equiaxed primary subgrains, secondary subboundaries and cells have formed from the initial microstructure shown in Fig. 16 (d). (b) Microstructure of an as-received specimen deformed to $\epsilon \approx 0.40$ at $843 \mathrm{~K}$ under a normalized stress of $1.1 \times 10^{-4}$. (c) Effect of static annealing at $843 \mathrm{~K}$ on the prestrained microstructure shown in Fig. 16 (d) (i. e. $\sigma=0$ ). Comparison of Figs. 37 (a) and (c) reveals that the applied stress is the major driving force for substructure formation during creep (Raj et al., 1989) (with kind permission from Elsevier Science Ltd.). 


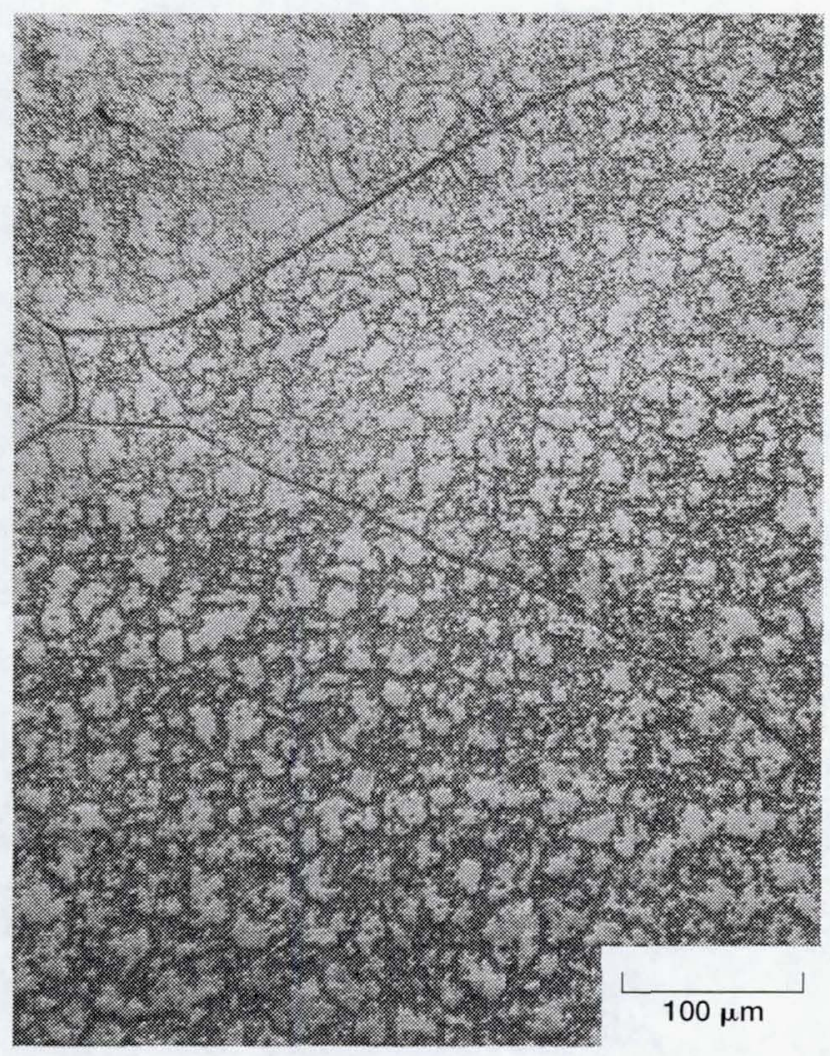

Figure 38.-Microstructure of a sodium chloride single crystal specimen prestrained to $\epsilon \approx 0.20$ at $923 \mathrm{~K}$ under a normalized stress of $7.5 \times 10^{-5}$ in the class $M$ regime (Raj et al., 1989) (with kind permission from Elsevier Science Ltd.).

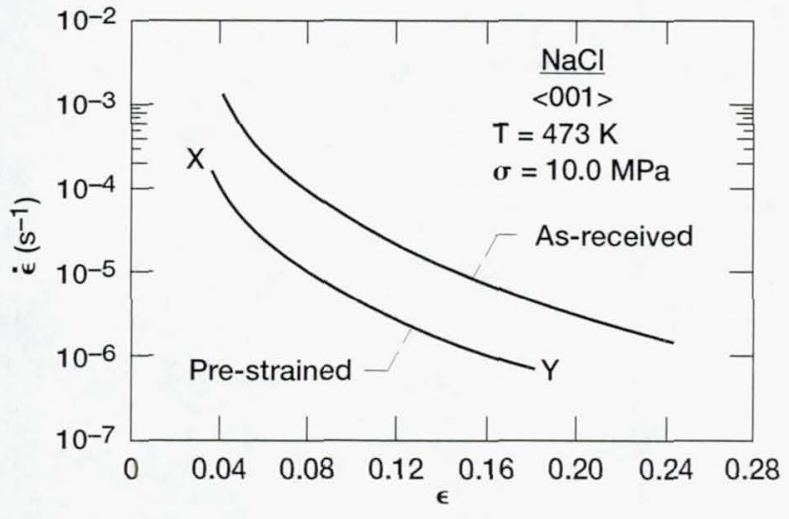

Figure 39.-Comparison of the creep curves for asreceived and prestrained sodium chloride specimens deformed at $473 \mathrm{~K}$ under a normalized stress of $7.5 \times 10^{-4}$ corresponding to the exponential creep regime. The prestrained specimen was initially crept to $\epsilon \approx 0.2$ at $923 \mathrm{~K}$ under a normalized stress of $7.5 \times 10^{-5}$ corresponding to the class $\mathrm{M}$ regime. Microstructural observations were conducted on the prestrained specimen after deformation to points marked ' $X$ ' and ' $Y$ ' (Raj et al., 1989) (with kind permission from Elsevier Science Ltd.).

Soon after loading (i.e., $\mathrm{X}$ in Fig. 39), the surface microstructure of the prestrained specimen perpendicular to the stress axis showed evidence of slip bands traversing the original subboundaries (Fig. 40(a)). On repolishing and re-etching the surface, it was found that the original subboundaries were still intact and there was no evidence of any catastrophic rupture of these boundaries anywhere on the surface of observation (Fig. 40(b)). However, localized migration of the subboundaries had occurred in some regions (e.g., A in Fig. 40(b)). The observation that the subboundaries are mechanically stable directly contradicts theoretical predictions that exponential creep is due to the mechanical instability of subboundaries (Pharr, 1981). Instead, the cellular microstructure, initially present within the primary subgrains (Fig. 38), was more or less replaced by an uniform distribution of etch pits presumably due to the rupture of the original cell boundaries; however, the generation of fresh dislocations from newly activated sources at the higher stress can also account for some of this increase in the dislocation density.

Continued deformation to $\varepsilon \approx 0.18$ (i.e., Y in Fig. 39 ) resulted in the formation of new subboundaries and in the localized migration of the original subgrain boundaries (e.g., arrows in Fig. 41(a)). It was observed that the migration of the old subboundaries was partly responsible for nucleating new subgrains. For this to happen, the subboundary segment must bow beyond a critical radius, $\mathrm{d}_{\mathrm{sb}} / 2 \approx \beta_{1} \Gamma_{\mathrm{sb}} / \tau$, where $\mathrm{d}_{\mathrm{sb}}$ is the subgrain diameter of the new subgrains, $\Gamma_{\mathrm{sb}}$ is the surface energy of a subboundary and $\beta_{1}$ is a constant. In many areas of the specimen, the random dislocation microstructure shown in Fig. 40(b) was found to have been completely replaced by well developed subgrains (Fig. 41(b)). It should be noted that this microstructure is vastly different than the one observed in an asreceived specimen deformed to $\varepsilon \approx 0.2$ under similar stress and temperature conditions (Fig. 16(d)). However, a mixture of elongated and equiaxed subgrains were observed in an as-received specimen after deformation to $\varepsilon \approx 0.5$ (Fig. 26(c)). Therefore, the creep curve for the prestrained specimen in Fig. 39 can be viewed as a lateral shift of the $\dot{\varepsilon}-\varepsilon$ plot to lower values of strain relative to the unstrained material. In effect, the ready evolution of a subgrain microstructure in the predeformed specimen also results in the development of a larger internal back stress in comparison to the as-received material. 

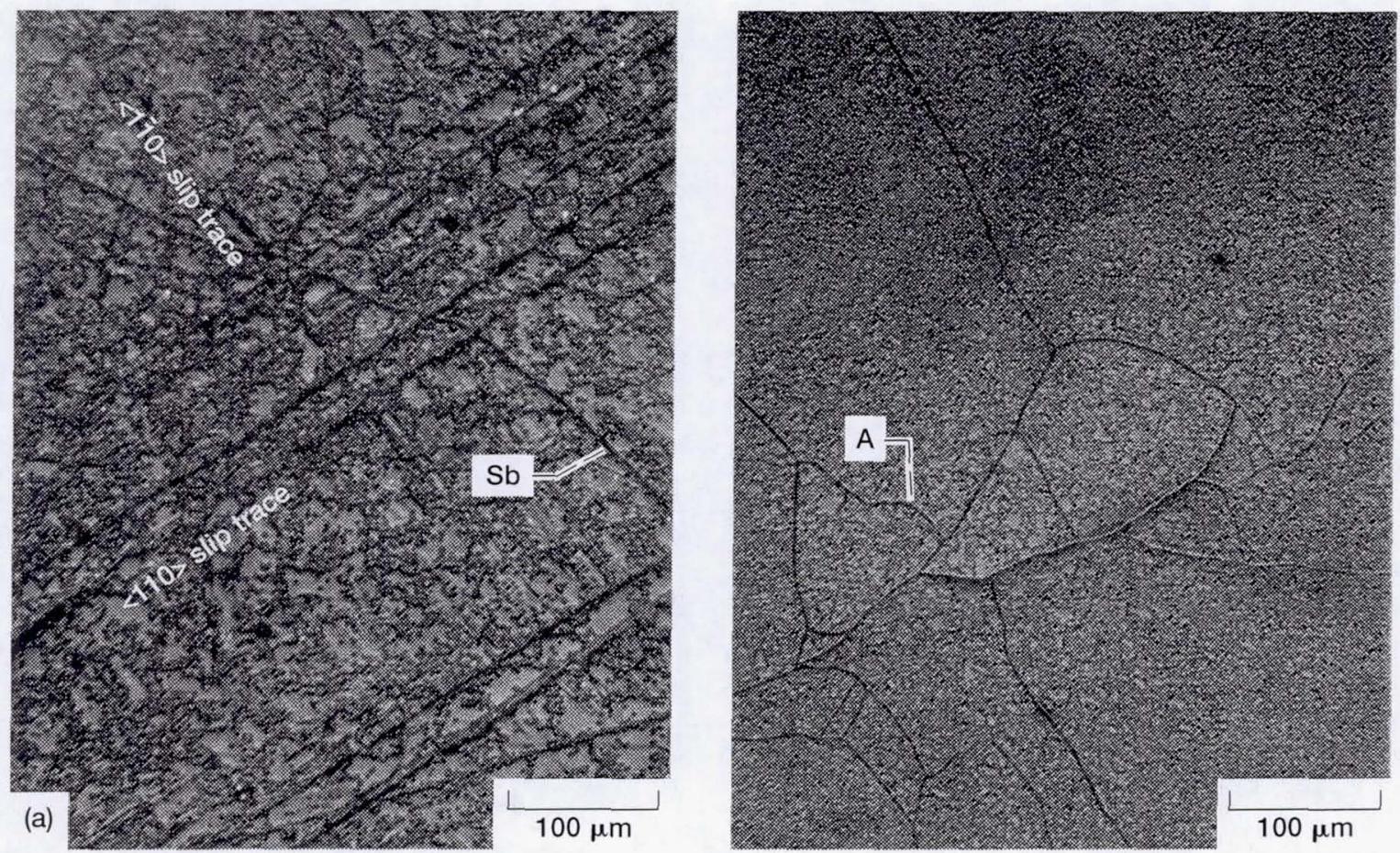

Figure 40.-Microstructures at the point marked ' $X$ ' in Fig. 39; $\epsilon \approx 0.034, T=473 \mathrm{~K}, \sigma / \mathrm{G}=7.5 \times 10^{-4}$. (a) Unpolished and etched surface showing slip lines crossing a subboundary $(\mathrm{Sb})$ originally formed after prior creep in the class $\mathrm{M}$ regime. (b) Polished and etched surface demonstrating that the original subboundaries after prestraining are mechanically intact. Local subboundary migration has occurred at some regions (e. g. at A) and the dislocation density in the subgrain interior has increased considerably compared to the prestrained microstructure shown in Fig. 38 (Raj et al., 1989) (with kind permission from Elsevier Science Ltd.).

The above observations on the effect of prior deformation lead to some significant conclusions. First, microstructures formed in the exponential creep regime are inherently unstable and they will continue to transform towards a steady-state substructure. The first series of experiments described in this section supports this conclusion, which also provides an experimental basis for Fig. 28. Second, subboundaries exhibit considerable mechanical stability and do not breakdown catastrophically into individual dislocations under high stress. Instead, a stress increase is accommodated by subboundary migration, the probable break-up of cell boundaries and the activation of new dislocation sources. Third, this irreversible nature of the subgrain microstructure also leads to a path dependent flow behavior. In other words, the transition from the exponential region to the class $\mathrm{M}$ creep is irreversible and path dependent.

\subsection{NIX-GIBELING ONE-DIMENSIONAL TWO-PHASE CREEP MODEL}

As discussed in $\S 3$ and 4 , the deformation behavior of materials is influenced by the microstructure and the microstructure in turn develops in response to external variables such as stress, temperature and the initial microstructure. Thus, there is a tendency towards steady-state flow behavior as the deformation substructure tends towards an equilibrium configuration. It was also demonstrated that the deformation substructure is heterogeneous in nature consisting of 'hard' and 'soft' regions with different deformation responses to the applied stress. The 'hard' regions consist of elongated subgrains with a high dislocation density within them (e.g., A in Fig. 16(b)), cell and subgrain boundaries, and highly clustered regions with large dislocation densities. In contrast, the "soft" regions consist of equiaxed subgrains with a low dislocation density within them (e.g., B in Fig. 16(b)), cell and subgrain interiors, and regions of the crystal with low dislocation densities.

Although it has long been recognized that creep is influenced by the microstructure, initial attempts to model creep deformation have failed to recognize the heterogeneous nature of the creep substructure. Thus, these 

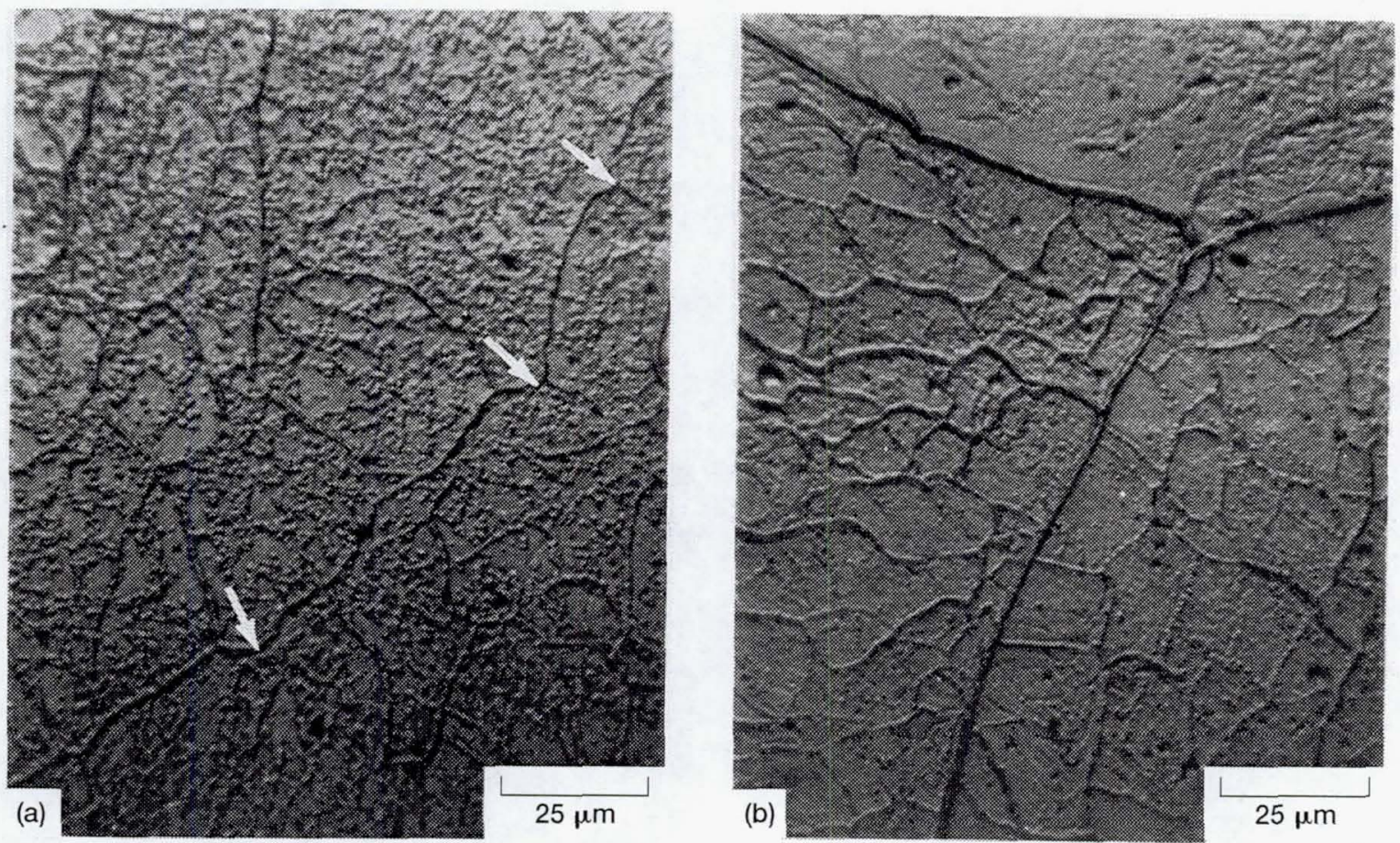

Figure 41.-Microstructures at the point marked ' $Y$ ' in Fig. 39; $\epsilon \approx 0.18, T=473 \mathrm{~K}, \boldsymbol{\sigma} / \mathrm{G}=7.5 \times 10^{-4}$. (a) The original subboundaries show evidence of localized migration as indicated by the arrows and recovery within the subgrain interior. (b) New subgrains have formed within the original subgrains in part due to subboundary migration (Raj et al., 1989) (with kind permission from Elsevier Science Ltd.).

models have largely ignored contributions from processes other than dislocation climb so that the ensuing rate equation always resulted in $n=3$, if no special assumptions are made, no matter which microstructural feature is considered to be important in the model (Weertman, 1975). However, as mentioned earlier, experimental observations generally result in a value of $\mathrm{n}>3$ (Bird et al., 1969).

The first serious attempt to develop a creep model based on the heterogenous nature of the dislocation substructure was proposed by Nix and Ilschner (1980). Simultaneously, Mughrabi (1980) and Mughrabi and Essmann (1980) also considered the heterogeneity in the microstructure in understanding low temperature and cyclic deformation. These approaches largely recognize the different deformation characteristics of the 'hard' and 'soft' regions. Subsequently, several other models have been developed for uniaxial and cyclic deformation using a 'hard' and 'soft' microstructural-based deformation concepts (Mughrabi, 1981; Mughrabi, 1983; Vogler and Blum, 1990; Argon and Haasen, 1993; Hofmann and Blum, 1993; Zhu and Blum, 1993). Typically, these models consider two microstructural parameters involving the dislocation density and the cell or subgrain size.

Nix and Ilschner (1980) attempted to model class M and exponential creep behavior by assuming that the creep rate is given by

$$
\dot{\varepsilon}=\dot{\varepsilon}_{\mathrm{g}}+\dot{\varepsilon}_{\mathrm{cl}}
$$

where $\dot{\varepsilon}_{\mathrm{g}}$ is the creep rate for thermally activated glide given by equation (11) and $\dot{\varepsilon}_{\mathrm{cl}}$ is the dislocation climb-controlled creep rate given by equation (3). It was assumed that $\dot{\varepsilon}_{\mathrm{g}}$ is the creep rate in the 'soft' regions of the subgrain interior while $\dot{\varepsilon}_{\mathrm{cl}}$ is the creep rate in the 'hard' regions of the subboundaries. Although equation (24) suggests that the class $\mathrm{M}$ to exponential creep transition will occur naturally as a consequence of the dominance of nondiffusional creep mechanisms, its formulation is fundamentally flawed since it treats the deformation of the 'hard' and 'soft' regions as independent of each other.

Subsequently, while preserving the concept of 'hard' and 'soft' regions, Nix et al., (1985 (a) and (b)) proposed a two-phase composite model for creep. This approach treats the microstructure as a composite of 'hard' and 'soft' regions, where the deformation occurring in the cell or subgrain interior is coupled with that taking place in 
the cell or subgrain boundaries (Fig. 42). The model assumes that screw dislocations glide, multiply, cross-slip and annihilate in the "soft" regions, while simultaneously depositing two edge components at the cell or subgrain walls. Recovery is assumed to occur at elevated temperatures by the climb and annihilation of the dislocations in the cell or subgrain walls. The original model was strictly valid for high stacking fault energy materials. However, a modified derivation is presented here which should be applicable for low stacking fault energy materials as well.

\subsection{Modified Nix-Gibeling Two-Phase Composite Model}

The modified approach is shown in Fig. 43(a). The screw dislocations of unit Burgers vector, $\vec{b}$, are assumed to be separated into two partial dislocations of Burgers vectors, $\vec{b}_{1}$ and $\vec{b}_{2}$, respectively, in the cell or subgrain interior. These dislocations are assumed to glide in the extended state, while depositing edge components at the boundaries by forming constrictions at the points marked 'A' (Fig. 43(b)). Subsequently, these edge components are assumed to split into partial dislocations in the cell or subgrain walls. It follows from Fig. 43(a) that recovery within the cell or subgrain interiors can occur by the cross-slip of the screw components after the two partials form a constriction. In comparison, recovery in the cell or subgrain boundaries regions can occur by climb of the extended dislocations in the walls or to a limited extent by cross-slip of the screw components at A (Fig. 43(c)). Dislocation glide in the cell walls is assumed to occur along the $\mathrm{X}$ direction. In order to maintain compatibility and using the Voigt approximation,

$$
\dot{\gamma}=\dot{\gamma}^{\mathrm{ps}}+\dot{\tau}^{\mathrm{s}} / \mathrm{G}=\dot{\gamma}^{\mathrm{ph}}+\dot{\tau}^{\mathrm{h}} / \mathrm{G}
$$

where $\dot{\gamma}^{\mathrm{ps}}$ and $\dot{\gamma}^{\mathrm{ph}}$ are the plastic strain rates in the 'soft' (s) and the "hard' (h) regions, respectively, and $\dot{\tau}^{\mathrm{s}} / \mathrm{G}$ and $i^{\mathrm{h}} / \mathrm{G}$ are the elastic shear strain rates in the 'soft' and 'hard' phases, respectively. A second condition that must be satisfied in order to maintain mechanical equilibrium leads to (Mughrabi, 1983)

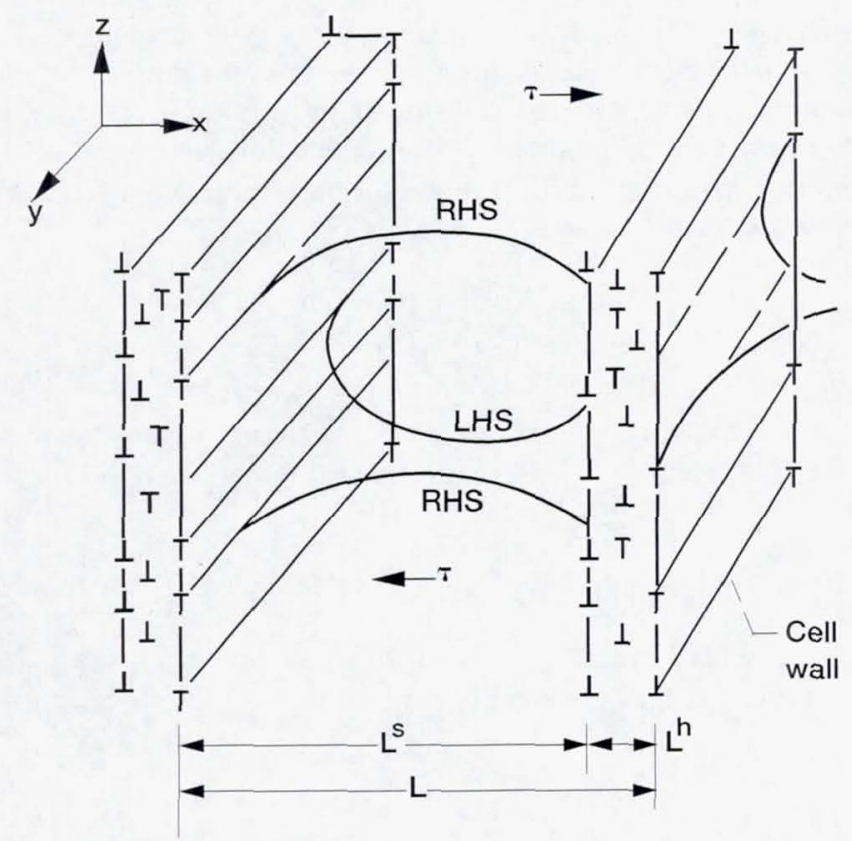

Figure 42.- Schematic illustration of a one-dimensional cell consisting of infinitely long cell walls. Screw dislocations glide in the cell interior and deposit edge dislocations in the cell walls (Nix and Gibeling, 1985; Nix et al., 1985) (courtesy Metallurgical and Materials Transactions). 


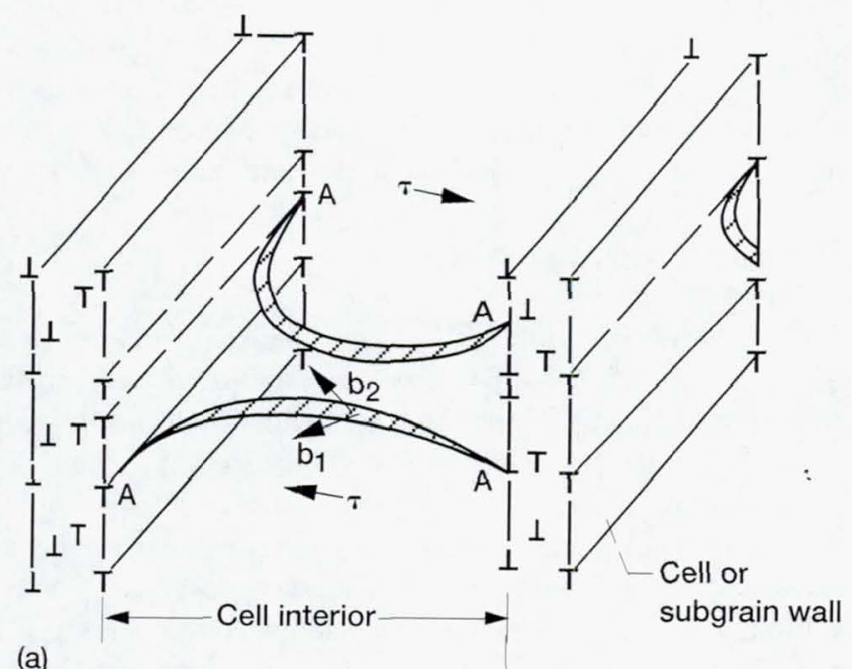

(a)

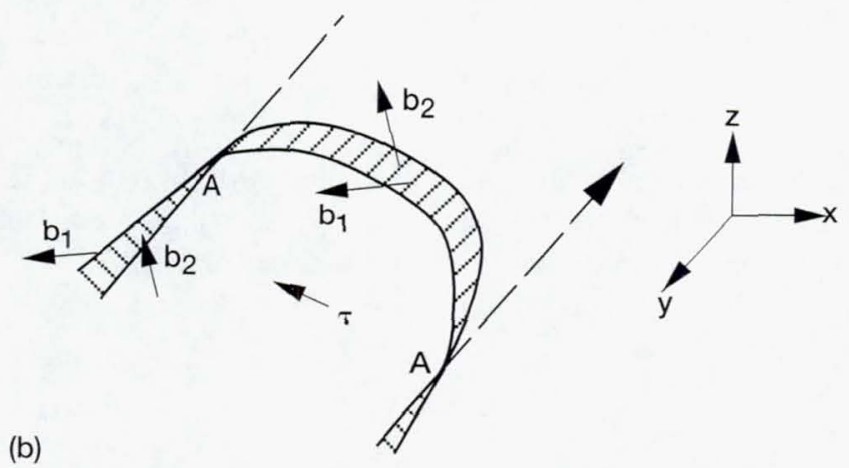

Figure 43.-(a) Schematic illustration of the one-dimensional cell model shown in Fig. 42 for the case when the dislocations are split into partial dislocations. (b) Detailed view of the split dislocations of Burgers vectors $\overrightarrow{b_{1}}$ and $\overrightarrow{b_{2}}$ showing their constriction at $A$ and further separation in the cell walls.

$$
\tau=\mathrm{f}^{\mathrm{h}} \tau^{\mathrm{h}}+\mathrm{f}^{\mathrm{s}} \tau^{\mathrm{s}}
$$

where $\tau^{s}$ and $\tau^{h}$ are the effective shear stresses acting in the 'soft' and 'hard' regions, respectively, and $f^{s}$ and $f^{h}$ are the volume fractions of the 'soft' and the 'hard' regions, respectively, so that

$$
\mathrm{f}^{\mathrm{h}}+\mathrm{f}^{\mathrm{s}}=1
$$

Noting that $\tau^{\mathrm{h}}=\tau+\tau_{\mathrm{f}}$ and $\tau^{\mathrm{s}}=\tau-\tau_{\mathrm{b}}$, where $\tau_{\mathrm{f}}$ and $\tau_{\mathrm{b}}$ are the forward and the back shear stresses acting on the 'hard' and 'soft' regions, respectively, equations (26) and (27) lead to

$$
\tau_{\mathrm{f}}=\left(\mathrm{f}^{\mathrm{s}} / \mathrm{f}^{\mathrm{h}}\right) \tau_{\mathrm{b}}
$$

As shown in Fig. 35, the forward stress is several times greater than the back stress implying that $f^{s}>f^{\text {h }}$.

Assuming that dislocation glide in the 'soft' and 'hard' regions occurs by the intersection of rectangular obstacles, the strain rates in the two regions can be formulated in terms of equation (11) as (Nix and Gibeling, 1985(a)) 


$$
\dot{\gamma}^{\mathrm{p} \phi}=4 \times 10^{12}\left(\hat{\tau}^{\phi} / \mathrm{G}\right)^{2} \exp \left[-(\Delta \mathrm{F} / \mathrm{kT})\left(1-\tau^{\phi} / \hat{\tau}^{\phi}\right)\right]
$$

where $\phi=$ ' h' or 's' and $\hat{\tau}^{\phi}$ is the shear strength of the obstacle in each region related to the appropriate dislocation density, $\rho^{\phi}$, by (Taylor, 1934)

$$
\hat{\tau}^{\phi}=\alpha^{\phi} / \mathrm{Gb}\left(\rho^{\phi}\right)^{0.5}
$$

As deformation progresses, the strength of the obstacles constantly evolves in the two regions and it is necessary to establish the structure evolution laws. Screw dislocations glide in the cell interior until they are stopped by the elastic fields of other dislocations. Strain hardening occurs as these dislocations are statistically stored in the cell interior. The plastic strain increment associated with this hardening event is given by

$$
\mathrm{d} \gamma^{\mathrm{ps}}=\mathrm{b} \Lambda^{2} \mathrm{~d} \rho^{\mathrm{s}}
$$

where $\Lambda^{\mathrm{s}}$, the average distance travelled by a dislocation in the cell interior before it is stopped, has a value of about $100 /\left(\rho^{\mathrm{s}}\right)^{0.5}$. The rate of increase in the dislocation density is then

$$
\left(\dot{\rho}^{\mathrm{s}}\right)^{+}=\left[\left(\sqrt{\rho^{\mathrm{s}}}\right) / 100 \mathrm{~b}\right] \dot{\gamma}^{\mathrm{ps}}
$$

The rate of decrease in the dislocation density, $\left(\dot{\rho}^{\mathrm{s}}\right)^{-}$, in the cell interior due to cross-slip and annihilation of two screw dislocations is given by (Nix et al., 1985(b))

$$
\left(\dot{\rho}^{s}\right)^{-}=-20\left[\left(\sqrt{\rho^{s}}\right) / L^{*}\right] v_{D} \exp \left(-\Delta G_{C S} / k T\right)
$$

where $v_{D}$ is the Debye frequency and $L^{*}$ is the activated length for nucleating a single cross-slip event. Nix et al., (1985(a) and (b)) assumed that $\mathrm{L}^{*}=1000 \mathrm{~b}$ but this is unrealistically large. Noting that $\mathrm{A}^{*} \approx 300 \mathrm{~b}^{2}$ for cross-slip (Conrad, 1964; Evans and Rawlings, 1969; Bonneville and Escaig, 1979), and assuming a stacking fault width of 5 to $10 \mathrm{~b}$, it is more likely that $\mathrm{L}^{*} \approx 30$ to $60 \mathrm{~b}$. Using equations (32) and (33), it can be shown that the rate of change of the dislocation density in the 'soft' region, $\dot{\rho}$ ', is given by

$$
\dot{\rho}^{\mathrm{s}}=\left(\sqrt{\rho^{\mathrm{s}}} / 100 \mathrm{~b}\right) \dot{\gamma}^{\mathrm{ps}}-20\left(\sqrt{\rho^{\mathrm{s}}} / \mathrm{L}^{*}\right) v_{\mathrm{D}} \exp \left(-\Delta \mathrm{G}_{\mathrm{CS}} / \mathrm{kT}\right)
$$

The rate of change in the obstacle strength in the cell interior is given by (Nix et al., 1985(a) and (b))

$$
\mathrm{d} \hat{\tau}^{\mathrm{s}} / \mathrm{d} \gamma^{\mathrm{ps}}=\mathrm{G} / 200-10\left(\mathrm{~b} / \mathrm{L}^{*}\right) \nu_{\mathrm{D}}\left(\mathrm{G} / \dot{\gamma}^{\mathrm{ps}}\right) \exp \left(-\Delta \mathrm{G}_{\mathrm{CS}} / \mathrm{kT}\right)
$$

The first term in equation (35) represents the linear work hardening rate while the second term accounts for recovery within the cell or subgrain interior due to the annihilation of screw dislocations by cross slip. The inclusion of the recovery term accounts for the finite probability that some of the screw dislocations can form a constriction according to the Friedel-Escaig mechanism (Friedel, 1959, 1964 1977; Escaig, 1968(a) and (b)). The activation free energy for cross-slip is given by equation (10). It is noted that $Q_{01}$ in equation (10) increases as the stacking fault energy decreases through its inverse dependence on the adjustable parameter $\alpha$ ', so that the probability of forming constrictions and the rate of recovery by cross slip also decrease with $\Gamma / \mathrm{Gb}$. As noted earlier, it is expected that $\mathrm{Q}_{01} \approx 185 \mathrm{~kJ} \mathrm{~mol}^{-1}$ and $\alpha^{\prime} \approx 500$ to 950 for NaCl single crystals based on the experimental data shown in Fig. 7 . Alternatively, the activation energy can be expressed in terms of equation (8). 
The glide of a screw dislocation in the cell interior deposits two edge components at the cell walls. Strain hardening in the cell walls occurs as these edge dislocations knit into the walls with a corresponding increase in the stress in the cell walls. The strain increment due to this process is similar to that given by equation (31) except that the distance, $\Lambda^{\mathrm{h}}$, traversed by the dislocation in the cell walls is expected to be equal to the mesh spacing, $h$, in the walls. The dislocation spacing in the boundaries is given by $\Lambda^{h} \approx h=\left(K_{1} G b / \hat{\tau}^{h}\right)$ and $K_{1}$ is a constant equal to about 5. The rate of increase in the dislocation density in the cell walls is then

$$
\left(\dot{\rho}^{\mathrm{h}}\right)^{+}=\left[\left(\sqrt{\rho^{\mathrm{h}}}\right) / \mathrm{K}_{1} \mathrm{~b}\right] \dot{\gamma}^{\mathrm{ph}}
$$

where $\rho^{h}$ is the dislocation density in the cell walls.

Assuming that the recovery in the walls occurs only by the climb and annihilation of the extended dislocations, the rate of decrease of redundant dislocations in the cell walls is given by (Prinz, et al., 1982)

$$
\left(\dot{\rho}^{\mathrm{h}}\right)^{-}=-2 \rho^{\mathrm{h} / \mathrm{t}} \mathrm{C}
$$

where $\mathrm{t}_{\mathrm{C}}$ is the characteristic time required for a pair of edge dislocations to climb a distance $\mathrm{h} / 2$ in the cell or subgrain boundary before annihilating each other. The characteristic time is given by $t_{C}=h / 2 v_{C}$, where $v_{C}$ is the climb velocity of the extended dislocations. Following Argon and Moffatt (1981)

$$
\mathrm{v}_{\mathrm{C}}=\beta_{2} \mathrm{C}_{\mathrm{j}} \mathrm{b}(\Gamma / \mathrm{Gb})^{2}\left(\mathrm{D}_{1} \mathrm{G} \Omega / \mathrm{kTb}\right)\left(\hat{\tau}^{\mathrm{h}} / \mathrm{G}\right)
$$

where $C_{i}$ is the density of extended jogs per unit length along the dislocation line, and $\beta_{2}$ is a constant equal to about 1000. Although recent studies suggest that $C_{j}$ is dependent on $\Gamma / G b$ through a complex power-law relation (Kong and $\mathrm{Li}, 1993$ ), the magnitudes of several parameters used in the derivation of $\mathrm{C}_{\mathrm{j}}$ are unknown. The magnitude of $\mathrm{C}_{\mathrm{j}}$ is assumed to be independent of $\Gamma / \mathrm{Gb}$ in the present paper for simplicity. Assuming $\Omega \approx 0.7 \mathrm{~b}^{3}$ and using equation (30) with $\alpha^{\phi}=\alpha^{h}$, the rate of decrease in the dislocation density in the cell walls is

$$
\left(\dot{\rho}^{h}\right)^{-} \approx-\left(1.4 \beta_{2} C_{j} b / K_{1}\right)\left(\alpha^{h}\right)^{2}(\Gamma / G b)^{2}\left(D_{1} G^{3} / k T\right)\left(\rho^{h}\right)^{2}
$$

The total rate of change in the dislocation density in the cell walls is obtained by combining equations (37) and (39)

$$
\left(\dot{\rho}^{\mathrm{h}}\right)=\left[\left(\sqrt{\rho^{\mathrm{h}}}\right) / \mathrm{K}_{1} \mathrm{~b}\right] \dot{\gamma}^{\mathrm{ph}}-\left(1.4 \beta_{2} \mathrm{C}_{\mathrm{j}} \mathrm{b} / \mathrm{K}_{1}\right)\left(\alpha^{\mathrm{h}}\right)^{2}(\Gamma / \mathrm{Gb})^{2}\left(\mathrm{D}_{1} \mathrm{~Gb}^{3} / \mathrm{kT}\right)\left(\rho^{\mathrm{h}}\right)^{2}
$$

Assuming $\mathrm{K}_{1} \approx 5, \beta_{2} \approx 1000$ (Argon and Moffatt, 1981) and $\mathrm{C}_{\mathrm{j}} \mathrm{b} \approx 0.01$ (Prinz et al., 1982), equation (40) reduces to

$$
\left(\dot{\rho}^{\mathrm{h}}\right)=\left[\left(\sqrt{\rho^{\mathrm{h}}}\right) / 5 \mathrm{~b}\right] \dot{\gamma}^{\mathrm{ph}}-(2.8)\left(\alpha^{\mathrm{h}}\right)^{2}(\Gamma / \mathrm{Gb})^{2}\left(\mathrm{D}_{1} \mathrm{~Gb}^{3} / \mathrm{kT}\right)\left(\rho^{\mathrm{h}}\right)^{2}
$$

The rate of change of the obstacle strength in the 'hard' regions is given by

$$
\mathrm{d} \hat{\tau}^{\mathrm{h}} / \mathrm{d} \gamma^{\mathrm{ph}}=\left(\mathrm{d} \hat{\tau}^{\mathrm{h}} / \mathrm{d} \gamma^{\mathrm{ph}}\right)^{+}+\left(\mathrm{d} \hat{\tau}^{\mathrm{h}} / \mathrm{d} \gamma^{\mathrm{ph}}\right)^{-}
$$

where $\left(\mathrm{d} \hat{\tau}^{\mathrm{h}} / \mathrm{d} \gamma^{\mathrm{ph}}\right)^{+}$is the increase in the obstacle strength in the walls due to the deposition of the edge components and $\left(\mathrm{d} \hat{\tau}^{\mathrm{s}} / \mathrm{d} \gamma^{\mathrm{ph}}\right)^{-}$is the rate of decrease in the obstacle strength in the walls due to recovery. Noting that (Haasen, et al., 1986) 


$$
\left(\mathrm{d} \hat{\tau}^{\mathrm{h}} / \mathrm{d} \gamma^{\mathrm{ph}}\right)^{+}=\left(\mathrm{d} \hat{\tau}^{\mathrm{h}} / \mathrm{d} \rho^{\mathrm{ph}}\right)(\dot{\rho})^{+}\left(1 / \dot{\gamma}^{\mathrm{ph}}\right)
$$

The second term in equation (42) can be expressed as

$$
\left(\mathrm{d} \hat{\tau}^{\mathrm{h}} / \mathrm{d} \dot{\gamma}^{\mathrm{ph}}\right)^{-}=\left(\mathrm{d} \hat{\tau}^{\mathrm{h}} / \mathrm{dt}\right)^{-}\left(1 / \dot{\gamma}^{\mathrm{ph}}\right)
$$

where

$$
\left(\mathrm{d} \hat{\tau}^{\mathrm{h}} / \mathrm{dt}\right)^{-}=\left[\left(\alpha^{\mathrm{h}} \mathrm{Gb}\right)^{2} / 2 \hat{\tau}^{\mathrm{h}}\right]\left(\dot{\rho}^{\mathrm{h}}\right)^{-}
$$

Combining equations (36), (39) and (42) to (45), the rate of change in the obstacle strength in the boundaries is

$$
\left(\mathrm{d} \hat{\tau}^{\mathrm{h}} / \mathrm{d} \gamma^{\mathrm{ph}}\right)=\left(\alpha^{\mathrm{h}}\right)(\mathrm{G} / 10)-1.4(\Gamma / \mathrm{Gb})^{2}\left(\mathrm{D}_{1} \mathrm{~Gb} / \mathrm{kT}\right)\left(\hat{\tau}^{\mathrm{h}} / \mathrm{G}\right)^{3}\left(\mathrm{G} / \dot{\gamma}^{\mathrm{ph}}\right)
$$

The second term in equation (46) exhibits the natural third power-law dependence on stress associated with many creep theories (Weertman, 1975). The present treatment differs from that derived by Nix et al., (1985(a) and (b)) in two important ways. First, the climb velocity is assumed to be dependent on the stacking fault energy so that $\Gamma / G b$ enters the model in a natural manner. Second, Nix et al., (1985(a) and (b)) assumed that dislocations in the cell wall glide a constant distance equal to $\mathrm{L}^{\mathrm{h}} \approx 100 \mathrm{~nm}$. This is an unrealistic assumption since it implies that the edge components do not interact with the dislocations forming the cell walls. Thus, this assumption has been discarded in the present approach. Instead, the glide distance is assumed to be related to the mesh spacing in the cell walls in order to allow the glide distance to change constantly during deformation as the microstructure evolves. It is important to note that the recovery processes occurring in the cell interior as well at the boundaries are affected by the magnitude of the stacking fault energy through its influence on cross slip and climb.

\subsection{Limitations of the One-Dimensional Composite Model}

Despite its usefulness in describing creep, the one-dimensional model is limited in a number of ways some of which were discussed by Nix et al., (1985(a) and (b)). First, the model unrealistically assumes that cell and subgrain boundaries extend infinitely in one direction, and therefore fails to account for the three-dimensional nature of the substructure. Second, the model is strictly applicable for the special dislocation boundaries and processes illustrated in Figs. 42 and 43. Thus, recovery events due to dislocation climb of edge components are ignored in the "soft" regions and those due to cross-slip of screw dislocations occurring in other types of boundaries are not considered. Since both edge and screw dislocation components are expected to be present in the 'hard' and 'soft' regions, the model artificially isolates cross-slip events to the cell or subgrain interior and climb to the cell or subgrain walls. Third, the model implicitly assumes that cells or subgrains are present from the beginning of a creep test and it does not consider the time dependent variation of $\mathrm{f}^{\mathrm{h}}$ and $\mathrm{f}^{\mathrm{s}}$ or the manner in which dislocations pattern themselves into dislocation clusters (Kubin, 1993). Fourth, in view of the discussion in $\$ 3$, the model assumes an idealized and over simplified picture of the creep substructure. Fifth, the model is strictly valid for single phase, coarse-grained materials and single crystals exhibiting class $\mathrm{M}$ behavior since all grain boundary effects are ignored. Sixth, the number of slip systems activated in the material are not explicitly considered although the model implicitly assumes that multiple slip results in high rates of dislocation storage. Despite these limitations, this simple model can explain the development of internal stresses within a creeping solid as a natural consequence of the development of a heterogeneous microstructure. As will be demonstrated in $\S 6.1$, the Nix-Gibeling model is the limiting case of the more realistic three-dimensional DSV analysis (Freed, et al., 1992). 


\subsection{DEVELOPMENT OF A MULTIPHASE THREE-DIMENSIONAL CREEP MODEL}

As discussed in $\S 5.2$, the one-dimensional, two-phase creep model has several limitations. Although several similar two-phase deformation models have been proposed (e.g., Mughrabi, 1981, 1983, 1987; and Vogler and Blum, 1991), they too are limited in a similar manner. Recently, Qian and Fan (1991) developed a three-dimensional viscoplastic model based on dislocation substructure, but they did not provide for substructure strain compatibility.

\subsection{Dislocation Substructure Viscoplasticity (DSV) Model}

The present three-dimensional model accounts for this strain compatibility by using the Budiansky and Wu (1962) self-consistent formalism with an Eshelby criterion (1957) for strain compatibility between the 'hard' and 'soft' regions. The model has been termed as the Dislocation Substructure Viscoplasticity (DSV) model (Freed et al., 1992). The result is a rate dependent viscoplastic theory that incorporates a self-consistent effect of dislocation substructure on material response. The internal state variables of this theory are the dislocation densities of the 'hard' and 'soft' regions, the average size of the dislocation substructure, and the relative volume fractions of the two regions. The inclusion of variable geometric or size effects as well as the potential applicability to the deformation of several microstructural features (e.g. cells and subgrains) are unique features of the present model.

Several assumptions have been made in the construction of the model. First, the self-consistent method of Budiansky and Wu (1962) adequately represents the 'hard' and 'soft' regions to a first approximation with both regions having identical elastic moduli. Second, the cells and subgrains are treated as equiaxed, isotropic spherical inclusions in the Eshelby (1957) analysis for strain compatibility. Third, deformation involves multiple slip systems so that the material is isotropic and the plastic strain rate of each region is coaxial with its deviatoric stress. Consequently, the constitutive equations of Prandtl (1924) and Reuss (1930) are taken to apply in each region (i.e., no back stress is considered in the macroscopic flow law). Fourth, the von Mises (1913) criterion is used to describe the topology of the nested set of flow surfaces. The von Mises equivalent stress and plastic strain rate are considered to correlate with the stress and plastic strain rate of a critically resolved slip system via Taylor's relation (1938). Fifth, the local plastic strain rates of the 'hard' and 'soft' regions are governed by dislocation mechanics, where the dislocation structure is assumed to evolve during deformation. Sixth, grain boundary effects are assumed to be negligible. For simplicity the model is developed for a two-phase cellular substructure, but the procedure can be extended to other substructures, including multiphase microstructures.

\subsection{Composite Three-Dimensional Model}

Through the volume averaging process, each global tensor field, say $\overline{\mathrm{X}}_{\mathrm{ij}}$, is related to its local fields $\mathrm{X}_{\mathrm{ij}}^{\phi}$ through the summation

$$
\bar{X}_{i j}=\sum_{\phi=1}^{n} f^{\phi} X_{i j}^{\phi} \quad \text { such that } \quad 1=\sum_{\phi=1}^{n} f^{\phi}
$$

where $\mathrm{f}^{\phi}$ is the relative volume fraction associated with field $\mathrm{X}_{\mathrm{ij}}^{\phi}$ of phase $\phi$ (i.e., each type of substructure, such as cells and subgrains). This is a rule of mixtures relation, where each local field of the continuum represents an integrated volume average of that field over its associated phase of a microcontinuum, which is designated by a unit cell. A bar '-' is placed over a variable to identify that it represents the volume average of its local variables over the entire unit cell. In principle, equation (47) should describe a microstructure of several cells and subgrains such as those illustrated schematically in Figs. 17 and 18(a). For simplicity, only a two phase composite structure consisting of cell walls and cell interiors is considered here for which equation (47) reduces to

$$
\bar{X}_{i j}=\left(1-f^{h}\right) X_{i j}^{s}+f^{h} X_{i j}^{h},
$$


where $\phi=\mathrm{h}$ or $\mathrm{s}$. It is cautioned that although the individual tensor fields are considered to add up according to the rule of mixtures, mappings from one field to another (e.g., constitutive equations) need not satisfy such a rule. The Prandtl-Reuss (Prandtl, 1924; Reuss, 1930) equations are used to relate the local stresses, $\sigma_{\mathrm{ij}}^{\phi}$, and strain rates, $\dot{\varepsilon}_{\mathrm{ij}}^{\phi}$ ' in phase, $\phi$, so that

$$
\begin{gathered}
\dot{\sigma}_{\mathrm{kk}}^{\phi}=3 \kappa\left(\dot{\varepsilon}_{\mathrm{kk}}^{\phi}-\alpha_{\mathrm{c}} \dot{\mathrm{T}} \delta_{\mathrm{kk}}\right)+\frac{\dot{\kappa}}{\kappa} \sigma_{\mathrm{kk}}^{\phi} \\
\dot{\mathrm{S}}_{\mathrm{ij}}^{\phi}=2 \mathrm{G}\left(\dot{\mathrm{E}}_{\mathrm{ij}}^{\phi}-\dot{\lambda}^{\phi} S_{\mathrm{ij}}^{\phi}\right)+\frac{\dot{\mathrm{G}}}{\mathrm{G}} S_{\mathrm{ij}}^{\phi}
\end{gathered}
$$

where

$$
S_{i j}^{\phi}=\sigma_{i j}^{\phi}-\frac{1}{3} \sigma_{\mathrm{kk}}^{\phi} \delta_{\mathrm{ij}}
$$

and

$$
E_{\mathrm{ij}}^{\phi}=\varepsilon_{\mathrm{ij}}^{\phi}-\frac{1}{3} \varepsilon_{\mathrm{kk}}^{\phi} \delta_{\mathrm{ij}}
$$

are the deviatoric stresses and strains, respectively, in phase $\phi, \kappa$ is the bulk modulus, $\alpha_{c}$ is the mean coefficient of thermal expansion, $\delta_{\mathrm{ij}}$ is the Kronecker delta, $\dot{\lambda}^{\phi}$ describes the kinetics of plastic flow, $\dot{\kappa}=(\partial \kappa / \partial \mathrm{T}) \dot{\mathrm{T}}$ and $\dot{\mathrm{G}}=$ $(\partial G / \partial T) \dot{T}$. These are the governing constitutive equations of a thermal-elastic-plastic isotropic Hookean material. In plasticity (i.e., rate independent condition) $\dot{\lambda}^{\phi}$ would be described through a consistency condition; whereas, in viscoplasticity (i.e., rate-dependent condition) $\dot{\lambda}^{\phi}$ is described through a kinetics equation (an equation of state). A significant but realistic assumption in the current treatment is that $T, \alpha_{c}, G$ and $\kappa$ are assumed to be identical in both the 'hard' and 'soft' regions, which substantially simplifies the ensuing theory.

Writing the flow equation for plastic straining as

$$
\dot{\varepsilon}_{\mathrm{ij}}^{\phi}=\frac{3}{2}\left\|\dot{\varepsilon}^{\mathrm{p} \phi}\right\| \frac{\mathrm{S}_{\mathrm{ij}}^{\phi}}{\left\|\mathrm{S}^{\phi}\right\|}
$$

implies that the kinetics of Prandtl (1924) and Reuss (1930) be described by

$$
\dot{\lambda}^{\phi}=\frac{3\left\|\dot{\varepsilon}^{\mathrm{p} \phi}\right\|}{2\left\|\mathrm{~S}^{\phi}\right\|}
$$

where

$$
\left\|S^{\phi}\right\|=\sqrt{\frac{3}{2} S_{i j}^{\phi} S_{i j}^{\phi}} \quad \text { and } \quad\left\|\dot{\varepsilon}^{p \phi}\right\|=\sqrt{\frac{2}{3} \dot{\varepsilon}_{i j}^{\phi} \dot{\varepsilon}_{i j}^{\phi}}
$$

Equation (54) gives the von Mises criteria for stress and plastic strain rate when normalized for tension. The Prandtl-Reuss (Prandtl, 1924; Reuss, 1930) kinetic equation (53) can be expressed in terms of the local shear stresses, $\tau^{\phi}$, and plastic shear strain rates, $\dot{\gamma}^{\mathrm{p} \phi}$, acting on phase $\phi$ as

$$
\dot{\lambda}^{\phi}=\frac{3 \dot{\gamma}^{\mathrm{p} \phi}}{2 \mathrm{M}^{2} \tau^{\phi}}
$$


The Taylor factor, which has a value between 2.65 and 3.06 for cubic systems depending on the number of slip systems (Kocks, 1970), establishes the transformation

$$
\tau^{\phi}=\left\|\mathrm{S}^{\phi}\right\| / \mathrm{M} \quad \text { and } \quad \dot{\gamma}^{\mathrm{p} \phi}=\mathrm{M}\left\|\dot{\varepsilon}^{\mathrm{p} \phi}\right\|
$$

As shown in $\S 5.1, \dot{\gamma}^{\mathrm{p} \phi}$ is a function of $\tau^{\phi}, \mathrm{T}, \rho^{\phi}$, cell or subgrain size, $\mathrm{L}\left(=\mathrm{L}^{\mathrm{h}}+\mathrm{L}^{\mathrm{s}}\right)$ and $\mathrm{f}^{\mathrm{h}}$. Thus, equation (56) establishes a relationship between the Prandtl-Reuss (Prandtl, 1924; Reuss, 1930) kinetics and a set of microstructural variables, which naturally leads to a viscoplastic model based on dislocation physics.

Since the elastic and thermal moduli in the 'hard' and 'soft' regions are the same, the volume averaged stress, $\bar{\sigma}_{\mathrm{ij}}$, and strain, $\bar{\varepsilon}_{\mathrm{ij}}$, that one measures in a laboratory experiment on an isotropic Hookean material are described by

$$
\begin{gathered}
\bar{\sigma}_{k k}=\sigma_{k k}^{\phi}=3 \kappa\left(\bar{\varepsilon}_{k k}-\alpha_{c}\left(T-T_{0}\right) \delta_{k k}\right) \\
\bar{S}_{i j}=2 G\left(\bar{E}_{i j}-\bar{\varepsilon}_{\mathrm{ij}}^{p}\right)
\end{gathered}
$$

where $T_{0}$ is a reference temperature and $\bar{\varepsilon}_{\mathrm{ij}}^{\mathrm{p}}$ is the volume averaged plastic strain. It should be noted that $\sigma_{\mathrm{kk}}^{\phi}=\bar{\sigma}_{\mathrm{kk}}$ is a hydrostatic form of the Reuss (1929) composite approximation, and consequently, that $\varepsilon_{\mathrm{kk}}^{\phi}=\bar{\varepsilon}_{\mathrm{kk}}$ is a hydrostatic form of the Voigt (1889) composite approximation. Furthermore, since the Voigt and Reuss approximations are upper and lower bounds, respectively, it then follows that the hydrostatic response is exact. However, this is not the case for the deviatoric stress response. The self-consistent formulation that is employed here is a realistic approximation that lies between the extremes of the Voigt-Reuss bounds.

There are two long-range internal stress states that arise from this composite substructure; they are the backward, $\mathrm{B}_{\mathrm{ij}}$, and forward, $\mathrm{F}_{\mathrm{ij}}$ stress tensors, which were introduced in one-dimensional form in $\S 5$. The back stress is a derived property in DSV; whereas, it is a phenomenological variable in the classical theories of plasticity and viscoplasticity. It is defined as the difference between the averaged applied stress and the stress of the 'soft' region

$$
\begin{gathered}
B_{i j} \equiv \bar{\sigma}_{i j}-\sigma_{i j}^{s}=f^{h}\left(\sigma_{i j}^{h}-\sigma_{i j}^{s}\right) \\
B_{i j} \equiv \bar{S}_{i j}-S_{i j}^{s}=f^{h}\left(S_{i j}^{h}-S_{i j}^{s}\right)
\end{gathered}
$$

Similarly, the forward stress is defined as the difference between the stress in the 'hard' region and the averaged applied stress

$$
\begin{aligned}
& F_{i j} \equiv \sigma_{i j}^{h}-\bar{\sigma}_{i j}=\left(1-f^{h}\right)\left(\sigma_{i j}^{h}-\sigma_{i j}^{s}\right) \\
& F_{i j} \equiv S_{i j}^{h}-\bar{S}_{i j}=\left(1-f^{h}\right)\left(S_{i j}^{h}-S_{i j}^{s}\right)
\end{aligned}
$$

Therefore,

$$
B_{i j}=\frac{f^{h}}{1-f^{h}} F_{i j}
$$

which is the three-dimensional analog of equation (28). These long-range internal stresses are deviatoric as a consequence of the fact that the hydrostatic contributions for the stresses of the 'hard' and 'soft' regions are equivalent. As expected, the rule of mixtures (eq. (48)) is satisfied for the data shown in Fig. 33(a) since it is the requirement for equilibrium of the local stress fields. 
Compatibility between the strains in the 'hard' and 'soft' regions, as derived by the self-consistent method, produces the local strain fields (Freed et al., 1992)

$$
\begin{aligned}
& E_{i j}^{s}=\bar{E}_{i j}+\left(\frac{\beta}{1-\beta}\right) \frac{B_{i j}}{2 G} \\
& E_{i j}^{h}=\bar{E}_{i j}-\left(\frac{\beta}{1-\beta}\right) \frac{F_{i j}}{2 G}
\end{aligned}
$$

where $\beta=[2(4-5 v) / 15(1-v)] \approx 0.5$ is the shape factor for a spherical inclusion and $v$ is Poisson's ratio. Equations (62) and (63) also satisfy the rule of mixtures given by equation (48). Since the cells are assumed to be equiaxed, and therefore isotropic in their properties, it is reasonable to approximate them as spheres in the Eshelby analysis although in reality they are not spherical. Since cells are not spherical, it may be necessary to treat $\beta$ as a variable parameter rather than a constant as determined by the Eshelby analysis given above.

The last terms in equations (62) and (63) are the self-consistent correction to the Voigt (1889) approximation for strain compatibility, i.e., $\mathrm{E}_{\mathrm{ij}}^{\phi}=\overline{\mathrm{E}}_{\mathrm{ij}}$. These correction terms are a consequence of the Eshelby (1957) approximation for strain compatibility when implemented into the self-consistent framework of Budiansky and Wu (1962) as derived in Appendix A. The Voigt strain compatibility is achieved when $\beta=0$ (i.e. $v=0.8$ ) in equations (62) and (63). This compatibility condition has been used by several investigators (Mughrabi (1983, 1987); Nix et al., (1985(a), (b)); Vogler and Blum, 1990; Lan et al., 1992) to analyze the deformation behavior of 'hard' and 'soft' regions in the one-dimensional substructural model discussed in $\$ 5$ (Figs. 40 and 41). Equations (62) and (63) make apparent the additional contributions brought into the theory by Eshelby strain compatibility and, as a consequence, the importance of the role that the internal stresses play in assuring compatibility of the local strain fields. The order of magnitude for these corrections is that of the elastic strain, and therefore, they are second-order corrections in most inelastic applications. Nevertheless, they could significantly impact the overall response, for example, under non-proportional loading histories.

\subsection{Solution Algorithm for the DSV Model}

At the current time, $t$, all variables are assumed to be known. In other words, the set of global variables, $\bar{\sigma}_{i j}$, $\bar{\varepsilon}_{\mathrm{ij}}{ }^{-\mathrm{p}}$, ij and $\mathrm{T}$, and the set of local variables, $\sigma_{\mathrm{ij}}^{\mathrm{h}}, \sigma_{\mathrm{ij}}^{\mathrm{s}}, \varepsilon_{\mathrm{ij}}^{\mathrm{h}}, \varepsilon_{\mathrm{ij}}^{\mathrm{s}}, \varepsilon_{\mathrm{ij}}^{\mathrm{ph}}, \varepsilon_{\mathrm{ij}}^{\mathrm{ps}}, \rho^{\mathrm{h}}, \rho^{\mathrm{s}}, \mathrm{f}^{\mathrm{h}}$ and $\mathrm{L}$ have known values. At some future time, $t+\delta \mathrm{t}$, values are assigned to $\mathrm{T}$ and $\varepsilon_{\mathrm{ij}}$, while values for all remaining global and local variables are left to be calculated. The theory contains four microstructural or internal state variables: $\rho^{\mathrm{h}}, \rho^{\mathrm{s}}, \mathrm{f}^{\mathrm{h}}$ and $\mathrm{L}$. The algorithm presented below is the solution for this problem statement resulting from the theoretical construction given above.

The first-order, ordinary, differential equations describing DSV that must be solved are

$$
\begin{gathered}
\left(1+\frac{\beta \mathrm{f}^{\mathrm{h}}}{1-\beta}\right) \dot{\mathrm{S}}_{\mathrm{ij}}^{\mathrm{s}}+\left(2 \mathrm{G} \dot{\lambda}^{\mathrm{s}}+\frac{\beta}{1-\beta} \dot{\mathrm{f}}^{\mathrm{h}}-\frac{\dot{\mathrm{G}}}{\mathrm{G}}\right) \mathrm{S}_{\mathrm{ij}}^{\mathrm{s}}=2 \mathrm{G} \dot{\overline{\mathrm{E}}}_{\mathrm{ij}}+\frac{\beta}{1-\beta}\left(\mathrm{f}^{\mathrm{h}} \dot{\mathrm{S}}_{\mathrm{ij}}^{\mathrm{h}}+\dot{\mathrm{f}}^{\mathrm{h}} \mathrm{S}_{\mathrm{ij}}^{\mathrm{h}}\right) \\
\left(1+\frac{\beta\left(1-\mathrm{f}^{\mathrm{h}}\right)}{1-\beta}\right) \dot{S}_{\mathrm{ij}}^{\mathrm{h}}+\left(2 \mathrm{G} \dot{\lambda}^{\mathrm{h}}-\frac{\beta}{1-\beta} \dot{\mathrm{f}}^{\mathrm{h}}-\frac{\dot{\mathrm{G}}}{\mathrm{G}}\right) \mathrm{S}_{\mathrm{ij}}^{\mathrm{h}}=2 \mathrm{G} \dot{\bar{E}}_{\mathrm{ij}}+\frac{\beta}{1-\beta}\left(\left(1-\mathrm{f}^{\mathrm{h}}\right) \dot{\mathrm{S}}_{\mathrm{ij}}^{\mathrm{s}}-\dot{\mathrm{f}}^{\mathrm{h}} \mathrm{S}_{\mathrm{ij}}^{\mathrm{s}}\right) \\
\dot{\rho}^{\phi}=\mathrm{P}^{\phi}\left[\tau^{\varphi}, \rho^{\varphi}, \mathrm{f}^{\mathrm{h}}, \mathrm{L}, \mathrm{T}\right] \\
\dot{\mathrm{f}}^{\mathrm{h}}=\dot{\mathrm{F}}\left[\tau^{\varphi}, \rho^{\varphi}, \mathrm{f}^{\mathrm{h}}, \mathrm{L}, \mathrm{T}\right]
\end{gathered}
$$




$$
\dot{\mathrm{L}}=\dot{\mathrm{L}}\left[\tau^{\varphi}, \rho^{\varphi}, \mathrm{f}^{\mathrm{h}}, \mathrm{L}, \mathrm{T}\right]
$$

whose kinetics are described by

$$
\dot{\lambda}^{\phi}=\frac{3 \dot{\gamma}^{\mathrm{p} \phi}\left[\tau^{\varphi}, \rho^{\varphi}, \mathrm{f}^{\mathrm{h}}, \mathrm{L}, \mathrm{T}\right]}{2 \mathrm{M}^{2} \tau^{\varphi}}
$$

Equations (64) and (65) are obtained by combining the Prandtl-Reuss (Prandtl, 1924; Reuss, 1930) equation (50) with the time rate-of-change of the local deviatoric strains given in equations (62) and (63). Representative relationships describing $\dot{\gamma}^{\mathrm{p \phi}}$ and $\dot{\rho}^{\phi}$ for each phase are given by equations (29), (34) and (41) for the simple onedimensional substructure model (i.e., when $\beta=0$ ). The equations are likely to be more complex when the dislocation mechanics are formulated for the more realistic three-dimensional cell or subgrain model. This formulation has not been completed so that the present analysis is restricted to the one-dimensional case. The rate of change in the volume fraction of the 'hard' regions is detailed in $\$ 6.4$. The form of equation (68) describing the rate of change in the cell or subgrain size, $\dot{L}$, is poorly understood at present. This change in the subgrain size influences the creep rate through its influence on equations (64) to (66). The importance of including $\mathrm{L}$ comes from the fact that there is now considerable evidence to suggest that the subgrain size changes during primary creep (Fig. 18) (Hasegawa et al., 1971) and that it grows to a new equilibrium value after the stress is reduced from an initial creep stress (Eggeler and Blum, 1981; Ferreira and Stang, 1979, 1983; Goel et al., 1983; Soliman et al., 1983). However, as discussed in $\$ 4.0$, measurements of the subgrain size after a reduction in the creep stress have resulted in contradictory results. It is possible that the changes in the cell size with strain or time are much smaller than the subgrain size (Goel et al., 1983; Soliman et al., 1983). It is clear that equation (68) should lead to equation (18) when a steadystate is established after the stress drop. As discussed in $\$ 6.4$, the present analysis assumes that $L \propto 1 / \hat{\tau}$, where $\hat{\tau}$ is the average strength of the obstacles in the 'hard' and 'soft' regions.

After integrating the local deviatoric stresses and the internal state variables, one updates the hydrostatic response

$$
\bar{\sigma}_{k k}=3 \kappa\left(\bar{\varepsilon}_{\mathrm{kk}}-\alpha_{\mathrm{c}}\left(\mathrm{T}-\mathrm{T}_{0}\right) \delta_{\mathrm{kk}}\right)
$$

and the remaining global and local variables

$$
\begin{gathered}
\bar{S}_{i j}=\left(1-f^{h}\right) S_{i j}^{s}+f^{h} S_{i j}^{h} \\
\bar{\varepsilon}_{i j}^{p}=\bar{E}_{i j}-\frac{\bar{S}_{i j}}{2 G} \\
E_{i j}^{s}=\bar{E}_{i j}+\left(\frac{\beta}{1-\beta}\right) \frac{B_{i j}}{2 G} \\
E_{i j}^{h}=\bar{E}_{i j}-\left(\frac{\beta}{1-\beta}\right) \frac{F_{i j}}{2 G} \\
\varepsilon_{i j}^{p s}=\bar{\varepsilon}_{i j}^{p}+\left(\frac{1}{1-\beta}\right) \frac{B_{i j}}{2 G}
\end{gathered}
$$




$$
\varepsilon_{i j}^{p h}=\bar{\varepsilon}_{i j}^{p}-\left(\frac{1}{1-\beta}\right) \frac{F_{i j}}{2 G},
$$

whose derivations are given in the Appendix. As expected, equations (73) to (76) satisfy the rule of mixtures equation (48). It should be noted that $\mathrm{B}_{\mathrm{ij}}$ and $\mathrm{F}_{\mathrm{ij}}$ are related to $\mathrm{S}_{\mathrm{ij}}^{\mathrm{s}}$ and $\mathrm{S}_{\mathrm{ij}}^{\mathrm{h}}$ through equations (59b) and (60b).

The differential equations (64) and (65) for the evolution of the local stresses are strongly coupled and highly nonlinear; therefore, they are best solved using either semi-implicit or implicit integration methods. The nonlinearity is caused, for the most part, by the kinetics of $\lambda^{\phi}$. The right-hand side of these equations (i.e. the nonhomogeneous contribution) is where the coupling is strongest. In particular, a change in the stress in one region affects the change in stress of the other region, and vice versa. This coupling (in both the stress and stress rate) can lead to numerical instabilities for unreasonable initial guesses of the stress and stress rate in the two regions. For some integration algorithms, it may therefore be necessary to first implicitly integrate the uncoupled differential equations for the local stresses obtained by using the Voigt approximation

$$
\dot{\mathrm{S}}_{\mathrm{ij}}^{\phi}+\left(2 \mathrm{G} \dot{\lambda}^{\phi}-\dot{\mathrm{G}} / \mathrm{G}\right) \mathrm{S}_{\mathrm{ij}}^{\phi}=2 \mathrm{G} \dot{\bar{E}}_{\mathrm{ij}}
$$

to acquire a reasonable initial guess for $\mathrm{S}_{\mathrm{ij}}^{\phi}$ and $\dot{\mathrm{S}}_{\mathrm{ij}}^{\phi}$. These quantities should then be used to initiate an implicit integration of the more accurate differential equations (64) and (65) where, because of the coupling, the nonhomogeneous contributions of the right-hand sides are evaluated one iteration in arrears. Equation (77) is equivalent to setting the shape factor $\beta=0$ in equations (64) and (65). As stated earlier, the model is applied to the simpler case of $\beta=0$ corresponding to the substructure configuration described in $\$ 5.0$ and illustrated by Figs. 42 and 43 . The case $\beta=0$ is also examined using the one-dimensional dislocation model, although it is not strictly valid, in order to study the effect of $\beta$ on the stresses and strains developed within the material.

\subsection{Rate of Change of Microstructural Parameters}

Equations (64) to (68) require some knowledge of the rates of change of the microstructural parameters in order to evaluate the validity of the DSV model for situations where $\beta=0$. The rates of change in the dislocation densities are given by equations (34) and (41) for the one-dimensional case but these formulations are likely to change for a three-dimensional substructure model. The dislocation physics for the latter geometry is still to be formulated. As mentioned in $\S 6.3$, the functional form of equation (68) is unknown at present. Defining $\hat{\tau}$ as

$$
\hat{\tau}=f^{h} \hat{\tau}^{h}+\left(1-f^{h}\right) \hat{\tau}^{s}
$$

L can be expressed as a function of $\hat{\tau}$ through

$$
\mathrm{L}=\left(\mathrm{K} / \mathrm{M}^{\mathrm{m}}\right) \mathrm{b}(\mathrm{G} / \hat{\tau})^{\mathrm{m}}
$$

Thus,

$$
\dot{\mathrm{L}}=-\left(\mathrm{mK} / \mathrm{M}^{\mathrm{m}}\right) \mathrm{b}(\mathrm{G} / \hat{\tau})^{(\mathrm{m}+1)}(\dot{\hat{\tau}} / \mathrm{G})
$$

Equations (18a) and (b) represent the steady-state forms of equation (79) when $\hat{\tau}$ is proportional to the applied stress. In defining $\mathrm{L}$ in terms of $\hat{\tau}$, it is clear that $\mathrm{L}$ will change in a natural way after a stress change due to corresponding changes in $\mathrm{f}^{\mathrm{h}}, \hat{\tau}^{\mathrm{h}}, \hat{\tau}^{\mathrm{S}}$ and $\hat{\tau}$. 
The experimental data discussed in $\$ 3.0$ can be used to formulate an empirical relation for $\mathrm{f}^{\mathrm{h}}$ in terms of strain.

Noting that

it is found that

$$
\mathrm{L}=\mathrm{L}^{\mathrm{h}}+\mathrm{L}^{\mathrm{S}}
$$

$$
\mathrm{L}^{\mathrm{S}}=\mathrm{L}\left[\frac{1}{1+\chi}\right] \quad \text { and } \quad \mathrm{L}^{\mathrm{h}}=\mathrm{L}\left[\frac{\chi}{1+\chi}\right]
$$

Thus,

$$
\mathrm{f}^{\mathrm{h}}=1-\left[\frac{1}{1+\chi}\right]^{3}
$$

and

$$
\dot{\mathrm{f}}^{\mathrm{h}}=3\left[\frac{1}{1+\chi}\right]^{4} \dot{\chi}
$$

Using equations (22b), (22c), (83) and (84), both $\mathrm{f}^{\mathrm{h}}$ and $\mathrm{f}^{\mathrm{h}}$ can be obtained as a function of strain.

The parameters $f^{h}$ and $\chi$ can be used interchangeably as the independent variable. Equation (83) is similar in form to that derived by Dobes and Orlová (1990) but differs from that used by other investigators (Mughrabi, 1981, 1983, 1987; Nix et al., 1985(a) and (b), and Qian and Fan, 1991) who defined $\mathrm{f}^{\mathrm{h}}$ as a linear fraction and assumed that the latter was equal to the volume fraction.

\subsection{Coupling of DSV and Dislocation Physics}

One of the objectives of this paper outlined in $\S 1.2$ was to develop the theoretical basis for interlinking dislocation mechanisms occurring at the level of the substructure with the macroscopic stress state. The scale-up from the local to the global state is achieved through DSV (Fig. 2). The treatment presented in $\$ 5.1,6.2$ to 6.4 demonstrates that this coupling is fairly complex even for the relatively simple one-dimensional dislocation model. Equations (64) and (65), together with equations (69) to (76), form the basis for this interlinkage between the local and the global variables, provided that the functional forms for equations (66) to (68), $\dot{\mathrm{f}}^{\mathrm{h}}$ and $\dot{\gamma}^{\mathrm{p} \phi}$ are known. The local strain rates are described by equation (29) for the 'hard' and 'soft' regions, where the evolution of $\hat{\tau}^{\phi}$ during deformation can be obtained from equations (35) and (46). Equations (34) and (41) give the rate of change in the dislocation densities in the 'hard' and 'soft' phases for the one-dimensional dislocation model. These equations can be used along with equation (30) to evaluate the change in $\hat{\tau}^{\phi}$ with strain. The evolution of $\hat{\tau}^{\phi}$ during deformation also influences L through equation (80) so that the change in the cell or subgrain size can be evaluated in a natural manner. An examination of equations (64), (65) and (71) shows that their solution requires some knowledge of $\mathrm{f}^{\mathrm{h}}$ and $\dot{\mathrm{f}}^{\mathrm{h}}$, where the latter are related to $\chi$ and $\dot{\chi}$ through equations (83) and (84), respectively. Equations (22b) and (c) give the specific functional forms for $\dot{\chi}$ and $\chi$, respectively, based on the experimental data shown in figures 21 and 27. Since the global strain rate evolves with $\hat{\tau}^{\phi}$, the quantities $\chi$ and $\dot{\chi}$, and hence $\mathrm{f}^{\mathrm{h}}$ and $\dot{\mathrm{f}}^{\mathrm{h}}$, also evolve with $\hat{\tau}^{\phi}$. Equations (22b) and (c) are empirical representations for the evolution of $\chi$ and $\dot{\chi}$. In principle, the exact forms could be derived from theories dealing with dislocation patterning (Kubin, 1993) although their computations are likely to be quite involved.

\subsection{SUMMARY}

A detailed review of the current understanding of the effect of microstructural parameters on class $\mathrm{M}$ creep behavior is presented. Microstructural observations conducted in the power-law and exponential creep regimes sug- 
gest that creep is influenced by a complex interaction between several elements of the microstructure, such as dislocations, cells and subgrains, and steady-state behavior is attained when the microstructure reaches a dynamic equilibrium between these different substructural features. Thus, the formation of equiaxed subgrains and the observation of class $\mathrm{M}$ creep (i.e., power-law creep) need not be a sufficient condition for steady-state behavior. Instead, recent observations reveal that the refinement of cell boundaries and the formation of secondary subboundaries are equally important components of steady-state creep. Quantitative measurements of the ratio of the dimensions of the cell boundary to the cell interior suggest that steady-state behavior is likely when this ratio is about 0.2. It is demonstrated that the microstructures formed in the exponential creep region are similar to those formed during the early stages of normal primary creep in the class $\mathrm{M}$ region. These experimental observations are used to formulate a

phenomenological approach to understanding transient and steady-state deformation behavior in terms of the strain rate-stress deformation laws. A three-dimensional dislocation substructure creep model is developed and coupled with a modified one-dimensional dislocation model. Although the present dislocation analysis is still limited in scope, nevertheless, it extends previous analyses by taking into account the dynamic evolution of the microstructure during deformation.

\section{ACKNOWLEDGMENTS}

The authors thank Drs. R.M. Dickerson and M.V. Nathal for their comments and suggestions. The permission granted by various publishers and authors for reproducing some of the figures used in the manuscript is gratefully acknowledged. 


\section{REFERENCES}

Argon, A.S. and Moffatt, W.C. (1981). Climb of Extended Edge Dislocations. Acta Metall., 29, pp. 293-299.

Argon, A.S. and Takeuchi, S. (1981). Internal Stresses in Power-Law Creep. Acta Metall., 29, pp. 1877-1884.

Argon, A.S. and Haasen, P. (1993). A New Mechanism of Work Hardening in the Late Stages of Large Strain Plastic Flow in F.C.C. and Diamond Cubic Crystals. Acta Metall. Mater., 41, pp. 3289-3306.

Armstrong, P.J. and Frederick, C.O. (1966). A Mathematical Representation of the Multiaxial Bauschinger Effect. Report RD/B/N731. pp.1-16. Berkeley Nuclear Laboratories, Central Electric Generating Board, Berkeley, U.K.

Balasubramanian, N. and Li, J.C.M. (1970). The Activation Areas for Creep Deformation. J. Mater. Sci., 5, pp. 434-444.

Barr, L.W., Hoodless, I.M., Morrison, J.A. and Rudham, R. (1960). Effects of Grosss Imperfections on Chloride Ion Diffusion in Crystals of Sodium Chloride and Potassium Chloride. Trans. Farad. Soc., 56, pp. 697-708.

Barr, L.W., Morrison, J.A. and Schroeder, P.A. (1965). Anion Diffusion in Crystals of NaCl. J. Appl. Phys., 36, pp. 624-631.

Barrett, C.R. and Sherby, O.D. (1964). Steady-State Creep Characteristics of Polycrystalline Copper in the Temperature Range $400^{\circ}$ to $950^{\circ} \mathrm{C}$. Trans. TMS-AIME, 230, pp. 1322-1327.

Barrett, C.R. and Sherby, O.D. (1965). Influence of Stacking-Fault Energy on High-Temperature Creep of Pure Metals. Trans. AIME, 233, pp. 1116-1119.

Belkin, E.G., Demikhovskaya, N.N., Kurov, I.E. and Myshlyaev, M.M., Kinetics of Sub-Structure Formation During Creep in Aluminum. Phys. Stat. Sol., 16(a), pp. 425-431.

Bendersky, L. Rosen, A. and Mukherjee, A.K. (1985). Creep and Dislocation Substructure. Intern. Metals Review, 30, pp. 1-15.

Beniere, F., Beniere, M. and Chemla, M. (1970). Conductibilite, Nombres de Transport et Autodiffuison des Ions dans Differents Monocristaux de Chlorure de Sodium. J. Phys. Chem. Solids, 31, pp. 1205-1220.

Beniere, M., Beniere, F., Catlow, C.R.A., Shukla, A.K. and Rao, C.N.R. (1977). Energy of Migration of Monovalent Ions in NaCl: An Experimental and Theoretical Study. J. Phys. Chem. Solids, 38, pp. 521-527.

Biberger, M. and Blum, W. (1988). Subgrain Boundary Migration During Creep of LiF. In "Strength of Metals and Alloys (ICSMA 8)", (P.O. Kettunen, T.K. Lepistö and M.E. Lehtonen), vol. 2, pp. 875-880, Pergamon, Oxford.

Biberger, M. and Blum, W. (1992)(a). Subgrain Boundary Migration During Creep of LiF: I. Recombination of Subgrain Boundaries. Phil. Mag., 65 A, pp. 757-770.

Biberger, M. and Blum, W. (1992)(b). Subgrain Boundary Migration During Creep of LiF: II. Constant-Stress Experiments. Phil. Mag., 66 A, pp. 27-40.

Bird, J.E., Mukherjee, A.K. and Dorn, J.E. (1969). Correlations Between High-Temperature Creep Behavior and Structure. In "Quantitative Relation Between Properties and Microstructure" (D.G. Brandon and A. Rosen, eds.). Israel Universities Press, Jerusalem, pp. 255-342.

Blum, W and Ilschner B. (1967). Über das Kriechverhalten von NaCl-Einkristallen. Phys. Stat. Sol., 20, pp. 629-642. 
Blum, W. (1977). Dislocation Models of Plastic Deformation of Metals at Elevated Temperatures. Z. Metallkde, 68, pp. 484-492.

Blum, W., Absenger, A. and Feilhauer, R. (1980). Dislocation Structure in Polycrystalline AlZn During Transient and Steady State Creep. In "Strength of Metals and Alloys (ICSMA 5)", (P. Haasen, V. Gerold and G. Kostorz, eds.), vol. 1, pp. 265-270. Pergamon, Oxford.

Blum, W. and Finkel, A. (1982). New Technique for Evaluating Long Range Internal Back Stresses. Acta Metall., 30, pp. 1705-1715.

Blum, W., Straub, S. and Volger, S. (1991). Creep of Pure Materials and Alloys. In "Strength of Metals and Alloys (ICSMA 9)" (D.G. Brandon, R. Chaim and A. Rosen, eds.), vol. I, pp. 111-126. Freund, London.

Blum, W. (1993). High-Temperature Deformation and Creep of Crystalline Solids. In "Plastic Deformation and Fracture of Materials" (H. Mughrabi, Vol. ed.). Materials Science and Technology (R.W. Cahn, P. Haasen and E.J. Kramer, Series eds.), vol. 6, pp. 360-405. VCH Publishers, Weinheim, Germany.

Bonneville, J. and Escaig, B. (1979). Cross-Slipping Process and the Stress-Orientation Dependence in Pure Copper. Acta Metall., 27, pp. 1477-1486.

Budiansky, B. and Wu, T.T. (1962). Theoretical Prediction of Plastic Strains of Polycrystals. In "Proceedings of the Fourth U. S. National Congress of Applied Mechanics," vol. 2, pp. 1175-1185. American Society for Mechanical Engineers, New York.

Caillard, D. and Martin, J.L. (1987). New Trends in Creep Microstructural Models for Pure Metals. Rev. Phys. Appl., 22, pp. 169-183.

Carrard, M. and Martin, J.L. (1987). A Study of (001) Glide in [112] Aluminum Single Crystals I. Creep Characteristics. Phil. Mag., 56, pp. 391-405.

Carrard, M. and Martin, J.L. (1988). A Study of (001) Glide in [112] Aluminum Single Crystals II. Microscopic Mechanism. Phil. Mag., 58, pp. 491-505.

Carter, N.L. and Hansen, F.D. (1983). Creep of Rocksalt. Tectonophysics, 92, pp. 275-333.

Challenger, K.D. and Moteff, J. (1973). Quantitative Characterization of the Substructure of AISI 316 Stainless Steel Resulting from Creep. Metall. Trans., 4, pp. 749-755.

Clauer, A.H., Wilcox, B.A. and Hirth, J.P. (1970). Dislocation Substructure Induced by Creep in Molybdenum Single Crystals. Acta Metall., 18, pp. 381-397.

Coble, R.L. (1963). A Model for Boundary-Diffusion Controlled Creep in Polycrystalline Materials. J. Appl. Phys., 34, pp. 1679-1682.

Conrad, H. (1964). Thermally Activated Deformation of Metals. J. Metals, 16, pp. 582-588.

Davies, C.K.L., Davies, P.W. and Wilshire, B. (1965). The Effect of Variations in Stacking-Fault Energy on the Creep of Nickel-Cobalt Alloys. Phil. Mag. 12, pp. 827-839.

Davies, P.W., Nelmes, G., Williams, K.R. and Wilshire, B. (1973). Stress-Change Experiments During HighTemperature Creep of Copper, Iron, and Zinc. Metal Sci. J., 7, pp. 87-92.

Dobes, F. and Orlová, A. (1990). Estimation of the Volume Fraction of Hard and Soft Regions Formed During High temperatue Creep. Mater. Sci. Eng., 125 A, L5-L9. 
Dorn, J.E. and Jaffe, N. (1961). Effect of Temperature on the Creep of Polycrystalline Aluminum by the Cross-Slip Mechanism. Trans. AIME, 221, pp. 229-233.

Downing, H.L. and Friauf, R.J. (1970). Self-Diffusion of Na in NaCl. J. Phys. Chem. Solids, 31, pp. 845-847.

Duesbery, M.S., Louat, N.P. and Sadananda, K. (1992). The Mechanics and Energetics of Cross-Slip. Acta Metall. Mater., 40, pp. 149-158.

Eggeler, G. and Blum, W. (1981). Coarsening of the Dislocation Structure after Stress Reduction during Creep of $\mathrm{NaCl}$ Single Crystals. Phil. Mag., 44 A, pp. 1065-1084.

Escaig, B. (1968)(a). Sur le Glissement Dévié des Dislocations dans la Structure Cubique a Faces Centrées. J. Phys., 29, pp. 225-239.

Escaig, B. (1968)(b). Cross-Slipping Process in the F.C.C. Structure. In "Dislocation Dynamics," (A.R. Rosenfield, G.T. Hahn, A.L. Bement and R.I. Jaffee), McGraw-Hill, New York, pp. 655-677.

Eshelby, J.D. (1957). The Determination of the Elastic Field of an Ellipsoidal Inclusion, and Related Problems. Proc. Roy. Soc. (Lond.), 241 A, pp. 376-396.

Estrin, Y. and Mecking, H. (1984). A Unified Phenomenological Description of Work Hardening and Creep Based on One-Parameter Models. Acta Metall., 32, pp. 57-70.

Evans, A.G. and Rawlings, R.D. (1969). The Thermally Activated Deformation of Crystalline Materials. Phys. Stat. Sol., 34, pp. 9-31.

Exell, S. F. and Warrington, D. H. (1972). Sub-grain Boundary Migration in Aluminum. Phil Mag., 26, pp. 1121-1136.

Feltham, P. and Sinclair, R.A. (1962-63). Dislocation Configurations in Copper After High-Temperature Creep. J. Inst. Metals, 91, pp. 235-238.

Feltham, P. and Sinclair, R. (1972). High-Temperature Creep of Copper. Acta Metall., 20, pp. 1095-1099.

Ferreira, I. and Stang, R.G. (1979). Effect of Stress Reductions on the Creep Behavior and Subgrain Size in Aluminum Deformed at 573 K. Mater. Sci. Eng., 38, pp. 169-174.

Ferreira, I. and Stang, R.G. (1983). The Effect of Stress and Subgrain Size on the Creep Behavior of High Purity Aluminum. Acta Metall., 31, pp. 585-590.

Freed, A.D., Raj, S.V. and Walker, K.P. (1992). Three-Dimensional Deformation Analysis of Two-Phase Dislocation Substructures. Scripta Metall. Mater., 27, pp. 233-238.

Friedel, J. (1959). Dislocation Interactions and Internal Strains. In "Internal Stresses and Fatigue in Metals," (G.M. Rassweiler and W.L. Grube, eds.), Elsevier, Amsterdam. pp. 220-262.

Friedel, J. (1964). Dislocations, Pergamon, Oxford. pp. 161-165.

Friedel, J. (1977). Sur le Fluage par Déviation. Rev. Phys., 12, pp. 1649-1654.

Frost, H.J. and Ashby, M.F. (1975). Deformation-Mechanism Maps and the Creep of Tungsten Lamp Filaments. In "Rate Processes in Plastic Deformation of Materials", (J.C.M. Li and A.K. Mukherjee, eds.), American Society for Metals, Metals Park, OH. pp. 70-101. 
Frost, H.J. and Ashby, M.F. (1977). Deformation-Mechanism Maps for Pure Iron, Two Austenitic Stainless Steels, and a Low-Alloy Ferritic Steel. In "Fundamental Aspects of Structural Alloy Design”, (R.I. Jaffee and B.A. Wilcox, eds.), Plenum, New York. pp. 27-65.

Frost, H.J. and Ashby, M.F. (1982). Deformation-Mechanism Maps: The Plasticity and Creep of Metals and Ceramics, Pergamon, Oxford.

Garofalo, F. (1965). Fundamentals of Creep and Creep Rupture in Metals, Macmillan, New York.

Gibeling, J.C. and Nix, W.D. (1977). The Existence of a Friction Stress for High-Temperature Creep. Metal Sci., 11, pp. $453-458$.

Gibeling, J.C. and Nix, W.D. (1980). The Description of Elevated Temperature Deformation in Terms of Threshold Stresses and Back Stresses: A Review. Mater. Sci. Eng., 45, pp. 123-135.

Gibeling, J.C. and Nix, W.D. (1981). Observation of Anelastic Backflow Following Stress Reductions During Creep of Pure Metals. Acta Metall., 29, pp. 1769-1784.

Ginter, T.J., Soliman, M.S. and Mohamed, F.A. (1984). Effect of Creep Substructure on the Stress Exponent of Al Following Stress Reductions. Phil. Mag., 50 A, pp. 9-24.

Gittus, J. (1975). Creep, Viscoelasticity and Creep Fracture in Solids, John Wiley, New York.

Goel, A., Ginter, T.J. and Mohamed, F.A. (1983). Effect of Stress Reductions on the Stress Exponent and Subgrain Size in an Al-Zn Alloy. Metall. Trans., 14 A, pp. 2309-2318.

Gramoll, K.C., Walker, K.P. and Freed, A.D. (1991). Investigation of Effective Material Properties for Viscoplastic Fibrous Composites Using the Self-Consistent and Mori-Tanaka Methods. In "High Temperature Constitutive Modeling_Theory and Application”, (A.D. Freed and K.P. Walker, eds), MD-vol. 26/AMD-vol. 121, pp. 439-449, American Society of Mechanical Engineers, New York.

Gupta, V.P. and Strutt, P.R. (1967). Dislocation Structures in Copper Single Crystals Deformed in High Temperature Creep. Canad. J. Phys., 45, pp. 1213-1220.

Haasen, P., Messerschmidt, U. and Skrotzki, W. (1986). Low Energy Dislocation Structures in Ionic Crystals and Semiconductors. Mater. Sci. Eng., 81, pp. 493-507.

Hansen, N. and Jensen, D.J. (1991). Mechanisms of Deformation, Recovery and Recrystallization of Aluminum. In "Hot Deformation of Aluminum Alloys," (T.G. Langdon, H.D. Merchant, J.G. Morris and M.A. Zaidi), The Minerals, Metals and Materials Society, pp. 3-19.

Harper, J. and Dorn, J.E. (1957). Acta Metall., 5, pp. 654-665.

Harper, J.G., Shepard, L.A. and Dorn, J.E. (1958). Acta Metall., 6, pp. 509-518.

Harrison, L.G., Morrison, J.A. and Rudham, R. (1958). Chloride Ion Diffuison in Sodium Chloride. Trans. Farad. Soc., 106, pp. 106-115.

Hart, E.W. (1957). On the Role of Dislocations in Bulk Diffusion. Acta Metall., 5, pp. 597.

Hasegawa, T., Hasegawa, R. and Karashima, S. (1970). Etch-Pit Studies of the Dislocation Structures Developed During Creep Deformation of Copper Single Crystals. Trans. Jap. Inst. Metals, 11, pp. 101-106. 
Hasegawa, T., Karashima, S. and Hasegawa, R. (1971). Substructure Formation and Nonuniformity in Strain During High Temperature Creep of Copper Single Crystals. Metall. Trans., 2, pp. 1449-1455.

Hasegawa, T., Ikeuchi, Y. and Karashima, S. (1972). Internal Stress and Dislocation Structure During Sigmoidal Transient Creep of a Copper-16 at.\% Aluminum Alloy. Metal Sci. J., 6, pp. 78-82.

Heard, H.C. (1972). Steady-state Flow in Polycrystalline Halite at Pressure of 2 Kilobars. In "Flow and Fracture of Rocks", Geophysical Monograph Series, vol. 16, (H.C. Heard, I.Y. Borg, N.L. Carter and C.B. Raleigh, eds.), American Geophysical Union, Washington, D.C. pp. 191-209.

Herring, C. (1950). Diffusional Viscosity of A Polycrystalline Solid. J. Appl. Phys., 21, pp. 437-445.

Hirth, J.P. and Nix, W.D. (1969). An Analysis of the Thermodynamics of Dislocation Glide. Phys. Stat. Sol., 35, pp. $177-188$.

Ho, Y.K. and Pratt, P. L. (1983). Dislocation Pipe Diffusion in Sodium Chloride Crystals. Radiation Effects, 75 , pp. 183-192.

Hofmann, U. and Blum, W. (1993). Modeling Transient Creep of Pure Materials After Changes in Stress and Temperature. In "Aspects of High Temperature Deformation and Fracture in Crystalline Materials", Y. Hosoi, H. Yoshinaga, H. Oikawa and K. Maruyama, eds.), The Japan Institute of Metals, Sendai, Japan. pp. 625-632.

Hüther, W. and Reppich, B. (1973). Dislocation Structure During Creep of MgO Single Crystals. Phil. Mag., 28, pp. 363-371.

Ilschner, B. and Reppich, B. (1963). Das Kriechen von NaCl-Einkristallen bei 500 bis $750^{\circ}$ C. Phys. Stat. Sol., 3, pp. 2093-2100.

Jaffe, N. and Dorn, J.E. (1962). Effect of Stress on the Creep Rate of High-Purity Aluminum in the Cross-Slip Region. Trans. AIME, 224, pp. 1167-1173.

Johnson, W.R., Barrett, C.R. and Nix, W.D. (1972). The High-Temperature Creep Behavior of Nickel-Rich Ni-W Solid Solutions. Metall. Trans., 3, pp. 963-969.

Kassner, M.E. and Oldani, J.J. (1988). High-Strain Steady-State Flow in Silver at Ambient and Near-Ambient Temperatures. Scripta Metall., 22, pp. 41-46.

Kassner, M.E. (1989). The Rate Dependence and Microstructure of High-Purity Silver Deformed to Large Strains Between 0.16 and $0.30 \mathrm{~T}_{\mathrm{m}}$. Metall. Trans., $20 \mathrm{~A}$, pp. 2001-2010.

Kestenbach, H.J., da Silveira, T.L. and Monteiro, S.N. (1976). On Subgrain Formation during Creep of 316 Stainless Steel. Metall. Trans., 7 A, pp. 155-158.

Kestenbach, H.J., Krause, W. and da Silveira, T.L. (1978). Creep of 316 Stainless Steel Under High Stresses. Acta Metall., 26, pp. 661-670.

Kocks, U.F. (1970). The Relation Between Polycrystal Deformation and Single-Crystal Deformation. Metall. Trans., 1, pp. 1121-1143.

Kocks, U.F., Argon, A.S. and Ashby, M.F. (1975). Thermodynamics and Kinetics of Slip. Prog. Mater. Sci., 19, pp. 1-291.

Kocks, U.F. (1987). Constitutive Behavior Based on Crystal Plasticity. In "Unified Constitutive Equations for Creep and Plasticity," (A.K. Miller, ed.), Elsevier, New York. pp. 1-88. 
Kong, Q.P. and Li, Y. (1993). Investigation of the Climb of Extended Dislocations during High-Temperature Creep. Phil. Mag., 68 A, pp. 113-119.

Kozyrskiy, G. Ya., Okrainets, P.N. and Pischak, V.K. (1972)(a). Dependence of the Structural Changes and Mechanical Properties of Metals with and F.C.C. Lattice on the Energy of Stacking Faults. Phys. Metals Metallogr., 33, pp. 173-178.

Kozyrskiy, G. Ya., Okrainets, P.N. and Pischak, V.K. (1972)(b). Creep Characteristics and Substructural Misalignment of Metals with an F.C.C. Lattice. Phys. Metals Metallogr., 34, pp. 151-157.

Kröner, E. (1961). Zur Plastischen Verformung ds Vielkristalls. Acta Metall., 9, pp. 155-161.

Kubin, L.P. (1993), Dislocation Patterning. In "Plastic Deformation and Fracture of Materials" (H. Mughrabi, Volume ed.). Materials Science and Technology (R.W. Cahn, P. Haasen and E.J. Kramer, Series eds.), vol. 6, pp. 137-190. VCH Publishers, Weinheim, Germany.

Lagneborg, R. (1972). Dislocation Mechanisms in Creep. Intern. Metall. Reviews, 17, pp. 130-146.

Lan, Y., Klaar, H.J. and Dahl, W. (1992). Evolution of Dislocation Structures and Deformation Behavior of Iron at Different Temperatures. Metall. Trans., 23 A, pp. 537-549.

Langdon, T.G. and Mohamed, F.A. (1977). The Characteristics of Independent and Sequential Processes. J. Austral. Inst. Metals, 22, pp. 189-199.

Langdon, T.G., Vastava, R.B. and Yavari, P. (1980). The Influence of Substructure on Creep Behavior Following Stress Changes. In "Strength of Metals and Alloys (ICSMA 5)", (P. Haasen, V. Gerold and G. Kostorz, eds.), vol. 1, pp. 271-276. Pergamon, Oxford.

Le Comte, P. (1965). Creep in Rock Salt. J. Geology, 73, pp. 469-481.

Lee, J.K and Nam, S.W. (1988). Effects of Cyclic Stress on the Creep Behavior and Dislocation Microstructure of Pure Copper in the Temperature Range 0.4 to $0.5 \mathrm{~T}_{\mathrm{m}}$. J. Mater. Sci., 23, pp. 2051-2058.

Longquan, S. and Northwood, D.O. (1993). Dislocation Network Models for Recovery Creep Deformation. J. Mater. Sci., 28, pp. 5963-5974.

Lüthy, H., Miller, A.K., Sherby, O.D.. (1980). The Stress and Temperature Dependence of Steady-State Flow at Intermediate Temperatures for Pure Polycrystalline Aluminum. Acta Metall., 28, pp. 169-178.

McLean, D. (1966). The Physics of High Temperature Creep in Metals. Reports Prog. Phys., 29, pp. 1-33.

Mecking, H. and Kocks, U.F. (1981). Kinetics of Flow and Strain-Hardening. Acta Metall., 29, pp. 1865-1875.

Michel, D.J., Moteff, J. and Lovell, A.J. (1973). Substructure of Type 316 Stainless Steel Deformed in Slow Tension at Temperatures Between $21^{\circ}$ and $816^{\circ} \mathrm{C}$. Acta Metall., 21, pp. 1269-1277.

Miller, A.K., Robinson, S.L. and Sherby, O.D. (1977). On the Importance of Subgrain Size to the Creep Rate. Phil. Mag., 36, pp. 757-760.

Mitchell, J.L. and Lazarus, D. (1975). Effect of Heterovalent Impurities Codiffusing with Monovalent Tracers in Ionic Crystals. Phys. Rev. B, 12, pp. 734-752.

Mohamed, F.A. and Langdon, T.G. (1974). The Transition from Dislocation Climb to Viscous Glide in Creep of Solid Solution Alloys. Acta Metall., 22, pp. 779-788. 
Mohamed, F.A. and Langdon, T.G. (1974). Method of Estimating Stacking-Fault Energies in Alkali Halide Crystals Using Creep Data. J. Appl. Phys., 45, pp. 1965-1967.

Mohamed, F.A., Soliman, M.S. and Mostafa, M.S. (1985). Effect of Stress Reduction Ratio on the Creep Behavior of Al. Phil. Mag., 51, pp. 559-574.

Mughrabi, H. and Essmann, U. (1980). Annihilation of Dislocations During Glide at Low Temperatures. In "Strength of Metals and Alloys (ICSMA 5)," (P. Haasen, V. Gerold and G. Kostorz, eds.), vol. 2, pp. 1101 1106. Pergamon, Oxford.

Mughrabi, H. (1980). Microscopic Mechanisms of Metal Fatigue. In "Strength of Metals and Alloys (ICSMA 5)," (P. Haasen, V. Gerold and G. Kostorz, eds.), vol. 3, pp. 1615-1638, Pergamon, Oxford.

Mughrabi, H. (1981). Cyclic Plasticity of Matrix and Persistent Slip Bands in Fatigued Metals. In "Continuum Models of Discrete Systems 4," (O. Brulin and R.K.T. Hsieh, eds.), North Holland, Amsterdam, The Netherlands. pp. 241-257.

Mughrabi, H. (1983). Dislocation Wall and Cell Structures and Long-Range Internal Stresses in Deformed Metal Crystals. Acta Metall., 31, pp. 1367-1379.

Mughrabi, H. (1987). The Long-Range Internal Stress Field in the Dislocation Wall Structure of Persistent Slip Bands. Phys. Stat. Sol., 104, pp. 107-120.

Mukherjee, A.K., Bird, J.E. and Dorn, J.E. (1969). Experimental Correlations for High-Temperature Creep. Trans. ASM, 62, pp. 155-179.

Myshlyaev, M.M. (1967). Change in the Block Structure of Aluminum During Creep. Sovt. Phys. Solid State, 9 , pp. 937-941.

Myshlyaev, M.M. (1981). Dislocation Creep. Ann. Rev. Mater. Sci., 11, pp. 31-50.

Nabarro, F.R.N. (1948). Deformation of Crystals by the Motion of Single Ions. Report of a Conference on Strength of Solids, The Physical Society, London, pp. 75-90.

Nadgornyi, E.M. and Strunk, H.P. (1987). Dislocation Structure of Plastically Deformed NaCl Polycrystals. Phys. Stat. Sol., 104(a), pp. 193-202.

Nadgornyi, E.M. (1988). Dislocation Dynamics and Mechanical Properties of Crystals, Prog. Mater. Sci., 31, pp. 1-536.

Nelson, V.C. and Friauf, R.J. (1970) Diffusion of Vacancies and Vacancy Pairs in NaCl. J. Phys. Chem. Solids, 31, pp. $825-843$.

Nix, W.D. and Ilschner, B. (1980). Mechanisms Controlling Creep of Single Phase Metals and Alloys. In "Strength of Metals and Alloys (ICSMA 5)", (P. Haasen, V. Gerold and G. Kostorz, eds.), vol. 3, pp. 1503-1530.

Pergamon, Oxford.

Nix, W.D. and Gibeling, J.C. (1985)(a). Mechanisms of Time-Dependent Flow and Fracture of Metals. In "Flow and Fracture at Elevated Temperatures" (R. Raj, ed.), American Society for Metals, Metals Park, OH, pp. 1-63.

Nix, W.D., Gibeling, J.C. and Hughes, D.A. (1985)(b). Time-Dependent Deformation of Metals. Metall. Trans., 16 A, pp. 2215-2226.

Oikawa, H. and Karashima, S. (1971). Effect of Stacking-Fault Energy on Steady-State Creep Rates in Copper-Base Solid Solutions. Scripta Metall., 5, pp. 909-914. 
Okrainets, P.N. and Pishchak, V.K. (1979). Stress Dependence of the Creep Rate for Metals with an F.C.C. Lattice. Phys. Metals Metallogr., 46, pp. 123-127.

Orlová, A., Pahutová, M. and Cadek, J. (1972)(a). Dislocation Structure and Applied, Effective and Internal Stress in High-Temperature Creep of Alpha Iron. Phil. Mag., 25, pp. 865-877.

Orlová, A., Tobolová, Z. and Cadek, J. (1972)(b). Internal Stress and Dislocation Structure of Aluminum in HighTemperature Creep. Phil. Mag., 26, pp. 1263-1274.

Orlová, A. and C̆adek, J. (1973). Some Substructural Aspects of High-Temperture Creep in Metals. Phil. Mag., 28, pp. 891-899.

Orlová, A. and Cadek, J. (1986). Dislocation Structure in the High Temperature Creep of Metals and Solid Solution Alloys: A Review. Mater. Sci. Eng., 77, pp. 1-18.

Pahutová, M., Čadek, J. and Ry š, P. (1977). Anelasticity and Measured Internal Stress in High Temperatue Creep. Scripta Metall., 11, pp. 1061-1066.

Pahutová, M., Dlouhy', A. and Čadek, J. (1993). An Experimental Method to Estimate Internal Stress Level During High Temperature Creep. Scripta Metall. Mater., 29, pp. 599-604.

Parker, J.D. and Wilshire, B. (1976). On the Subgrain Size Dependence of Creep. Phil. Mag., 34, pp. 485-489.

Pharr, G.M. (1981). Some Observations on the Relation Between Dislocation Substructure and Power Law Breakdown in Creep. Scripta Metall., 15, pp. 713-717.

Poirier, J.P. (1972). High-Temperature Creep of Single Crystalline Sodium Chloride I. Creep-controlling Mechanism. Phil. Mag., 26, pp. 701-712.

Poirier, J.P. (1972). High-Temperature Creep of Single Crystalline Sodium Chloride II. Investigation of the Creep Substructure. Phil. Mag., 26, pp. 713-725.

Poirier, J.P. (1976). On the Symmetrical Role of Cross-Slip of Screw Dislocations and Climb of Edge Dislocations as Recovery Processes Controlling High-Temperature Creep. Rev. Phys., 11, pp. 731-738.

Poirier, J.P. (1978). Is Power-Law Creep Diffusion-Controlled? Acta Metall., 26, pp. 629-637.

Poirier, J.P. (1979). Reply to “Diffusion-Controlled Dislocation Creep: A Defense”. Acta Metall., 27, pp. 401-403.

Poirier, J.P. (1985). Creep of Crystals: High-Temperature Deformation Processes in Metals, Ceramics and Minerals. Cambridge University Press, Cambridge, MA.

Pontikis, V. and Poirier, J. P. (1975). Phenomenological and Structural Analysis of Recovery-Controlled Creep, with Special Reference to the Creep of Single-Crystal Silver Chloride. Phil. Mag., 32, pp. 577-592.

Prandtl, L. (1924). Spannungsverteilung in Plastischen Körpern. In "Proceedings of the First International Congress on Applied Mechanics," (C.B. Biezeno and J.M. Burgers, eds.), Delft - Technische Boekhandel En Drukkerij J. Waltman, Jr., Delft, The Netherlands, pp. 43-54.

Prinz, F., Argon, A.S. and Moffatt, W.C. (1982). Recovery of Dislocation Structures in Plastically Deformed Copper and Nickel Single Crystals. Acta Metall., 30, pp. 821-830.

Przystupa, M.A., Lin, P., Ardell, A.J. and Ajaja, O. (1987). Dynamic Recovery During Compression Testing of Monocrystalline $\mathrm{NaCl}$ at Elevated Temperatures. Mater. Sci. Eng., 92, pp. 63-70. 
Püschl, W. and Schoeck, G. (1993). Calculation of Cross-Slip Parameters in F.C.C. Crystals. Mater. Sci. Eng., 164 B, pp. 286-289.

Qian, Z. and Fan, J. (1991). Constitutive Model for Creep-Plasticity Interaction Based on Continuous Kinetics of Dislocations. In "High Temperature Constitutive Modeling-Theory and Application", (A.D. Freed and K.P. Walker, eds), MD-vol. 26/AMD-vol. 121, pp. 49-63, American Society of Mechanical Engineers, New York.

Raj, R. and Ashby, M. F. (1971). On Grain Boundary Sliding and Diffusional Creep. Metall. Trans., 2, pp. 1113-1127.

Raj, S.V. (1986). The Effect of Stacking Fault Energy on the Creep Power-Law Breakdown Criterion in FCC Metals. Scripta Metall., 20, pp. 1333-1338.

Raj, S.V. and Pharr, G.M. (1986)(a). A Compilation and Analysis of Data for the Stress Dependence of the Subgrain Size. Mater. Sci. Eng., 81, pp. 217-237.

Raj, S.V. and Pharr, G.M. (1986)(b). Unpublished research. Rice University, Houston, TX.

Raj, S.V. (1989). Apparent Activation Volume for Creep of Copper and Alpha Brass at Intermediate Temperatures. J. Mater. Sci., 24, pp. 3196-3204.

Raj, S.V. and Langdon, T.G. (1989). Creep Behavior of Copper at Intermediate Temperatures-I. Mechanical Characteristics. Acta Metall., 37, pp. 843-852.

Raj, S.V., Pharr, G.M., and Whittenberger, J.D. (1989). On the Stability of the Creep Substructure in NaCl Single Crystals. Mater. Sci. Eng., A 113, pp. 161-175.

Raj, S.V. and Pharr, G.M. (1989). Creep Substructure Formation in Sodium Chloride Single Crystals in the Power Law and Exponential Creep Regimes. Mater. Sci. Eng., A 122, pp. 233-242.

Raj, S.V. and Langdon, T.G. (1991)(a). Creep Behavior of Copper at Intermediate Temperatures-II. Surface Microstructural Observations. Acta Metall., 39, pp. 1817-1822.

Raj, S.V. and Langdon, T.G. (1991)(b). Creep Behavior of Copper at Intermediate Temperatures-III. A Comparison with Theory. Acta Metall., 39, pp. 1823-1832.

Raj, S.V., Whittenberger, J.D. and Pharr, G.M. (1991). Comments on the Paper "Does the Subgrain Size Influence the Rate of Creep". Mater. Sci. Eng., A 145, L13-L17.

Raj, S.V. and Freed, A.D. (1992). Effect of Strain and Stress on the Relative Dimensions of the 'Hard' and 'Soft' Regions in Crept $\mathrm{NaCl}$ Single Crystals. Scripta Metall. Mater., 27, pp. 1741-1746.

Raj, S.V. and Pharr, G.M. (1992). Effect of Temperature on the Formation of Creep Substructure in Sodium Chloride Single Crystals. Amer. Ceram. Soc., 75, pp. 347-352.

Raj, S.V. (1994). Creep and Fracture of Dispersion-Strengthened Materials. In "Mechanical Properties of Metallic Composites" (S. Ochiai, ed.), Marcel Dekker, New York pp. 97-183.

Reuss, A. (1929). Berechnung der Fliessgrenze von Mischkristallen auf Grund der Plastizitätsbedingung für Einkristalle. Zeit. Angewand. Math. Mech., 9, pp. 49-58.

Reuss, A. (1930). Berücksichtigung der Elastischen Formänderung in der Plastizitätstheorie. Zeit. Angewand. Mat. Mech., 10, pp. 266-274. 
Robinson, S.L. and Sherby, O.D. (1969). Mechanical Behavior of Polycrystalline Tungsten at Elevated Temperature. Acta Metall., 17, pp. 109-125.

Robinson, S.L., Burke, P.M. and Sherby, O.D. (1974). Activation Energy and Subgrain Size-Creep Rate Relations in Sodium Chloride. Phil. Mag., 29, pp. 423-427.

Rothman, S.J., Peterson, N.L., Laskar, A.L. and Robinson, L.C. (1972). J. Phys. Chem. Solids, 33, pp. $1061-1069$.

Schoeck, G. (1957). Theory of Creep. Creep and Recovery, American Society for Metals, Metals Park, OH, pp. 199-226.

Schoeck, G. and Seeger, A. (1955). Activation Energy Problems Associated with Extended Dislocations. In "Report of the Conference on Defect in Crystalline Solids", The Physical Society, London. pp. 340-346.

Shalayev, V.I., Tkachenko, I.B. and Pavlov, V.A. (1969). Influence of Stacking Fault Energy on Creep of F.C.C. Metals. Phys. Metals Metallogr., 27, pp. 136-143.

Sherby, O.D. (1962). Factors Affecting the High Temperature Strength of Polycrystalline Solids. Acta Metall., 10, pp. $135-147$.

Sherby, O.D. and Burke, P.M. (1967). Mechanical Behavior of Crystalline Solids at Elevated Temperature. Prog. Mater. Sci., 13, pp. 325-390.

Sherby, O.D. and Young, C.M. (1975). Some Factors Influencing the Strain Rate-Temperature Dependence of the Flow Stress in Polycrystalline Solids. In "Rate Processes in Plastic Deformation of Materials", (J.C.M. Li and A.K. Mukherjee, eds.), American Society for Metals, Metals Park, OH. pp. 497-541.

Sherby, O.D., Klundt, R.H. and Miller, A.K. (1977). Flow Stress, Subgrain Size, and Subgrain Stability at Elevated Temperature. Metall. Trans., 8 A, pp. 843-850.

Sherby, O.D. and Weertman, J. (1979). Diffusion-Controlled Dislocation Creep: A Defense. Acta Metall., 27, pp. $387-400$.

Shewmon, P.G. (1963). Diffusion in Solids. McGraw-Hill, New York. pp. 175-178.

Singh Deo, N.N. and Barrett, C.R. (1969). High-Temperature Creep of Some Dilute Copper Silicon Alloys. Trans. AIME, 245, pp. 2467-2473.

Skrotzki, W. and Haasen, P. (1988). The Role of Cross Slip in the Steady State Creep of Salt. In "Second Conf. on the Mechanical Behavior of Salt,” Trans Tech Publications, Clausthal-Zellerfeld, Germany. pp 69-81.

Soliman, M.S., Ginter, T.J. and Mohamed, F.A. (1983). An Investigation of the Stress Exponent and Subgrain Size in $\mathrm{Al}$ after Stress Reduction. Phil. Mag., 48 A, pp. 63-81.

Spingarn, J.R., Barnett, D.M. and Nix, W.D. (1979). Theoretical Descriptions of Climb Controlled Steady State Creep at High and Intermediate Temperatures. Acta Metall., 27, pp. 1549-1561.

Streb, G. and Reppich, B. (1973). Steady State Deformation and Dislocation Structure of Pure and Mg-Doped LiF Single Crystals. Phys. Stat. Sol., 16(a), pp. 493-505.

Stokes, R.J. (1966). Mechanical Properties of Polycrystalline Sodium Chloride. Proc. Brit. Ceram. Soc., 6, pp. 189-207. 
Takeuchi, S. and Argon, A.S. (1976). Steady-State Creep of Single-Phase Crystalline Matter at High Temperature. J. Mater. Sci., 11, pp. 1542-1566.

Taylor, G.I. (1934). The Mechanism of Plastic Deformation of Crystals. Proc. Roy. Soc. (Lond.), 145 A, pp. 362-387.

Taylor, G.I. (1938). Plastic Strain in Metals. J. Inst. Metals, 62, pp. 307-324.

Thompson, A.W. (1977). Substructure Strengthening Mechanisms. Metall. Trans., 8 A, pp. 833-842.

Toma, K., Yoshinaga, H. and Morozumi, S. (1976). Internal Stresses During High-Temperature Deformation of Pure Aluminum and an Al-Mg Alloy. Trans. Jap. Inst. Metals, 17, pp. 102-110. .

Vandervoort, R.R. (1969). On the Possible Influence of Stacking Fault Energy on the Creep of Pure BCC Metals. Trans. AIME, 245, pp. 2269-2075.

Vogler, S. and Blum, W. (1990). Micromechanical Modelling of Creep in Terms of the Composite Model. In "Creep and Fracture of Engineering Materials and Structures", (B. Wilshire and R.W. Evans, eds.), The Institute of Metals, London. pp. 65-79.

Vogler, S., Messner, A. and Blum, W. (1991). Does the Subgrain Size Influence the Rate of Creep? Mater. Sci. Eng., 145 A, pp. 13-19.

Voigt, W. (1889). Über die Beziehung zwischen den beiden Elatizitätskonstanten isotroper Körper. Wied. Ann., 38, pp. 573-589.

Vollersten, F., Hofbeck, R. and Blum, W. (1984). Double Etching-A Simple Method of Investigating Subboundary Migration During Creep. Mater. Sci. Eng., 67, pp. L9-L14.

von Mises, R. (1913). Mechanik der festen Körper im Plastisch-Deformablen Zustand. Nachriehten von der Königlichen Gesellschaft der Wissenschaften, Mathematisch-Physikalische Klasse, pp. 582-592.

Wang, J., Horita, H., Furukawa, M., Nemoto, M., Tsenev, N.K., Valiev, R.Z., Ma, Y. and Langdon, T.G. (1993). An Investigation of Ductility and Microstructural Evolution in an Al-3\% Mg Alloy with Submicron Grain Size. J. Mater. Res., 8, pp. 2810-2818.

Wawersik, W.R. (1984). Alternatives to a Class M Creep Model for Rock Salt at Temperatures Below $160^{\circ}$ C. In "Proc. 2nd. Conf. Mechanical Behavior of Salt," (H. R. Hardy, Jr. and M. Langer, eds.), Hannover, Germany. pp. 1-26.

Wawersik, W.R. (1985). Determination of Steady State Creep Rates and Activation Parameters for Rock Salt. In "Measurement of Rock Properties at Elevated Pressures and Temperatures", ASTM STP 869, American Society for Testing of Materials, Philadelphia, PA. pp. 72-92.

Weertman, J. (1955). Theory of Steady-State Creep Based on Dislocation Climb. J. Appl. Phys., 26, pp. 1213-1217.

Weertman, J. (1956). Creep of Polycrystalline Aluminum as Determined from Strain Rate Tests. J. Mech. Phys. Solids, 4, pp. 230-234.

Weertman, J. (1957). Steady-State Creep of Crystals. J. Appl. Phys., 10, pp. 1185-1189.

Weertman, J. (1965). Theory of the Influence of Stacking-Fault Width of Split Dislocations on High-Temperature Creep Rate. Trans. AIME, 233, pp. 2069-2075. 
Weertman. J. (1975). High Temperature Creep Produced by Dislocation Motion. In "Rate Processes in Plastic Deformation of Materials", (J.C.M. Li and A.K. Mukherjee, eds.), American Society for Metals, Metals Park, OH. pp. 315-336.

Wolf, H. (1960). Die Aktivierungsenergie für die Quergleitung aufgespaltener Schraubenversetzungen, Z. Naturforschung, 15 A, pp. 180-193.

Yamada, H. and Li, C.Y. (1975). Stress Relaxation and Mechanical Equation of State in Nickel and TD Nickel. In "Rate Processes in Plastic Deformation of Materials", (J.C.M. Li and A.K. Mukherjee, eds.), American Society for Metals, Metals Park, OH. pp. 298-314.

Yang, Z., Xiao, Y. and Shih, C. (1987). High Temperature Creep of Ni-Cr-Co Alloys and the Effect of Stacking Fault Energy. Z. Metallkde., 78, pp. 339-343.

Yavari, P. Mohamed, F.A. and Langdon, T.G. (1981). Creep and Substructure Formation in an Al-5\% Mg Solid Solution Alloy. Acta Metall., 29, pp. 1495-1507.

Yoshinaga, H. (1993). High-Temperature Deformation Mechanisms in Metals and Alloys. Mater. Trans., Jap. Inst. Metals, 34, pp. 635-645.

Young, C.M. and Sherby, O.D. (1973). Subgrain Formation and Subgrain-Boundary Strengthening in Iron-Based Materials. J. Iron Steel Inst., 211, pp. 640-647.

Zhu, Q. and Blum, W. (1993). Explanation of the Transition from Class M to Class A Deformation Behavior in Terms of the Composite Model. In "Aspects of High Temperature Deformation and Fracture in Crystalline Materials”, Y. Hosoi, H. Yoshinaga, H. Oikawa and K. Maruyama, eds.), The Japan Institute of Metals, Sendai, Japan. pp. 649-656. 


\section{APPENDIX}

\section{A Derivation of the Coupled Differential Equations Used in the DSV Model}

For a general anisotropic Hookean material, the averaged response is governed by

$$
\bar{\sigma}_{\mathrm{ij}}=\mathrm{D}_{\mathrm{ijk} \ell}\left(\bar{\varepsilon}_{\mathrm{k} \ell}-\bar{\varepsilon}_{\mathrm{k} \ell}^{\mathrm{p}}-\alpha_{\mathrm{k} \ell}\left(\mathrm{T}-\mathrm{T}_{0}\right)\right)
$$

while the local responses of the 'soft' and 'hard' regions are governed by

$$
\sigma_{\mathrm{ij}}^{\phi}=\mathrm{D}_{\mathrm{ijk} \ell}\left(\varepsilon_{\mathrm{k} \ell}^{\phi}-\varepsilon_{\mathrm{k} \ell}^{\mathrm{p} \phi}-\alpha_{\mathrm{k} \ell}\left(\mathrm{T}-\mathrm{T}_{0}\right)\right)
$$

where $\mathrm{D}_{\mathrm{ijkl}}$ is the elastic moduli tensor and $\alpha_{\mathrm{kl}}$ is the coefficient of thermal expansion tensor. They are assumed to be identical for the 'hard' and 'soft' regions, and for the averaged material. Similarly, T is assumed to be uniform over both the phases.

Taking into account the fact that the elastic and thermal moduli of both regions are the same, one obtains the following strain compatibility relation from the generalized self-consistent method (Gramoll et al., 1991)

$$
\varepsilon_{\mathrm{ij}}^{\phi}=\bar{\varepsilon}_{\mathrm{ij}}+\mathrm{S}_{\mathrm{ijk} \ell}\left(\varepsilon_{\mathrm{k} \ell}^{\mathrm{p} \phi}-\bar{\varepsilon}_{\mathrm{k} \ell}^{\mathrm{p}}\right)
$$

where $S_{\text {ijkl }}$ is the Eshelby tensor (Eshelby, 1957), whose components are constant valued for the family of ellipsoidal inclusions. Consequently, there is no transformation strain unless there is plastic strain. When substituted into equation (A2), this condition for strain compatibility can be written in terms of the stresses as follows

$$
\sigma_{\mathrm{ij}}^{\phi}=\bar{\sigma}_{\mathrm{ij}}-\mathrm{D}_{\mathrm{ijk} \ell}\left(\mathrm{I}_{\mathrm{k} \ell \mathrm{mn}}-\mathrm{S}_{\mathrm{k} \ell \mathrm{mn}}\right)\left(\varepsilon_{\mathrm{mn}}^{\mathrm{p} \phi}-\bar{\varepsilon}_{\mathrm{mn}}^{\mathrm{p}}\right)
$$

where $\mathrm{I}_{\mathrm{klmn}}$ is the identity tensor for second-rank symmetric tensors. This result is analogous to the one derived by Kröner (1961).

When the material is isotropic, the averaged stress-strain response described by equation (A1) becomes equations (57) and (58), while the local response given by equation (A2) leads to equations (49) and (50) when differentiated. In addition, the criterion for compatibility described by equation (A4) becomes

$$
\mathrm{S}_{\mathrm{ij}}^{\phi}=\overline{\mathrm{S}}_{\mathrm{ij}}-2 \mathrm{G}(1-\beta)\left(\varepsilon_{\mathrm{ij}}^{\mathrm{p} \phi}-\bar{\varepsilon}_{\mathrm{ij}}^{\mathrm{p}}\right)
$$

where, the fourth-rank tensors $\mathrm{D}_{\mathrm{ijkl}}, \mathrm{I}_{\mathrm{klmn}}$ and $\mathrm{S}_{\mathrm{klmn}}$ reduce to the scalars $2 \mathrm{G}, 1$ and $\beta$, respectively. Equation (A5) was first derived by Budiansky and Wu (1962) using another approach.

Solving for the deviatoric response, where the applied strain, $\overline{\mathrm{E}}_{\mathrm{ij}}$, is given and the local stresses, $\mathrm{S}_{\mathrm{ij}}^{\phi}$, are known from integration, six tensor equations with six tensor unknowns are obtained. They are the constitutive equations

$$
\begin{aligned}
& \bar{S}_{i j}=2 G\left(\bar{E}_{i j}-\bar{\varepsilon}_{i j}^{p}\right) \\
& S_{i j}^{s}=2 G\left(E_{i j}^{s}-\varepsilon_{i j}^{p s}\right) \\
& S_{i j}^{h}=2 G\left(E_{i j}^{h}-\varepsilon_{i j}^{p h}\right)
\end{aligned}
$$


the compatibility equations derived from equation (A5)

$$
S_{i j}^{S}+2 G(1-\beta) \varepsilon_{i j}^{p s}=S_{i j}^{h}+2 G(1-\beta) \varepsilon_{i j}^{p h}
$$

and the volume averages

$$
\begin{gathered}
\bar{S}_{i j}=\left(1-f^{h}\right) S_{i j}^{s}+f^{h} S_{i j}^{h}, \\
\bar{\varepsilon}_{i j}^{p}=\left(1-f^{h}\right) \varepsilon_{i j}^{p s}+f^{h} \varepsilon_{i j}^{p h},
\end{gathered}
$$

where $\left\{\bar{S}_{\mathrm{ij}}, \bar{\varepsilon}_{\mathrm{ij}}^{\mathrm{p}}, \mathrm{E}_{\mathrm{ij}}^{\mathrm{h}}, \mathrm{E}_{\mathrm{ij}}^{\mathrm{s}}, \varepsilon_{\mathrm{ij}}^{\mathrm{ph}}, \varepsilon_{\mathrm{ij}}^{\mathrm{ps}}\right\}$ are the set of unknowns. Inverting this linear system of six equations to solve for these six unknown variables in terms of the known variables $\left\{\bar{E}_{\mathrm{ij}}, \mathrm{S}_{\mathrm{ij}}^{\mathrm{h}}, \mathrm{S}_{\mathrm{ij}}^{\mathrm{s}}, \mathrm{f}^{\mathrm{h}}\right\}$ leads to equations (71) to (76). The fact that the coefficients of the tensors in equations (A6) to (A11) are all scalar quantities instead of fourth rank tensors greatly simplified the inversion of these equations. 
Public reporting burden for this collection of information is estimated to average 1 hour per response, including the time for reviewing instructions, searching existing data sources, gathering and maintaining the data needed, and completing and reviewing the collection of information. Send comments regarding this burden estimate or any other aspect of this collection of information, including suggestions for reducing this burden, to Washington Headquarters Services, Directorate for Information Operations and Reports, 1215 Jefferson Davis Highway, Suite 1204, Arlington, VA 22202-4302, and to the Office of Management and Budget, Paperwork Reduction Project (0704-0188), Washington, DC 20503.
1. AGENCY USE ONLY (Leave blank)
2. REPORT DATE
August 1995
3. REPORT TYPE AND DATES COVERED
Technical Memorandum

\section{TITLE AND SUBTITLE}

Modeling the Role of Dislocation Substructure During Class M and

Exponential Creep

6. $\operatorname{AUTHOR}(\mathrm{S})$

S.V. Raj, Ilana Seiden Iskovitz and A.D. Freed

WU-505-63-52

5. FUNDING NUMBERS

7. PERFORMING ORGANIZATION NAME(S) AND ADDRESS(ES)

8. PERFORMING ORGANIZATION REPORT NUMBER

National Aeronautics and Space Administration

Lewis Research Center

Cleveland, Ohio 44135-3191

E-8491

9. SPONSORING/MONITORING AGENCY NAME(S) AND ADDRESS(ES)

10. SPONSORING/MONITORING AGENCY REPORT NUMBER

National Aeronautics and Space Administration

Washington, D.C. 20546-0001

NASA TM-106986

\section{SUPPLEMENTARY NOTES}

S.V. Raj and A.D. Freed, NASA Lewis Research Center; Ilana Seiden Iskovitz, Ohio Aerospace Institute, NASA Lewis Research Center, 21000 Brookpark Road, Cleveland, Ohio 44135. Responsible person, S.V. Raj, organization code 5120, (216) 433-8195. Also published in "Unified Constitutive Laws of Plastic Deformation," editors A.S. Krausz and K. Krausz, pp. 343-439, Academic Press, San Diego, 1996.

12a. DISTRIBUTION/AVAILABILITY STATEMENT $12 \mathrm{~b}$. DISTRIBUTION CODE

Unclassified - Unlimited

Subject Categories 24 and 39

This publication is available from the NASA Center for Aerospace Information, (301) 621-0390.

13. ABSTRACT (Maximum 200 words)

The different substructures that form in the power-law and exponential creep regimes for single phase crystalline materials under various conditions of stress, temperature and strain are reviewed. The microstructure is correlated both qualitatively and quantitatively with power-law and exponential creep as well as with steady state and non-steady state deformation behavior. These observations suggest that creep is influenced by a complex interaction between several elements of the microstructure, such as dislocations, cells and subgrains. The stability of the creep substructure is examined in both of these creep regimes during stress and temperature change experiments. These observations are rationalized on the basis of a phenomenological model, where normal primary creep is interpreted as a series of constant structure exponential creep rate-stress relationships. The implications of this viewpoint on the magnitude of the stress exponent and steadystate behavior are discussed. A theory is developed to predict the macroscopic creep behavior of a single phase material using quantitative microstructural data. In this technique the thermally activated deformation mechanisms proposed by dislocation physics are interlinked with a previously developed multiphase, three-dimensional, dislocation substructure creep model. This procedure leads to several coupled differential equations interrelating macroscopic creep plasticity with microstructural evolution.

\section{SUBJECT TERMS}

Creep; Dislocation substructure; Subgrains; Cells; Power-law creep; Exponential creep

\begin{tabular}{|c|c|}
\hline 17. SECURITY CLASSIFICATION \\
$\begin{array}{c}\text { OF REPORT } \\
\text { Unclassified }\end{array}$ & $\begin{array}{c}\text { 18. SECURITY CLASSIFICATION } \\
\text { OF THIS PAGE } \\
\text { Unclassified }\end{array}$ \\
\hline
\end{tabular}
19. SECURITY CLASSIFICATION OF ABSTRACT Unclassified

15. NUMBER OF PAGES 79

16. PRICE CODE

A05

20. LIMITATION OF ABSTRACT 